\title{
Cyanomethylation of Substituted Fluorenes and Oxindoles with
}

Alkyl Nitriles

Gang Hong, Pradip D. Nahide, and Marisa C. Kozlowski*

Department of Chemistry, Roy and Diana Vagelos Laboratories, University of Pennsylvania, Philadelphia, Pennsylvania 19104, United States

\section{Supporting Information}

\section{Contents}

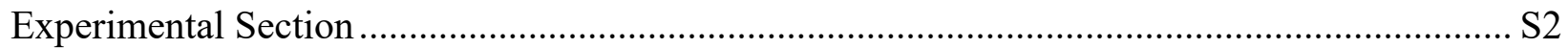

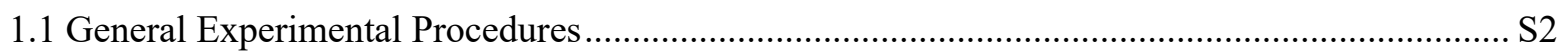

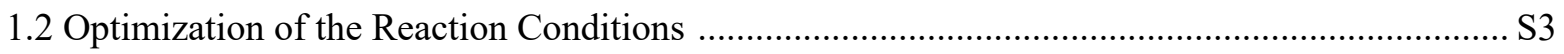

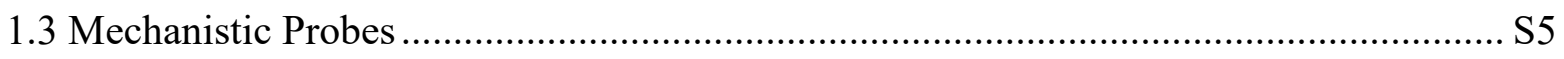

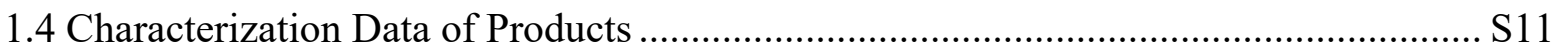

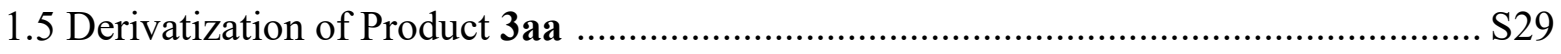

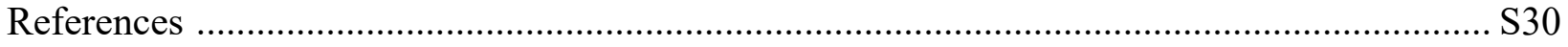

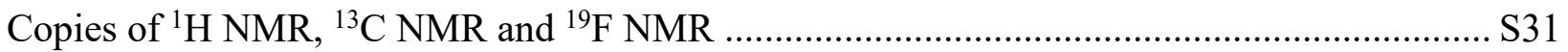




\section{Experimental Section}

Caution: Di-tert-butyl peroxide is a strong oxidizer and can be explosive. Exposure inhalation or skin contact is harmful. All experiments involving peroxy compounds should be carried out behind a safety shield and in a fume hood. ${ }^{1}$

All non-aqueous reactions were carried out under an atmosphere of dry argon unless otherwise noted. Commercial reagents were used as received without additional purification unless otherwise noted. 3-Substituted oxindoles used in this study were prepared according to the literature. ${ }^{2}$ 9Substituted fluorenes were prepared according to the literature. ${ }^{3}$ Reaction mixtures were heated in vials on thermostatted metal blocks using Ika plates. Reactions were monitored by thin layer chromatography (TLC) using Silicycle glass-backed TLC plates with $250 \mu \mathrm{m}$ silica and F254 indicator. Visualization was accomplished by UV light.

${ }^{1} \mathrm{H}$ NMR, ${ }^{13} \mathrm{C}$ NMR, and ${ }^{19} \mathrm{~F}$ NMR spectra were recorded on a AM-500 Fourier transform NMR spectrometer at $500 \mathrm{MHz}, 125 \mathrm{MHz}, 376 \mathrm{MHz}$ respectively. Chemical shifts are reported relative to the solvent resonance peak $\delta 7.26\left(\mathrm{CDCl}_{3}\right)$ for ${ }^{1} \mathrm{H}$ and $\delta 77.16\left(\mathrm{CDCl}_{3}\right)$ for ${ }^{13} \mathrm{C}$. Data are reported as follows: chemical shift, multiplicity $(\mathrm{s}=$ singlet, $\mathrm{d}=$ doublet, $\mathrm{t}=$ triplet, $\mathrm{q}=$ quartet, $\mathrm{b}$ = broad singlet, $\mathrm{m}=$ multiplet), coupling constants, and number of protons. High resolution mass spectra were obtained using a VG autospec with an ionization mode of EI or ESI-TOF. Infrared spectra are reported in $\mathrm{cm}^{-1}$. Column chromatography was performed with silica gel $(50-63 \mu \mathrm{m}$ mesh particle size).

\subsection{General Experimental Procedures}

General procedure A - cyanomethylation of 3-substituted oxindoles in acetonitrile. Oxindole $1(0.15 \mathrm{mmol})$ was added to a flame-dried $8 \mathrm{~mL}$ microwave vial equipped with stir bar and was 
brought into the glovebox. Acetonitrile $2 \mathrm{a}(0.10 \mathrm{M}, 1.5 \mathrm{~mL})$ and $t$-BuOOt-Bu $(110 \mu \mathrm{L}, 4.00$ equiv) were added to the mixture sequentially. The microwave vial was sealed with a Teflon cap, removed from the glovebox, and placed in a $130{ }^{\circ} \mathrm{C}$ oil bath. After $24 \mathrm{~h}$, the mixture was allowed to cool to ambient temperature and was concentrated. The residue was chromatographed (EtOAc/hexanes) to afford the product 3 .

\section{General procedure B - cyanomethylation of 9-substituted fluorenes in neat alkyl nitriles.}

Fluorene $5(0.15 \mathrm{mmol})$ was added to a flame-dried $8 \mathrm{~mL}$ microwave vial equipped with stir bar and was brought into the glovebox. PivOH ( $31 \mathrm{mg}, 0.30 \mathrm{mmol}, 2.0$ equiv), alkyl nitrile $2(0.10 \mathrm{M}$, $1.5 \mathrm{~mL})$ and $t$-BuOOt-Bu (165 $\mu \mathrm{L}, 6.00$ equiv) were added to the mixture sequentially. The microwave vial was sealed with a Teflon cap, removed from the glovebox, and placed in a $125^{\circ} \mathrm{C}$ oil bath. After $23 \mathrm{~h}$, the mixture was allowed to cool to ambient temperature and was concentrated. The residue was chromatographed (EtOAc/hexanes) to afford the desired product 6 .

\subsection{Optimization of the Reaction Conditions}

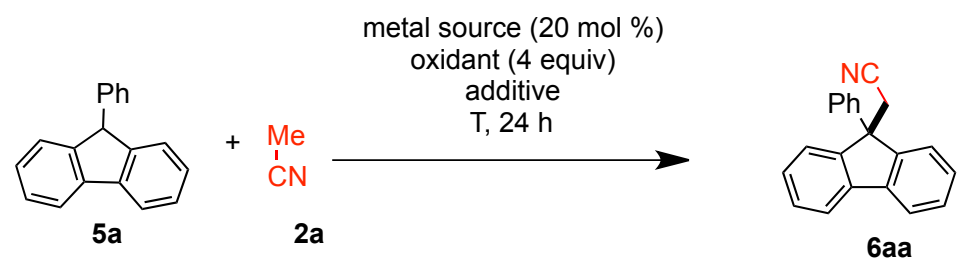

Table S1.

\begin{tabular}{|c|c|c|c|c|c|}
\hline Entry & Metal source & Oxidant $^{a}$ & Additive (equiv) & $\mathrm{T}\left({ }^{\circ} \mathrm{C}\right)$ & Yield $(\%)^{b}$ \\
\hline 1 & $\mathrm{CuBr}$ & $t$-BuOOt-Bu & Phen $(0.2)$ & 130 & 0 \\
\hline 2 & $\mathrm{Cu}(\mathrm{OTf})_{2}$ & $t$-BuOO $t-\mathrm{Bu}$ & Phen $(0.2) / \mathrm{Na}_{3} \mathrm{PO}_{4}(2)$ & 130 & 23 \\
\hline 3 & $\mathrm{CuI}$ & $t-\mathrm{BuOO} t-\mathrm{Bu}$ & Phen $(0.2)$ & 130 & 0 \\
\hline 4 & $\mathrm{Cu}(\mathrm{OAc})_{2}$ & $t-\mathrm{BuOO} t-\mathrm{Bu}$ & Phen (0.2)/PivOH (2) & 130 & 0 \\
\hline 5 & $\mathrm{CuCl}$ & $t-\mathrm{BuOO} t-\mathrm{Bu}$ & & 130 & 26 \\
\hline 6 & $\mathrm{CuCN}$ & $t$-BuOO $t-\mathrm{Bu}$ & & 130 & 46 \\
\hline 7 & $\mathrm{Pd}(\mathrm{OAc})_{2}$ & $t$-BuOO $t-\mathrm{Bu}$ & & 130 & 48 \\
\hline 8 & $\mathrm{Co}(\mathrm{OAc})_{2} \cdot 4 \mathrm{H}_{2} \mathrm{O}$ & $t$-BuOO $t-\mathrm{Bu}$ & & 130 & 36 \\
\hline 9 & $\mathrm{Mn}(\mathrm{OAc})_{3} \cdot 2 \mathrm{H}_{2} \mathrm{O}$ & $t-\mathrm{BuOO} t-\mathrm{Bu}$ & & 130 & 0 \\
\hline 10 & Ferrocene & $t-\mathrm{BuOO} t-\mathrm{Bu}$ & & 130 & 28 \\
\hline 11 & $\mathrm{FeCl}_{3}$ & $\mathrm{Ag}_{2} \mathrm{CO}_{3}(2)$ & & 130 & 13 \\
\hline
\end{tabular}




\begin{tabular}{|c|c|c|c|c|c|}
\hline 12 & $\mathrm{FeCl}_{3}$ & $t-\mathrm{BuOO} t-\mathrm{Bu}$ & $\mathrm{Ag}_{2} \mathrm{CO}_{3}(2)$ & 130 & $53^{c}$ \\
\hline 13 & $\mathrm{Sc}(\mathrm{OTf})_{3}$ & $\mathrm{Ag}_{2} \mathrm{O}(2)$ & & 130 & 15 \\
\hline 14 & & $t-\mathrm{BuOO} t-\mathrm{Bu}$ & $\mathrm{Ag}_{2} \mathrm{CO}_{3}(0.2)$ & 130 & $49^{c}$ \\
\hline 15 & & $t-\mathrm{BuOO} t-\mathrm{Bu}$ & & 130 & $54^{c}$ \\
\hline 16 & & DCP & & 130 & 9 \\
\hline 17 & & $\mathrm{BzOOBz}$ & & 130 & 46 \\
\hline 18 & & ТВРВ & & 130 & 25 \\
\hline 19 & & $t-\mathrm{BuOOH}$ & & 130 & 41 \\
\hline 20 & & $t-\mathrm{BuOO} t-\mathrm{Bu}$ & $\mathrm{KO}^{t} \mathrm{Bu}(1)$ & 130 & 0 \\
\hline 21 & & $t-\mathrm{BuOO} t-\mathrm{Bu}$ & $\mathrm{Cs}_{2} \mathrm{CO}_{3}(2)$ & 130 & 0 \\
\hline 22 & & $t-\mathrm{BuOO} t-\mathrm{Bu}$ & DBU (2) & 130 & trace \\
\hline 23 & & $t-\mathrm{BuOO} t-\mathrm{Bu}$ & PivOH (2) & 130 & $60^{c}$ \\
\hline 24 & & $t-\mathrm{BuOO} t-\mathrm{Bu}$ & HOAc (1) & 130 & 45 \\
\hline 25 & & $t-\mathrm{BuOO} t-\mathrm{Bu}$ & $\mathrm{TsOH}(2)$ & 130 & 11 \\
\hline 26 & & $t-\mathrm{BuOO} t-\mathrm{Bu}$ & $\mathrm{KI}(0.3)$ & 130 & 33 \\
\hline 27 & & $t-\mathrm{BuOO} t-\mathrm{Bu}$ & TBAI (0.2) & 130 & 48 \\
\hline 28 & & $t-\mathrm{BuOO} t-\mathrm{Bu}$ & PivOH (1) & 130 & $56^{c}$ \\
\hline 29 & & $t$-BuOOt-Bu (6) & PivOH (2) & 125 & $65^{c, d}$ \\
\hline 30 & & $t$-BuOOt-Bu (6) & $\mathrm{PivOH}(2)$ & 125 & $46^{e}(67)^{c, f}$ \\
\hline
\end{tabular}

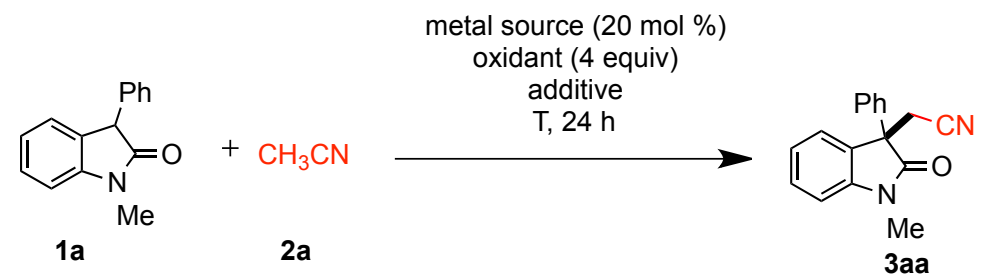

Table S2.

\begin{tabular}{|c|c|c|c|c|c|}
\hline Entry & Metal source & Oxidant $^{a}$ & Additive (equiv) & $\mathrm{T}\left({ }^{\circ} \mathrm{C}\right)$ & Yield $(\%)^{b}$ \\
\hline 1 & $\mathrm{Cu}(\mathrm{OTf})_{2}$ & $t-\mathrm{BuOO} t-\mathrm{Bu}$ & Phen (0.5)/ $\mathrm{Na}_{3} \mathrm{PO}_{4}(2)$ & 120 & 16 \\
\hline 2 & $\mathrm{Cu}(\mathrm{OTf})_{2}$ & $t-\mathrm{BuOO} t-\mathrm{Bu}$ & Phen $(0.5)$ & 120 & 20 \\
\hline 3 & $\mathrm{Cu}(\mathrm{OAc})_{2}$ & $t-\mathrm{BuOO} t-\mathrm{Bu}$ & & 120 & 16 \\
\hline 4 & $\mathrm{Cu}$ & $t-\mathrm{BuOO} t-\mathrm{Bu}$ & & 120 & 22 \\
\hline 5 & $\mathrm{Cu}_{2} \mathrm{O}$ & $t-\mathrm{BuOO} t-\mathrm{Bu}$ & & 120 & 17 \\
\hline 6 & $\mathrm{CuBr}(1)$ & $t-\mathrm{BuOO} t-\mathrm{Bu}$ & & 120 & 30 \\
\hline 7 & $\mathrm{CuCl}$ & $t-\mathrm{BuOO} t-\mathrm{Bu}$ & & 120 & 23 \\
\hline 8 & $\mathrm{FeCl}_{3}$ & $\mathrm{Ag}_{2} \mathrm{CO}_{3}$ & & 120 & $34^{b}$ \\
\hline 9 & $\mathrm{FeCl}_{3}$ & $t-\mathrm{BuOO} t-\mathrm{Bu}$ & & 120 & 12 \\
\hline 10 & $\mathrm{Fe}(\mathrm{acac})_{3}$ & $t-\mathrm{BuOO} t-\mathrm{Bu}$ & & 120 & trace \\
\hline 11 & Ferrocene & $t-\mathrm{BuOO} t-\mathrm{Bu}$ & & 120 & 33 \\
\hline 12 & $\mathrm{Sc}(\mathrm{OTf})_{3}$ & $\mathrm{Ag}_{2} \mathrm{O}(2)$ & & 120 & 30 \\
\hline 13 & $\mathrm{Sc}(\mathrm{OTf})_{3}$ & $t-\mathrm{BuOO} t-\mathrm{Bu}$ & & 120 & 12 \\
\hline
\end{tabular}




\begin{tabular}{|c|c|c|c|c|c|}
\hline 14 & $\mathrm{Zn}(\mathrm{OTf})_{2}$ & $t-\mathrm{BuOO} t-\mathrm{Bu}$ & & 120 & trace \\
\hline 15 & $\mathrm{Pd}(\mathrm{OAc})_{2}$ & $t-\mathrm{BuOO} t-\mathrm{Bu}$ & & 120 & $34^{b}$ \\
\hline 16 & $\mathrm{PdCl}_{2}$ & $t-\mathrm{BuOO} t-\mathrm{Bu}$ & & 120 & 22 \\
\hline 17 & $\mathrm{Pd}(\mathrm{OAc})_{2}$ & $\mathrm{~K}_{2} \mathrm{~S}_{2} \mathrm{O}_{8}$ & & 120 & 0 \\
\hline 18 & $\mathrm{CoCl}_{2} \cdot 6 \mathrm{H}_{2} \mathrm{O}$ & $t-\mathrm{BuOO} t-\mathrm{Bu}$ & & 120 & 27 \\
\hline 19 & $\mathrm{Ni}(\mathrm{OAc})_{2}$ & $t-\mathrm{BuOO} t-\mathrm{Bu}$ & & 120 & 8 \\
\hline 20 & $\mathrm{AlCl}_{3}$ & $t-\mathrm{BuOO} t-\mathrm{Bu}$ & & 120 & 0 \\
\hline 21 & & $t-\mathrm{BuOO} t-\mathrm{Bu}$ & & 120 & $40^{c}$ \\
\hline 22 & & ТВРB & & 120 & 38 \\
\hline 23 & & $t$-BuOOH & & 120 & trace \\
\hline 24 & & CHP & & 120 & 0 \\
\hline 25 & & $\mathrm{BzOOBz}$ & & 120 & 30 \\
\hline 26 & & DCP & & 120 & 38 \\
\hline 27 & & t-BuOOt-Bu & & 130 & $48^{c}$ \\
\hline 28 & & $t$-BuOOt-Bu & & 100 & 14 \\
\hline 29 & & $t$-BuOOt-Bu (2) & & 130 & 25 \\
\hline 30 & & $t$-BuOO $t$-Bu (6) & & 130 & 41 \\
\hline 31 & & $t-\mathrm{BuOO} t-\mathrm{Bu}$ & & 130 & $46^{c, d}$ \\
\hline 32 & & $t-\mathrm{BuOO} t-\mathrm{Bu}$ & & 130 & $35^{e}(47)^{f}$ \\
\hline 33 & & $t-\mathrm{BuOO} t-\mathrm{Bu}$ & KOH (1) & 130 & 0 \\
\hline 34 & & $t-\mathrm{BuOO} t-\mathrm{Bu}$ & $\mathrm{Na}_{3} \mathrm{PO}_{4}(2)$ & 130 & 9 \\
\hline 35 & & $t-\mathrm{BuOO} t-\mathrm{Bu}$ & DBU (1) & 130 & 0 \\
\hline 36 & & $t-\mathrm{BuOO} t-\mathrm{Bu}$ & $\mathrm{Cs}_{2} \mathrm{CO}_{3}(2)$ & 130 & 0 \\
\hline 37 & & $t-\mathrm{BuOO} t-\mathrm{Bu}$ & PivOH (2) & 130 & 29 \\
\hline 38 & & $t-\mathrm{BuOO} t-\mathrm{Bu}$ & $\mathrm{AcOH}(2)$ & 130 & 33 \\
\hline 39 & & $t-\mathrm{BuOO} t-\mathrm{Bu}$ & TsOH (2) & 130 & 0 \\
\hline 40 & & $t-\mathrm{BuOO} t-\mathrm{Bu}$ & TBAI (0.2) & 130 & 39 \\
\hline 41 & & $t-\mathrm{BuOO} t-\mathrm{Bu}$ & $\mathrm{I}_{2}(0.25)$ & 130 & 0 \\
\hline 42 & & $t-\mathrm{BuOO} t-\mathrm{Bu}$ & & 130 & $27^{g}$ \\
\hline
\end{tabular}

\subsection{Mechanistic Probes}

\section{Radical inhibitor/trap experiments.}




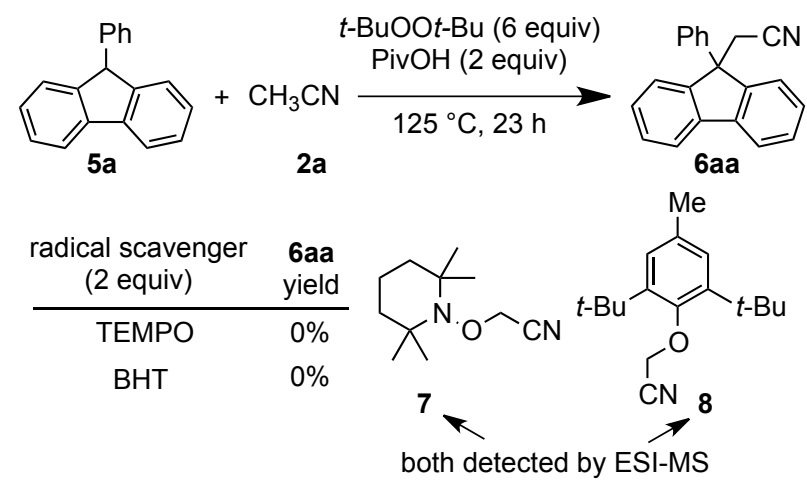

Compound 5a (36 mg, $0.15 \mathrm{mmol}$ ) was added to a flame-dried $8 \mathrm{~mL}$ microwave vial equipped with a stir bar and was brought into the glovebox. TEMPO (47 mg, 2.0 equiv), PivOH (31 mg, $0.30 \mathrm{mmol}, 2.0$ equiv), acetonitrile ( $0.10 \mathrm{M}, 1.5 \mathrm{~mL})$, and $t$-BuOOt-Bu (165 $\mu \mathrm{L}, 6.00$ equiv) were added to the mixture sequentially. The microwave vial was sealed with a Teflon cap, removed from the glovebox, and placed in a $125^{\circ} \mathrm{C}$ oil bath. After $23 \mathrm{~h}$, the mixture was allowed to cool to ambient temperature. Part of the mixture was analyzed by UPLC-MS, and the TEMPO-adduct was observed.

Figure S1.

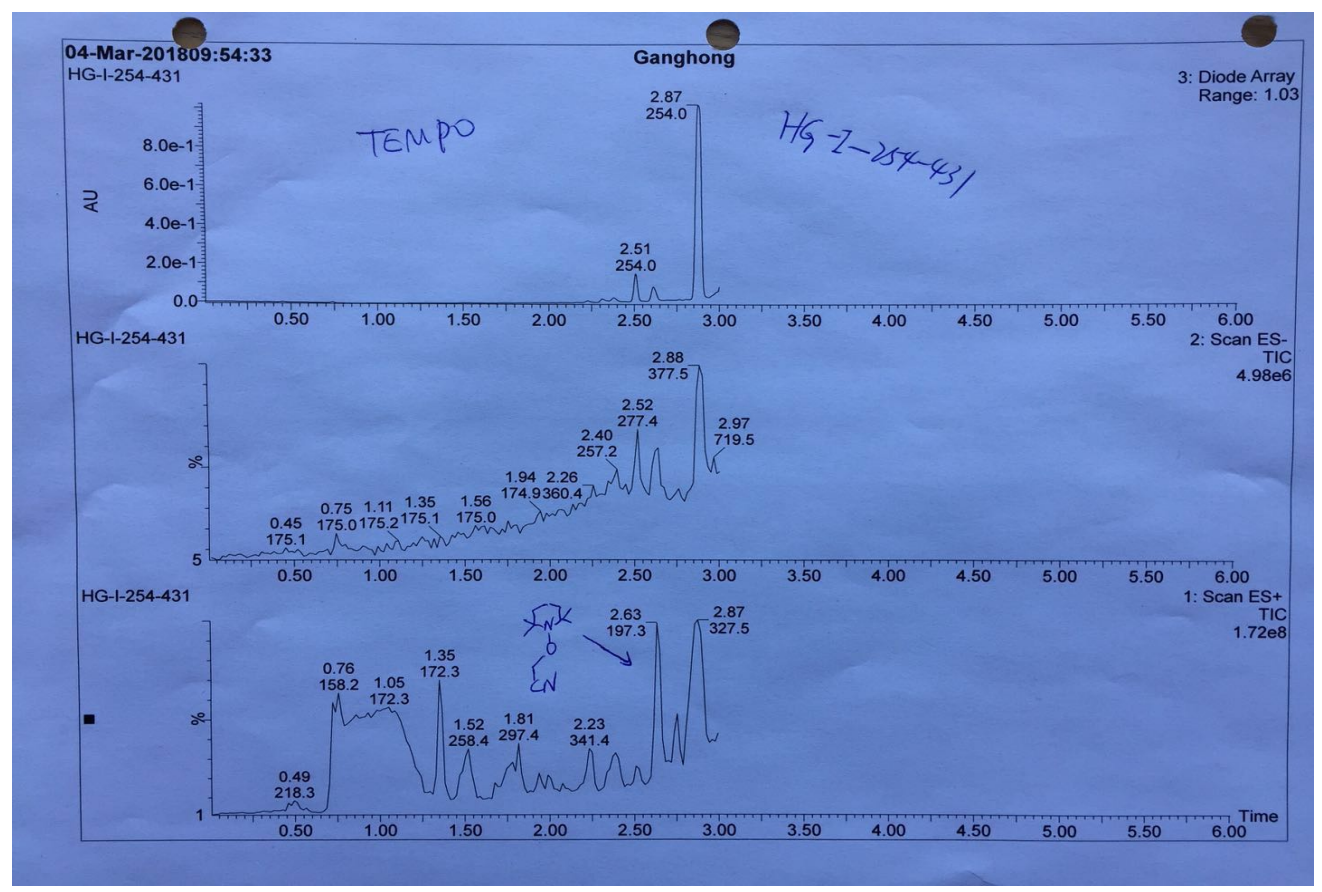


Figure S2.

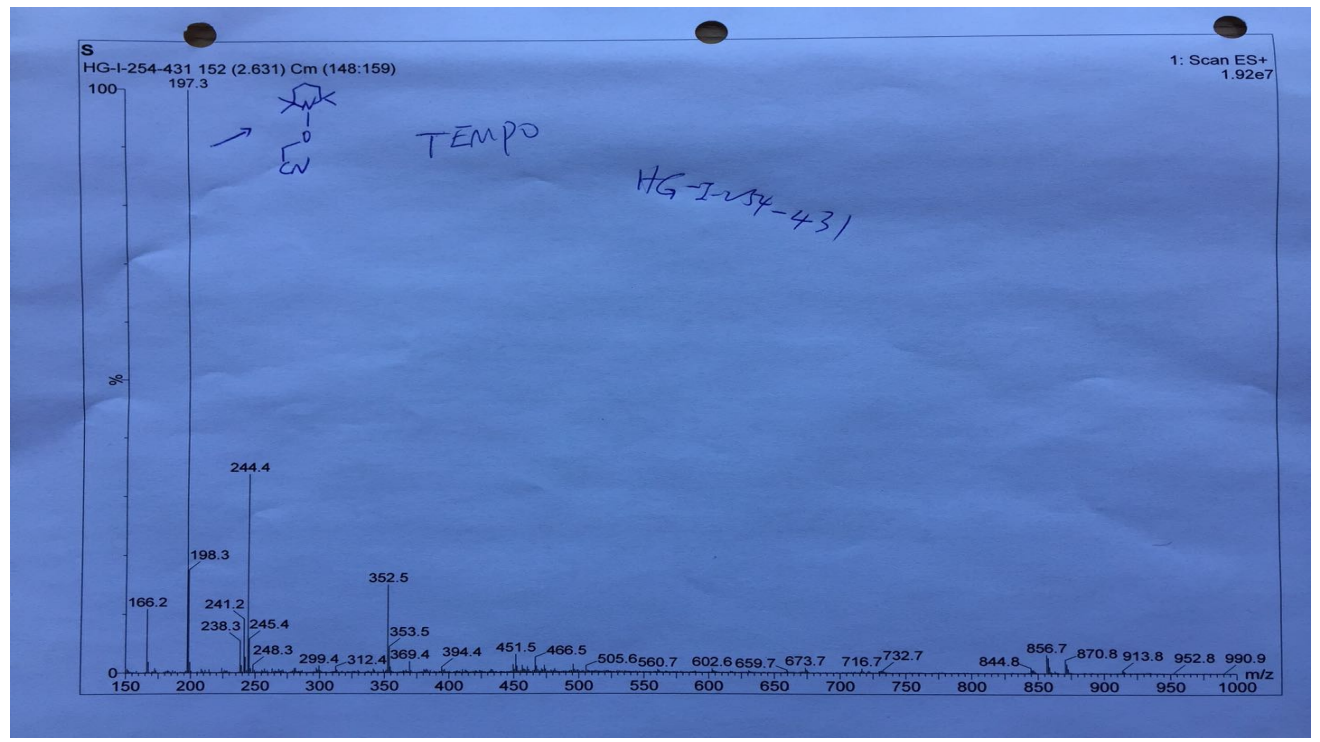

When the BHT (66 mg, 2.0 equiv) was added to the reaction of $5 \mathbf{a}$ with $\mathbf{2 a}$ under the standard conditions, no desired product 6aa was detected, and the BHT-adduct was also observed.

Figure S3.

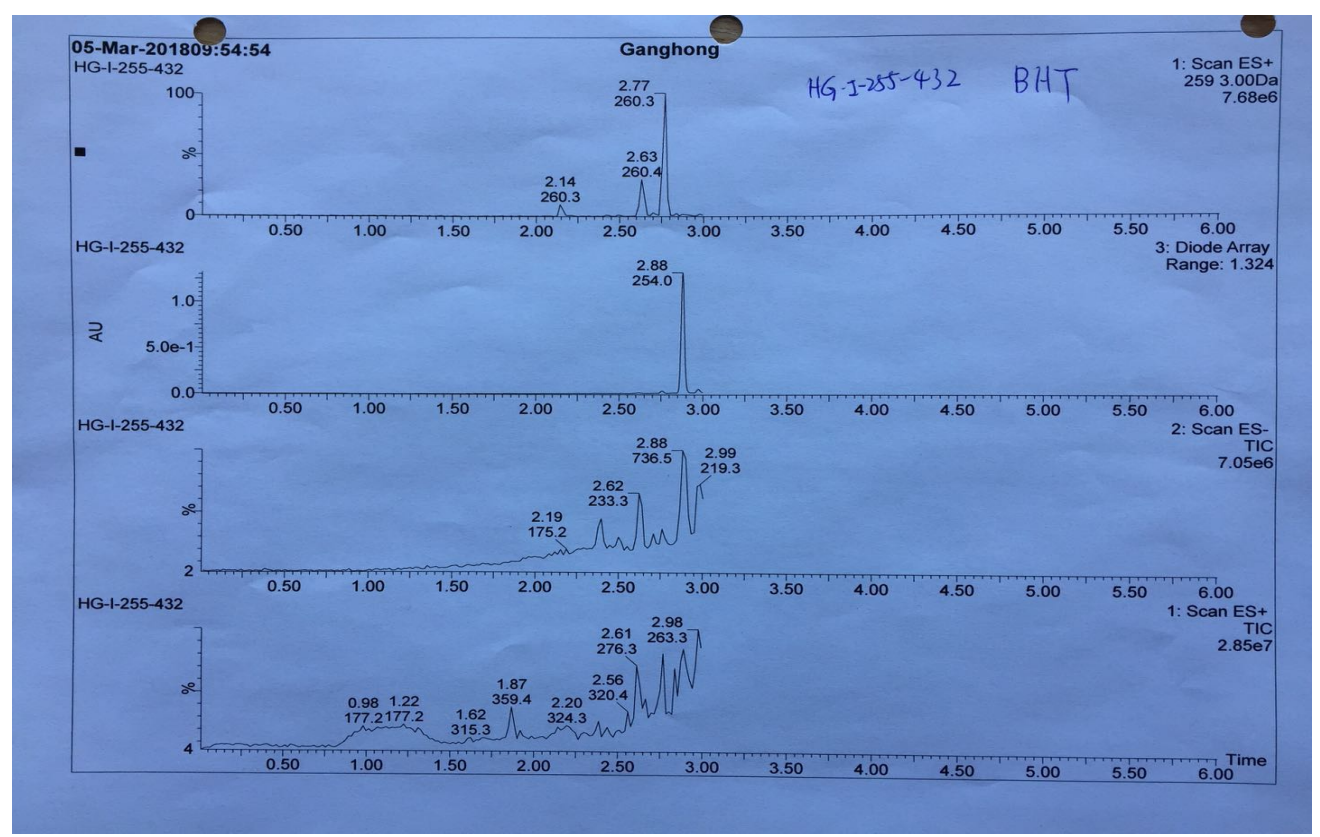


Figure S4.

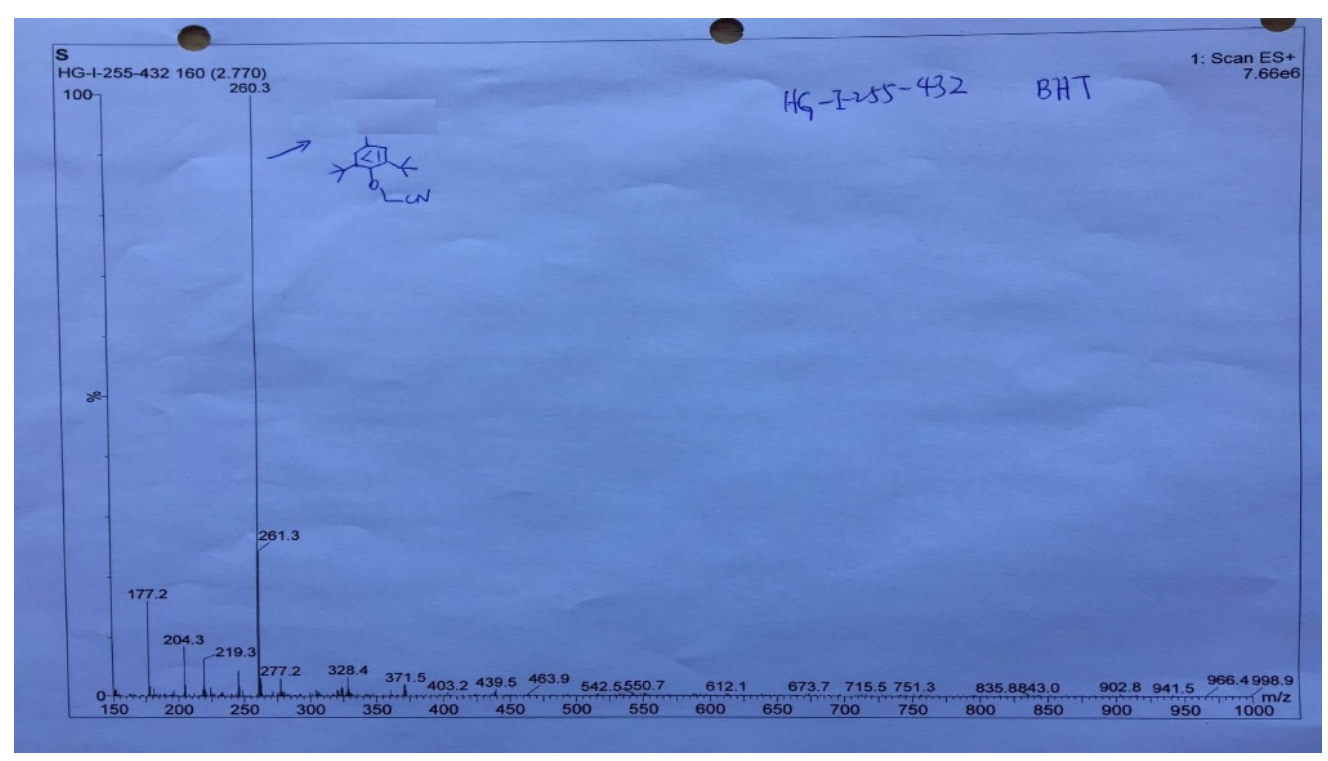

\section{Radical clock experiment.}

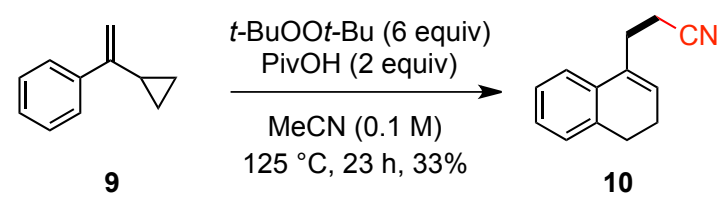

Starting material 9 was synthesized according to literature procedure. ${ }^{4}$ Alkene 9 ( 22 mg, 0.15 mmol) was submitted to the general procedure A. Purification by flash chromatography (100:7 hexane/EtOAc) afforded compound $\mathbf{1 0}$ in $33 \%$ yield $\left(9.1 \mathrm{mg}\right.$ ) as a pale yellow oil. ${ }^{1} \mathrm{H}$ NMR (500 $\left.\mathrm{MHz}, \mathrm{CDCl}_{3}\right) \delta$ 7.24-7.13 (m, 3H), $7.12(\mathrm{~d}, J=7.5 \mathrm{~Hz}, 1 \mathrm{H}), 6.00(\mathrm{t}, J=1.5 \mathrm{~Hz}, 1 \mathrm{H}), 2.84-2.79$ (m, 2H), $2.76(\mathrm{t}, J=8.0 \mathrm{~Hz}, 2 \mathrm{H}), 2.57(\mathrm{t}, J=7.5 \mathrm{~Hz}, 2 \mathrm{H}), 2.32-2.27(\mathrm{~m}, 2 \mathrm{H})$. Spectral data match those previously reported. ${ }^{4}$ 


\section{Competitive KIE experiment.}

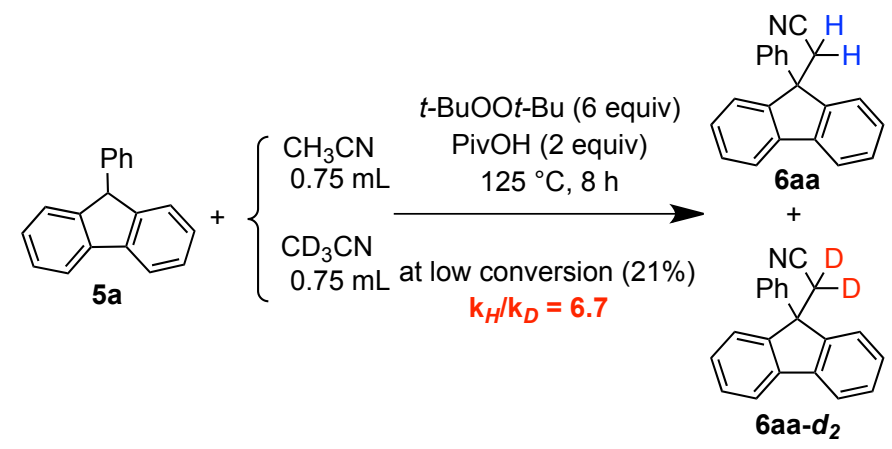

$\mathrm{CD}_{3} \mathrm{CN}(99.9 \%)$ was purchased from Cambridge Isotope Laboratories and was used without further purification. Compound 5a $(36 \mathrm{mg}, 0.15 \mathrm{mmol})$ was added to a flame-dried $8 \mathrm{~mL}$ microwave vial equipped with stir bar and was brought into the glovebox. PivOH (31 mg, 0.30 mmol, 2.0 equiv), acetonitrile/ $\mathrm{D}_{3}$-acetonitrile $(1: 1,1.5 \mathrm{~mL})$, and $t$ - $\mathrm{BuOO} t$ - $\mathrm{Bu}(165 \mu \mathrm{L}, 6.00$ equiv) were added to the mixture sequentially. The microwave vial was sealed with a Teflon cap, removed from the glovebox, and placed in a $125^{\circ} \mathrm{C}$ oil bath. After $8 \mathrm{~h}$, the mixture was allowed to cool to ambient temperature, diluted with $\mathrm{CH}_{2} \mathrm{Cl}_{2}(1 \mathrm{~mL})$, and concentrated. The residue was chromatographed (EtOAc/hexanes) to afford the corresponding product in $21 \%$ yield $(8.8 \mathrm{mg})$. The product was analyzed by ${ }^{1} \mathrm{H}$ NMR (Figure S5):

Figure S5.

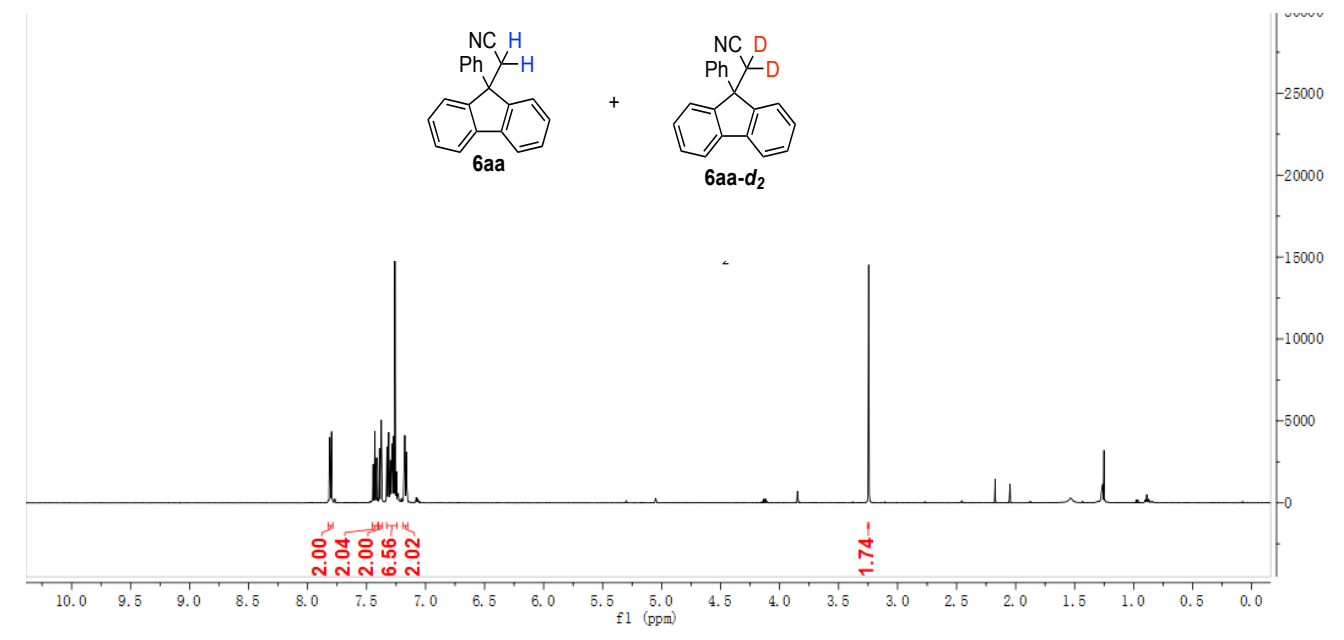




\section{Using dimer intermediates as substrates.}
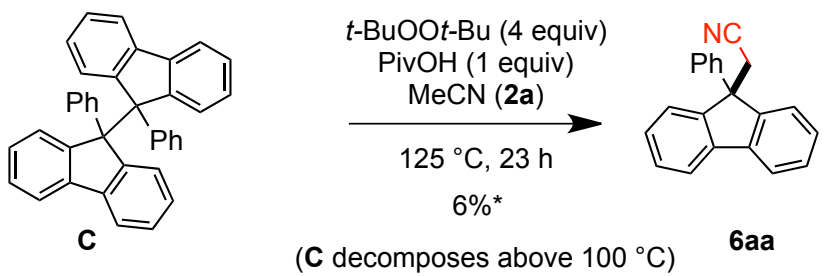

The dimer $(\mathbf{C})$ of fuorene $\mathbf{5 a}(34 \mathrm{mg}, 0.07 \mathrm{mmol})^{5}$ was added to a flame-dried $8 \mathrm{~mL}$ microwave vial equipped with stir bar and was brought into the glovebox. PivOH (7.0 mg, $0.07 \mathrm{mmol}, 1.0$ equiv), acetonitrile $2 \mathrm{a}(0.05 \mathrm{M}, 1.5 \mathrm{~mL})$ and $t$-BuOOt-Bu $(39 \mu \mathrm{L}, 0.28 \mathrm{mmol}, 4.00$ equiv) were added to the mixture sequentially. The microwave vial was sealed with a Teflon cap, removed from the glovebox, and placed in a $125^{\circ} \mathrm{C}$ oil bath. After $23 \mathrm{~h}$, the mixture was allowed to cool to ambient temperature and was concentrated. The mixture was analyzed by ${ }^{1} \mathrm{H}$ NMR spectroscopy use $\mathrm{CH}_{2} \mathrm{Br}_{2}$ as an internal standard: $6 \%$ yield. Dimer $\mathbf{C}$ decomposed above $100{ }^{\circ} \mathrm{C}$ accounting for the low yield.

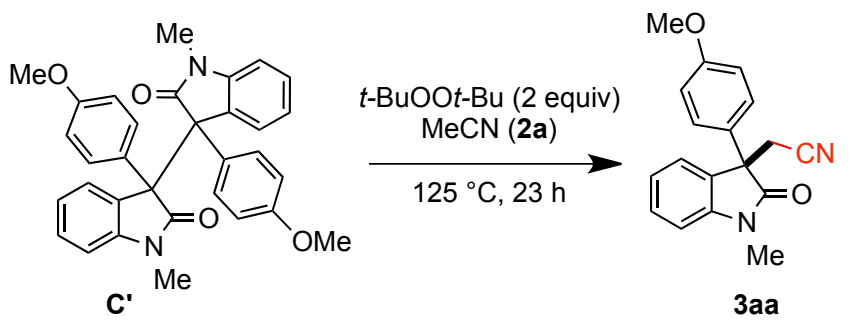

The dimer (C') of oxindole $\mathbf{1 a}(35 \mathrm{mg}, 0.070 \mathrm{mmol})^{6}$ was added to a flame-dried $8 \mathrm{~mL}$ microwave vial equipped with stir bar and was brought into the glovebox. Acetonitrile $2 \mathrm{a}(0.10 \mathrm{M}, 0.7 \mathrm{~mL})$ and $t$-BuOOt-Bu (26 $\mu \mathrm{L}, 0.14 \mathrm{mmol}, 2.00$ equiv) were added to the mixture sequentially. The microwave vial was sealed with a Teflon cap, removed from the glovebox, and placed in a $130{ }^{\circ} \mathrm{C}$ oil bath. After $24 \mathrm{~h}$, the mixture was allowed to cool to ambient temperature and was concentrated. The mixture was analyzed by ${ }^{1} \mathrm{H}$ NMR spectroscopy use $\mathrm{CH}_{2} \mathrm{Br}_{2}$ as an internal standard: $20 \%$ yield. 


\subsection{Characterization Data of Products}

\section{2-(1-Methyl-2-oxo-3-phenylindolin-3-yl)acetonitrile (3aa)}<smiles>CN1C(=O)C(CC#N)(c2ccccc2)c2ccccc21</smiles>

General procedure A was followed using $1 \mathrm{a}(34 \mathrm{mg}, 0.15 \mathrm{mmol})$, and $t$-BuOOt -Bu $(110 \mu \mathrm{L}, 4.00$ equiv) in acetonitrile 2a $(1.5 \mathrm{~mL}, 0.10 \mathrm{M})$ at $130{ }^{\circ} \mathrm{C}$ for $24 \mathrm{~h}$. Chromatography (12\% EtOAc/hexanes) afforded 3aa in 48\% yield (19 mg) as an amorphous brown solid: ${ }^{1} \mathrm{H}$ NMR (500 $\left.\mathrm{MHz}, \mathrm{CDCl}_{3}\right) \delta 7.53(\mathrm{~d}, J=7.5 \mathrm{~Hz}, 1 \mathrm{H}), 7.44(\mathrm{td}, J=8.0,1.5 \mathrm{~Hz}, 1 \mathrm{H}), 7.36-7.30(\mathrm{~m}, 5 \mathrm{H}), 7.22(\mathrm{t}$, $J=7.5 \mathrm{~Hz}, 1 \mathrm{H}), 6.98(\mathrm{~d}, J=8.0 \mathrm{~Hz}, 1 \mathrm{H}), 3.38(\mathrm{~d}, J=17.0 \mathrm{~Hz}, 1 \mathrm{H}), 3.25(\mathrm{~s}, 3 \mathrm{H}), 3.05(\mathrm{~d}, J=17.0$ $\mathrm{Hz}, 1 \mathrm{H}) ;{ }^{13} \mathrm{C} \mathrm{NMR}\left(125 \mathrm{MHz}, \mathrm{CDCl}_{3}\right) \delta 175.6,143.5,136.4,129.6,129.3,128.9,128.3,126.7$, 125.2, 123.3, 116.3, 108.9, 52.6, 26.7, 26.3; IR (film) 2925, 2250, 1714, 1611, 1471, 1371, 755, $696 \mathrm{~cm}^{-1}$; HRMS (EI-TOF) calcd for $\mathrm{C}_{17} \mathrm{H}_{14} \mathrm{~N}_{2} \mathrm{O}[\mathrm{M}]^{+} \mathrm{m} / \mathrm{z}=262.1106$; found 262.1098 .

\section{2-(1-Methyl-2-oxo-3-(p-tolyl)indolin-3-yl)acetonitrile (3ba)}

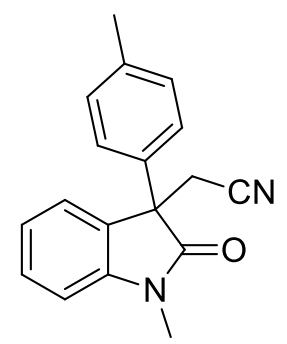

General procedure A was followed using $1 \mathbf{b}(36 \mathrm{mg}, 0.15 \mathrm{mmol})$, and $t$-BuOOt-Bu $(110 \mu \mathrm{L}, 4.00$ equiv) in acetonitrile $2 \mathrm{a}(1.5 \mathrm{~mL}, 0.10 \mathrm{M})$ at $130{ }^{\circ} \mathrm{C}$ for $24 \mathrm{~h}$. Chromatography (12\% EtOAc/hexanes) afforded 3ba in $38 \%$ yield $(16 \mathrm{mg})$ as a colorless oil: ${ }^{1} \mathrm{H} \mathrm{NMR}\left(500 \mathrm{MHz}, \mathrm{CDCl}_{3}\right)$ $\delta 7.52(\mathrm{~d}, J=7.5 \mathrm{~Hz}, 1 \mathrm{H}), 7.44(\mathrm{td}, J=7.5,1.0 \mathrm{~Hz}, 1 \mathrm{H}), 7.23-7.19(\mathrm{~m}, 3 \mathrm{H}), 7.14(\mathrm{~d}, J=8.0 \mathrm{~Hz}$, 2H), $6.97(\mathrm{~d}, J=8.0 \mathrm{~Hz}, 1 \mathrm{H}), 3.37(\mathrm{~d}, J=16.5 \mathrm{~Hz}, 1 \mathrm{H}), 3.24(\mathrm{~s}, 3 \mathrm{H}), 3.02(\mathrm{~d}, J=16.5 \mathrm{~Hz}, 1 \mathrm{H})$, 
$2.32(\mathrm{~s}, 3 \mathrm{H}) ;{ }^{13} \mathrm{C} \mathrm{NMR}\left(125 \mathrm{MHz}, \mathrm{CDCl}_{3}\right) \delta$ 176.0, 143.7, 138.4, 133.6, 129.9, 129.8, 129.7, 126.8, 125.3, 123.5, 116.7, 109.1, 52.5, 26.9, 26.5, 21.1; IR (film) 2924, 2249, 1713, 1611, 1470, 1371, 754, $690 \mathrm{~cm}^{-1}$; HRMS (EI-TOF) calcd for $\mathrm{C}_{18} \mathrm{H}_{16} \mathrm{~N}_{2} \mathrm{O}[\mathrm{M}]^{+} \mathrm{m} / \mathrm{z}=276.1263$; found 276.1261 .

\section{2-(3-(4-Methoxyphenyl)-1-methyl-2-oxoindolin-3-yl)acetonitrile (3ca)}

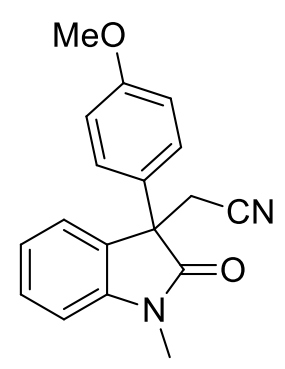

General procedure A was followed using $1 \mathbf{c}(38 \mathrm{mg}, 0.15 \mathrm{mmol})$, and $t$-BuOOt-Bu $(110 \mu \mathrm{L}, 4.00$ equiv) in acetonitrile 2a $(1.5 \mathrm{~mL}, 0.10 \mathrm{M})$ at $130{ }^{\circ} \mathrm{C}$ for $24 \mathrm{~h}$. Chromatography $(16 \%$ EtOAc/hexanes) afforded $3 \mathbf{c a}$ in $46 \%$ yield $\left(20 \mathrm{mg}\right.$ ) as a colorless oil: ${ }^{1} \mathrm{H}$ NMR ( $\left.500 \mathrm{MHz}, \mathrm{CDCl}_{3}\right)$ $\delta 7.54(\mathrm{~d}, J=7.5 \mathrm{~Hz}, 1 \mathrm{H}), 7.43(\mathrm{td}, J=7.5,1.0 \mathrm{~Hz}, 1 \mathrm{H}), 7.29-7.26(\mathrm{~m}, 2 \mathrm{H}), 7.22(\mathrm{t}, J=7.0 \mathrm{~Hz}$, 1H), $6.97(\mathrm{~d}, J=8.0 \mathrm{~Hz}, 1 \mathrm{H}), 6.86(\mathrm{~d}, J=9.0 \mathrm{~Hz}, 2 \mathrm{H}), 3.78(\mathrm{~s}, 3 \mathrm{H}), 3.34(\mathrm{~d}, J=16.5 \mathrm{~Hz}, 1 \mathrm{H})$, $3.23(\mathrm{~s}, 3 \mathrm{H}), 2.98(\mathrm{~d}, J=16.5 \mathrm{~Hz}, 1 \mathrm{H}) ;{ }^{13} \mathrm{C} \mathrm{NMR}\left(125 \mathrm{MHz}, \mathrm{CDCl}_{3}\right) \delta 176.1,159.7,143.7,129.8$, 129.7, 128.5, 128.2, 125.4, 123.5, 116.6, 114.5, 109.1, 55.4, 52.2, 26.8, 26.7; IR (film) 2958, 2249, 1713, 1610, 1470, 1371, 1254, $754 \mathrm{~cm}^{-1}$; HRMS (EI-TOF) calcd for $\mathrm{C}_{18} \mathrm{H}_{16} \mathrm{~N}_{2} \mathrm{O}_{2}[\mathrm{M}]^{+} \mathrm{m} / \mathrm{z}=$ 292.1212; found 292.1209.

2-(3-(4-Fluorophenyl)-1-methyl-2-oxoindolin-3-yl)acetonitrile (3da)<smiles>CN1C(=O)C(CC#N)(c2ccc(F)cc2)c2ccccc21</smiles> 
General procedure A was followed using $1 \mathbf{d}(36 \mathrm{mg}, 0.15 \mathrm{mmol})$, and $t$-BuOOt-Bu $(110 \mu \mathrm{L}, 4.00$ equiv) in acetonitrile 2a $(1.5 \mathrm{~mL}, 0.10 \mathrm{M})$ at $130{ }^{\circ} \mathrm{C}$ for $24 \mathrm{~h}$. Chromatography $(12 \%$ EtOAc/hexanes) afforded 3da in 40\% yield (17 mg) as a colorless oil: ${ }^{1} \mathrm{H}$ NMR $\left(500 \mathrm{MHz}, \mathrm{CDCl}_{3}\right)$ $\delta 7.55(\mathrm{~d}, J=7.5 \mathrm{~Hz}, 1 \mathrm{H}), 7.45(\mathrm{td}, J=8.0,1.0 \mathrm{~Hz}, 1 \mathrm{H}), 7.37-7.34(\mathrm{~m}, 2 \mathrm{H}), 7.24$ (t, $J=7.5 \mathrm{~Hz}$, 1H), $7.03(\mathrm{t}, J=8.5 \mathrm{~Hz}, 2 \mathrm{H}), 6.98(\mathrm{~d}, J=7.5 \mathrm{~Hz}, 1 \mathrm{H}), 3.35(\mathrm{~d}, J=16.5 \mathrm{~Hz}, 1 \mathrm{H}), 3.24(\mathrm{~s}, 3 \mathrm{H}), 2.98$ $(\mathrm{d}, J=16.5 \mathrm{~Hz}, 1 \mathrm{H}) ;{ }^{13} \mathrm{C} \mathrm{NMR}\left(125 \mathrm{MHz}, \mathrm{CDCl}_{3}\right) \delta 175.9,163.1(\mathrm{~d}, J=247 \mathrm{~Hz}), 143.9,132.5$ (d, $J=3.1 \mathrm{~Hz}), 130.3,129.5,129.1(\mathrm{~d}, J=8.3 \mathrm{~Hz}), 125.6,123.9,116.6,116.3(\mathrm{~d}, J=21.6 \mathrm{~Hz})$, 109.5, 52.4, 27.2, 27.1; ${ }^{19} \mathrm{~F}$ NMR (376 MHz, $\mathrm{CDCl}_{3}$ ) $\delta$-113.5; IR (film) 2925, 2250, 1713, 1612, 1471, 1371, 1235, $754 \mathrm{~cm}^{-1}$; HRMS (EI-TOF) calcd for $\mathrm{C}_{17} \mathrm{H}_{13} \mathrm{FN}_{2} \mathrm{O}[\mathrm{M}]^{+} \mathrm{m} / \mathrm{z}=280.1012$; found 280.1021.

\section{2-(1-Methyl-2-oxo-3-(m-tolyl)indolin-3-yl)acetonitrile (3ea)}

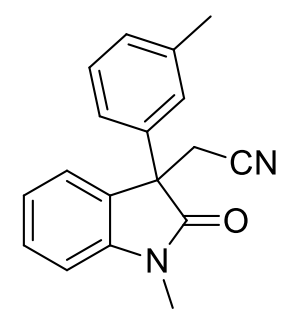

General procedure A was followed using $1 \mathrm{e}(36 \mathrm{mg}, 0.15 \mathrm{mmol})$, and $t$-BuOOt-Bu $(110 \mu \mathrm{L}, 4.00$ equiv) in acetonitrile 2a $(1.5 \mathrm{~mL}, 0.10 \mathrm{M})$ at $130{ }^{\circ} \mathrm{C}$ for $24 \mathrm{~h}$. Chromatography $(12 \%$ EtOAc/hexanes) afforded 3ea in $47 \%$ yield $\left(20 \mathrm{mg}\right.$ ) as an amorphous white solid: ${ }^{1} \mathrm{H}$ NMR (500 $\left.\mathrm{MHz}, \mathrm{CDCl}_{3}\right) \delta 7.51(\mathrm{~d}, J=7.5 \mathrm{~Hz}, 1 \mathrm{H}), 7.44(\mathrm{td}, J=7.5,1.0 \mathrm{~Hz}, 1 \mathrm{H}), 7.24-7.19(\mathrm{~m}, 2 \mathrm{H}), 7.15-$ $7.09(\mathrm{~m}, 3 \mathrm{H}), 6.97(\mathrm{~d}, J=8.0 \mathrm{~Hz}, 1 \mathrm{H}), 3.36(\mathrm{~d}, J=17.0 \mathrm{~Hz}, 1 \mathrm{H}), 3.25(\mathrm{~s}, 3 \mathrm{H}), 3.04(\mathrm{~d}, J=17.0$ $\mathrm{Hz}, 1 \mathrm{H}), 2.32(\mathrm{~s}, 3 \mathrm{H}) ;{ }^{13} \mathrm{C} \mathrm{NMR}\left(125 \mathrm{MHz}, \mathrm{CDCl}_{3}\right) \delta 176.0,143.7,138.9,136.6,129.7,129.3$, 128.9, 127.4, 125.3, 123.9, 123.5, 118. 0, 116.6, 109.1, 52.8, 26.9, 26.5, 21.7; IR (film) 2932, 2250, 
1713, 1611, 1470, 1370, 754, $732 \mathrm{~cm}^{-1}$; HRMS (EI-TOF) calcd for $\mathrm{C}_{18} \mathrm{H}_{16} \mathrm{~N}_{2} \mathrm{O}[\mathrm{M}]^{+} \mathrm{m} / \mathrm{z}=$ 276.1263; found 276.1279 .

2-(1,5-Dimethyl-2-oxo-3-phenylindolin-3-yl)acetonitrile (3fa)

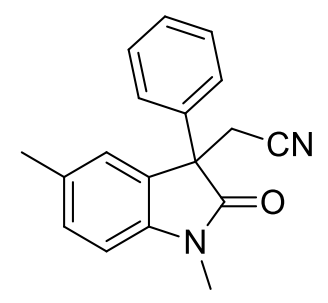

General procedure A was followed using $\mathbf{1 f}(36 \mathrm{mg}, 0.15 \mathrm{mmol})$, and $t$-BuOOt-Bu $(110 \mu \mathrm{L}, 4.00$ equiv) in acetonitrile 2a $(1.5 \mathrm{~mL}, 0.10 \mathrm{M})$ at $130{ }^{\circ} \mathrm{C}$ for $24 \mathrm{~h}$. Chromatography $(12 \%$ EtOAc/hexanes) afforded $3 \mathbf{f a}$ in $40 \%$ yield $\left(17 \mathrm{mg}\right.$ ) as a colorless oil: ${ }^{1} \mathrm{H} \mathrm{NMR}\left(500 \mathrm{MHz}, \mathrm{CDCl}_{3}\right)$ $\delta 7.35-7.30(\mathrm{~m}, 6 \mathrm{H}), 7.23(\mathrm{~d}, J=8.0 \mathrm{~Hz}, 1 \mathrm{H}), 6.86(\mathrm{~d}, J=8.0 \mathrm{~Hz}, 1 \mathrm{H}), 3.36(\mathrm{~d}, J=16.5 \mathrm{~Hz}, 1 \mathrm{H})$, $3.23(\mathrm{~s}, 3 \mathrm{H}), 3.03(\mathrm{~d}, J=16.5 \mathrm{~Hz}, 1 \mathrm{H}), 2.41(\mathrm{~s}, 3 \mathrm{H}) ;{ }^{13} \mathrm{C} \mathrm{NMR}\left(125 \mathrm{MHz}, \mathrm{CDCl}_{3}\right) \delta 175.8,141.4$, $136.8,133.2,130.1,129.7,129.1,128.4,126.9,126.0,116.6,108.8,52.9,26.9,26.5,21.4$; IR (film) 2923, 2250, 1712, 1619, 1499, 1355, 734, $697 \mathrm{~cm}^{-1}$; HRMS (EI-TOF) calcd for $\mathrm{C}_{18} \mathrm{H}_{16} \mathrm{~N}_{2} \mathrm{O}$ $[\mathrm{M}]^{+} \mathrm{m} / \mathrm{z}=276.1263$; found 276.1265 .

\section{2-(6-Chloro-1-methyl-2-oxo-3-phenylindolin-3-yl)acetonitrile (3ga)}<smiles>CN1C(=O)C(CC#N)(c2ccccc2)c2ccc(Cl)cc21</smiles>

General procedure A was followed using $1 \mathrm{~g}$ (39 $\mathrm{mg}, 0.15 \mathrm{mmol})$, and $t$-BuOOt-Bu $(110 \mu \mathrm{L}, 4.00$ equiv) in acetonitrile $2 \mathrm{a}(1.5 \mathrm{~mL}, 0.10 \mathrm{M})$ at $130{ }^{\circ} \mathrm{C}$ for $24 \mathrm{~h}$. Chromatography $(11 \%$ EtOAc/hexanes) afforded 3ga in $63 \%$ yield $\left(28 \mathrm{mg}\right.$ ) as an amorphous white solid: ${ }^{1} \mathrm{H}$ NMR (500 $\left.\mathrm{MHz}, \mathrm{CDCl}_{3}\right) \delta 7.44(\mathrm{~d}, J=8.0 \mathrm{~Hz}, 1 \mathrm{H}), 7.37-7.30(\mathrm{~m}, 5 \mathrm{H}), 7.20(\mathrm{dd}, J=8.0,2.0 \mathrm{~Hz}, 1 \mathrm{H}), 6.98$ 
$(\mathrm{d}, J=1.5 \mathrm{~Hz}, 1 \mathrm{H}), 3.36(\mathrm{~d}, J=17.0 \mathrm{~Hz}, 1 \mathrm{H}), 3.23(\mathrm{~s}, 3 \mathrm{H}), 3.05(\mathrm{~d}, J=17.0 \mathrm{~Hz}, 1 \mathrm{H}) ;{ }^{13} \mathrm{C}$ NMR $\left(125 \mathrm{MHz}, \mathrm{CDCl}_{3}\right) \delta 175.8,144.9,136.1,135.9,129.2,128.8,127.8,126.7,126.3,123.4,116.3$, 109.9, 52.6, 27.0, 26.5; IR (film) 2948, 2250, 1719, 1608, 1494, 1367, 729, $695 \mathrm{~cm}^{-1}$; HRMS (EITOF) calcd for $\mathrm{C}_{17} \mathrm{H}_{13} \mathrm{ClN}_{2} \mathrm{O}[\mathrm{M}]^{+} \mathrm{m} / \mathrm{z}=296.0716$; found 296.0719 .

\section{2-(1-Benzyl-2-oxo-3-phenylindolin-3-yl)acetonitrile (3ha)}<smiles>N#CC1(c2ccccc2)C(=O)N(Cc2ccccc2)c2ccccc21</smiles>

General procedure A was followed using $1 \mathbf{h}(45 \mathrm{mg}, 0.15 \mathrm{mmol})$, and $t$-BuOOt-Bu (110 $\mu \mathrm{L}, 4.00$ equiv) in acetonitrile $2 \mathrm{a}(1.5 \mathrm{~mL}, 0.10 \mathrm{M})$ at $130{ }^{\circ} \mathrm{C}$ for $24 \mathrm{~h}$. Chromatography $(13 \%$ EtOAc/hexanes) afforded 3 ha in $52 \%$ yield $\left(26 \mathrm{mg}\right.$ ) as a colorless oil: ${ }^{1} \mathrm{H} \mathrm{NMR}\left(500 \mathrm{MHz}, \mathrm{CDCl}_{3}\right)$ $\delta 7.50(\mathrm{~d}, J=7.5 \mathrm{~Hz}, 1 \mathrm{H}), 7.38-7.24(\mathrm{~m}, 11 \mathrm{H}), 7.17(\mathrm{td}, J=7.5,1.0 \mathrm{~Hz}, 1 \mathrm{H}), 6.85(\mathrm{~d}, J=8.0 \mathrm{~Hz}$ 1H), $4.96(\mathrm{~d}, J=16.0 \mathrm{~Hz}, 1 \mathrm{H}), 4.92(\mathrm{~d}, J=16.0 \mathrm{~Hz}, 1 \mathrm{H}), 3.44(\mathrm{~d}, J=16.5 \mathrm{~Hz}, 1 \mathrm{H}), 3.15(\mathrm{~d}, J=$ $16.5 \mathrm{~Hz}, 1 \mathrm{H}) ;{ }^{13} \mathrm{C} \mathrm{NMR}\left(125 \mathrm{MHz}, \mathrm{CDCl}_{3}\right) \delta$ 176.1, 142.9, 136.8, 135.3, 129.7 (2C), 129.2, 129.0, 128.6, 127.9, 127.3, 126.8, 125.3, 123.6, 116.5, 110.2, 52.9, 44.4, 26.4; IR (film) 3032, 2925, 2250, 1713, 1612, 1487, 1362, 732, $696 \mathrm{~cm}^{-1}$; HRMS (EI-TOF) calcd for $\mathrm{C}_{23} \mathrm{H}_{18} \mathrm{~N}_{2} \mathrm{O}[\mathrm{M}]^{+} \mathrm{m} / \mathrm{z}=$ 338.1419 ; found 338.1433 .

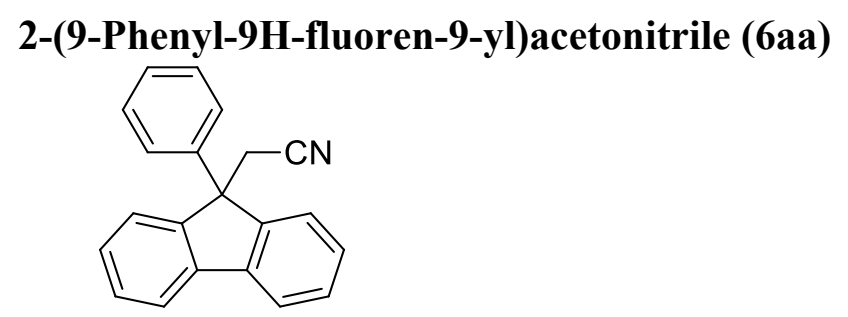

General procedure B was followed using 5a (36 mg, $0.15 \mathrm{mmol})$, PivOH (31 mg, $0.30 \mathrm{mmol}, 2.0$ equiv), and $t$-BuOOt-Bu (165 $\mu \mathrm{L}, 6.00$ equiv) in acetonitrile $(1.5 \mathrm{~mL}, 0.10 \mathrm{M})$ at $125^{\circ} \mathrm{C}$ for $23 \mathrm{~h}$. 
Chromatography (4\% EtOAc/hexanes) afforded 6aa $(27 \mathrm{mg})$ in $65 \%$ yield as a colorless oil: ${ }^{1} \mathrm{H}$ $\operatorname{NMR}\left(500 \mathrm{MHz}, \mathrm{CDCl}_{3}\right) \delta 7.80(\mathrm{~d}, J=7.5 \mathrm{~Hz}, 2 \mathrm{H}), 7.43(\mathrm{td}, J=7.5,1.0 \mathrm{~Hz}, 2 \mathrm{H}), 7.39(\mathrm{~d}, J=7.5$ $\mathrm{Hz}, 2 \mathrm{H}), 7.34-7.23(\mathrm{~m}, 5 \mathrm{H}), 7.17(\mathrm{~d}, J=7.0 \mathrm{~Hz}, 2 \mathrm{H}), 3.25(\mathrm{~s}, 2 \mathrm{H}) ;{ }^{13} \mathrm{C} \mathrm{NMR}\left(125 \mathrm{MHz}, \mathrm{CDCl}_{3}\right)$ $\delta 149.4,140.9,140.1,129.0,128.7,128.3,127.6,126.4,124.6,120.7,117.7,55.5,27.7$; IR (film) 3061, 2926, 2250, 1698, 1449, 754, $733 \mathrm{~cm}^{-1}$; HRMS (EI-TOF) calcd for $\mathrm{C}_{21} \mathrm{H}_{15} \mathrm{~N}[\mathrm{M}]^{+} \mathrm{m} / \mathrm{z}=$ 281.1204; found 281.1195.

\section{2-(9-(p-Tolyl)-9H-fluoren-9-yl)acetonitrile (6ba)}

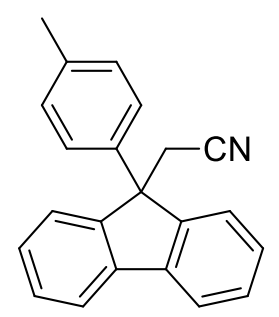

General procedure B was followed using $\mathbf{5 b}(38.5 \mathrm{mg}, 0.15 \mathrm{mmol})$, PivOH (31 mg, $0.30 \mathrm{mmol}$, 2.0 equiv), and $t$ - $\mathrm{BuOO} t$ - $\mathrm{Bu}\left(165 \mu \mathrm{L}, 6.00\right.$ equiv) in acetonitrile $(1.5 \mathrm{~mL}, 0.10 \mathrm{M})$ at $125^{\circ} \mathrm{C}$ for $23 \mathrm{~h}$. Chromatography (4\% EtOAc/hexanes) afforded $\mathbf{6 b a}$ in $61 \%$ yield $(27.0 \mathrm{mg})$ as a colorless oil: ${ }^{1} \mathrm{H}$ NMR (500 MHz, $\left.\mathrm{CDCl}_{3}\right) \delta 7.80(\mathrm{~d}, J=7.5 \mathrm{~Hz}, 2 \mathrm{H}), 7.42(\mathrm{td}, J=7.5,1.0 \mathrm{~Hz}, 2 \mathrm{H}), 7.38(\mathrm{~d}$, $J=7.5 \mathrm{~Hz}, 2 \mathrm{H}), 7.31(\mathrm{td}, J=7.5,1.0 \mathrm{~Hz}, 2 \mathrm{H}), 7.08(\mathrm{~d}, J=8.0 \mathrm{~Hz}, 2 \mathrm{H}), 7.05(\mathrm{~d}, J=8.5 \mathrm{~Hz}, 2 \mathrm{H})$, $3.22(\mathrm{~s}, 2 \mathrm{H}), 2.30(\mathrm{~s}, 3 \mathrm{H}) ;{ }^{13} \mathrm{C} \mathrm{NMR}\left(125 \mathrm{MHz}, \mathrm{CDCl}_{3}\right) \delta$ 149.5, 140.1, 137.9, 137.3, 129.7, 128.6, 128.3, 126.3, 124.5, 120.6, 117.7, 55.3, 27.8, 21.1; IR (film) 3024, 2922, 2248, 1512, 1448, 748 , $734 \mathrm{~cm}^{-1}$; HRMS (EI-TOF) calcd for $\mathrm{C}_{22} \mathrm{H}_{17} \mathrm{~N}[\mathrm{M}]^{+} \mathrm{m} / \mathrm{z}=295.1361$; found 295.1364 .

\section{2-(9-(4-Methoxyphenyl)-9H-fluoren-9-yl)acetonitrile (6ca)}

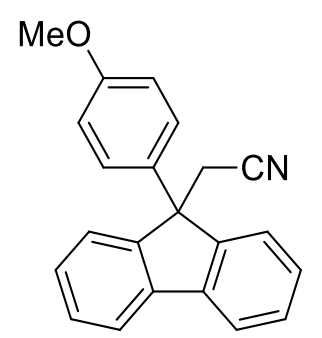


General procedure B was followed using 5c (41 mg, $0.15 \mathrm{mmol})$, PivOH (31 mg, $0.30 \mathrm{mmol}, 2.0$ equiv), and $t$-BuOOt-Bu (165 $\mu \mathrm{L}, 6.00$ equiv) in acetonitrile $(1.5 \mathrm{~mL}, 0.10 \mathrm{M})$ at $125^{\circ} \mathrm{C}$ for $23 \mathrm{~h}$. Chromatography (7\% EtOAc/hexanes) afforded $6 \mathbf{c a}$ in $55 \%$ yield $(26 \mathrm{mg})$ as a colorless oil: ${ }^{1} \mathrm{H}$ $\operatorname{NMR}\left(500 \mathrm{MHz}, \mathrm{CDCl}_{3}\right) \delta 7.79(\mathrm{~d}, J=7.5 \mathrm{~Hz}, 2 \mathrm{H}), 7.44-7.37(\mathrm{~m}, 4 \mathrm{H}), 7.31(\mathrm{td}, J=7.5,1.0 \mathrm{~Hz}$, 2H), $7.09(\mathrm{~d}, J=9.0 \mathrm{~Hz}, 2 \mathrm{H}), 6.81(\mathrm{~d}, J=9.0 \mathrm{~Hz}, 2 \mathrm{H}), 3.77(\mathrm{~s}, 3 \mathrm{H}), 3.21(\mathrm{~s}, 2 \mathrm{H}) ;{ }^{13} \mathrm{C}$ NMR $(125$ $\left.\mathrm{MHz}, \mathrm{CDCl}_{3}\right) \delta 158.9,149.6,140.0,132.8,128.6,128.3,127.5,124.5,120.6,117.7,114.3,55.4$, 54.9, 27.9; IR (film) 3031, 2932, 2249, 1580, 1448, 1185, 749, $735 \mathrm{~cm}^{-1}$; HRMS (EI-TOF) calcd for $\mathrm{C}_{22} \mathrm{H}_{17} \mathrm{NO}[\mathrm{M}]^{+} \mathrm{m} / \mathrm{z}=311.1310$; found 311.1310 .

\section{2-(9-(4-(tert-Butyl)phenyl)-9H-fluoren-9-yl)acetonitrile (6da)}

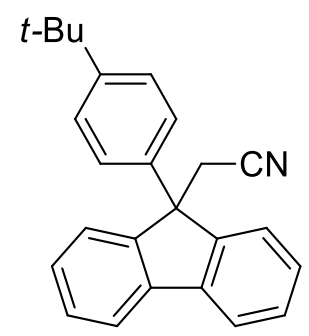

General procedure B was followed using 5d (45 mg, $0.15 \mathrm{mmol})$, PivOH (31 mg, $0.30 \mathrm{mmol}, 2.0$ equiv), and $t$-BuOOt-Bu $\left(165 \mu \mathrm{L}, 6.00\right.$ equiv) in acetonitrile $(1.5 \mathrm{~mL}, 0.10 \mathrm{M})$ at $125^{\circ} \mathrm{C}$ for $23 \mathrm{~h}$. Chromatography (6\% EtOAc/hexanes) afforded $\mathbf{6 d a}$ in $62 \%$ yield $(31 \mathrm{mg})$ as a colorless oil: ${ }^{1} \mathrm{H}$ NMR (500 MHz, $\left.\mathrm{CDCl}_{3}\right) \delta 7.80(\mathrm{~d}, J=7.5 \mathrm{~Hz}, 2 \mathrm{H}), 7.44-7.39(\mathrm{~m}, 4 \mathrm{H}), 7.33-7.26(\mathrm{~m}, 4 \mathrm{H}), 7.09$ (d, $J=8.5 \mathrm{~Hz}, 2 \mathrm{H}), 3.24$ (s, 2H), 1.27 (s, 9H); ${ }^{13} \mathrm{C} \mathrm{NMR}\left(125 \mathrm{MHz}, \mathrm{CDCl}_{3}\right) \delta$ 150.4, 149.4, 140.1, 137.7, 128.6, 128.2, 126.0, 125.9, 124.6, 120.6, 117.7, 55.2, 34.5, 31.4, 27.8; IR (film) 3025, 2962, 2248, 1448, 758, $734 \mathrm{~cm}^{-1}$; HRMS (EI-TOF) calcd for $\mathrm{C}_{25} \mathrm{H}_{23} \mathrm{~N}[\mathrm{M}]^{+} \mathrm{m} / \mathrm{z}=337.1830$; found 337.1830.

\section{2-(9-(4-Chlorophenyl)-9H-fluoren-9-yl)acetonitrile (6ea)}




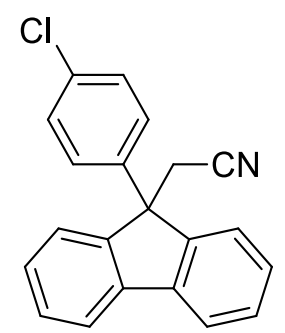

General procedure B was followed using 5e (42 mg, $0.15 \mathrm{mmol})$, PivOH (31 mg, $0.30 \mathrm{mmol}, 2.0$ equiv), and $t$-BuOOt-Bu (165 $\mu \mathrm{L}, 6.00$ equiv) in acetonitrile $(1.5 \mathrm{~mL}, 0.10 \mathrm{M})$ at $125^{\circ} \mathrm{C}$ for $23 \mathrm{~h}$. Chromatography (5\% EtOAc/hexanes) afforded 6ea in 55\% yield $(26 \mathrm{mg})$ as a colorless oil: ${ }^{1} \mathrm{H}$ $\operatorname{NMR}\left(500 \mathrm{MHz}, \mathrm{CDCl}_{3}\right) \delta 7.80(\mathrm{~d}, J=7.5 \mathrm{~Hz}, 2 \mathrm{H}), 7.44(\mathrm{td}, J=7.5,1.0 \mathrm{~Hz}, 2 \mathrm{H}), 7.37-7.30(\mathrm{~m}$, 4H), $7.25(\mathrm{~d}, J=7.5 \mathrm{~Hz}, 2 \mathrm{H}), 7.10(\mathrm{~d}, J=8.5 \mathrm{~Hz}, 2 \mathrm{H}), 3.20(\mathrm{~s}, 2 \mathrm{H}) ;{ }^{13} \mathrm{C} \mathrm{NMR}\left(125 \mathrm{MHz}, \mathrm{CDCl}_{3}\right)$ $\delta 148.9,140.0,139.5,133.6,129.2,128.9,128.5,127.9,124.4,120.8,117.4,55.1,27.7$; IR (film) $3065,2925,2250,1492,1448,1094,758,735 \mathrm{~cm}^{-1}$; HRMS (EI-TOF) calcd for $\mathrm{C}_{21} \mathrm{H}_{14} \mathrm{ClN}[\mathrm{M}]^{+}$ $\mathrm{m} / \mathrm{z}=315.0815$; found 315.0796 .

\section{2-(9-(4-Fluorophenyl)-9H-fluoren-9-yl)acetonitrile (6fa)}

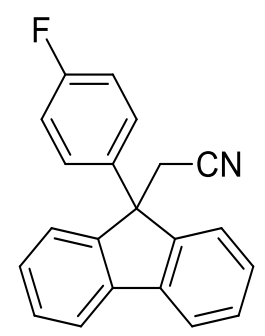

General procedure B was followed using $\mathbf{5 f}$ (39 mg, $0.15 \mathrm{mmol}$ ), PivOH (31 mg, $0.30 \mathrm{mmol}, 2.0$ equiv), and $t$-BuOOt-Bu (165 $\mu \mathrm{L}, 6.00$ equiv) in acetonitrile $(1.5 \mathrm{~mL}, 0.10 \mathrm{M})$ at $125^{\circ} \mathrm{C}$ for $23 \mathrm{~h}$. Chromatography (4\% EtOAc/hexanes) afforded $6 \mathbf{f a}$ in $45 \%$ yield $(20 \mathrm{mg})$ as a colorless oil: ${ }^{1} \mathrm{H}$ $\operatorname{NMR}\left(500 \mathrm{MHz}, \mathrm{CDCl}_{3}\right) \delta 7.79(\mathrm{~d}, J=7.5 \mathrm{~Hz}, 2 \mathrm{H}), 7.42(\mathrm{td}, J=7.5,1.0 \mathrm{~Hz}, 2 \mathrm{H}), 7.37-7.34(\mathrm{~m}$, $2 \mathrm{H}), 7.30(\mathrm{td}, J=7.5,1.0 \mathrm{~Hz}, 2 \mathrm{H}), 7.15-7.11(\mathrm{~m}, 2 \mathrm{H}), 6.95(\mathrm{t}, J=8.5 \mathrm{~Hz}, 2 \mathrm{H}), 3.19(\mathrm{~s}, 2 \mathrm{H}) ;{ }^{13} \mathrm{C}$ $\operatorname{NMR}\left(125 \mathrm{MHz}, \mathrm{CDCl}_{3}\right) \delta 161.9(\mathrm{~d}, J=246 \mathrm{~Hz}), 148.9,139.8,136.5(\mathrm{~d}, J=3.5 \mathrm{~Hz}), 128.6,128.2$, $127.9(\mathrm{~d}, J=8.3 \mathrm{~Hz}), 124.2,120.5,117.2,115.6(\mathrm{~d}, J=21.4 \mathrm{~Hz}), 54.8,27.7 ;{ }^{19} \mathrm{~F}$ NMR $(376 \mathrm{MHz}$, 
$\mathrm{CDCl}_{3}$ ) $\delta$-114.9; IR (film) 3065, 2923, 2249, 1508, 1449, 750, $734 \mathrm{~cm}^{-1}$; HRMS (EI-TOF) calcd for $\mathrm{C}_{21} \mathrm{H}_{14} \mathrm{FN}[\mathrm{M}]^{+} \mathrm{m} / \mathrm{z}=299.1110$; found 299.1115 .

2-(9-(4-(Trifluoromethyl)phenyl)-9H-fluoren-9-yl)acetonitrile (6ga)

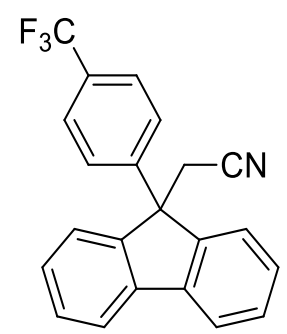

General procedure B was followed using $\mathbf{5 g}(46 \mathrm{mg}, 0.15 \mathrm{mmol})$, PivOH (31 mg, $0.30 \mathrm{mmol}, 2.0$ equiv), and $t$-BuOOt-Bu (165 $\mu \mathrm{L}, 6.00$ equiv) in acetonitrile $(1.5 \mathrm{~mL}, 0.10 \mathrm{M})$ at $125^{\circ} \mathrm{C}$ for $23 \mathrm{~h}$. Chromatography (4\% EtOAc/hexanes) afforded 6ga in 46\% yield (24 mg) as a white solid: ${ }^{1} \mathrm{H}$ $\operatorname{NMR}\left(500 \mathrm{MHz}, \mathrm{CDCl}_{3}\right) \delta 7.83(\mathrm{~d}, J=7.5 \mathrm{~Hz}, 2 \mathrm{H}), 7.54(\mathrm{~d}, J=8.5 \mathrm{~Hz}, 2 \mathrm{H}), 7.46(\operatorname{td}, J=7.0,1.5$ $\mathrm{Hz}, 2 \mathrm{H}), 7.38-7.31(\mathrm{~m}, 4 \mathrm{H}), 7.29(\mathrm{~d}, J=8.5 \mathrm{~Hz}, 2 \mathrm{H}), 3.25(\mathrm{~s}, 2 \mathrm{H}) ;{ }^{13} \mathrm{C} \mathrm{NMR}\left(125 \mathrm{MHz}, \mathrm{CDCl}_{3}\right)$ $\delta 148.5,145.1,139.9,129.9$ (q, $J=32.4 \mathrm{~Hz}), 128.9,128.4,126.8,125.8(\mathrm{q}, J=3.6 \mathrm{~Hz}), 124.9$ (q, $J=271 \mathrm{~Hz}), 124.3,120.7,117.2,55.3,27.4 ;{ }^{19} \mathrm{~F} \mathrm{NMR}\left(376 \mathrm{MHz}, \mathrm{CDCl}_{3}\right) \delta$-62.7; IR (film) 3066, 2926, 2249, 1618, 1328, 1119, 750, $733 \mathrm{~cm}^{-1}$; HRMS (EI-TOF) calcd for $\mathrm{C}_{22} \mathrm{H}_{14} \mathrm{~F}_{3} \mathrm{~N}[\mathrm{M}]^{+} \mathrm{m} / \mathrm{z}=$ 349.1078; found 349.1093 .

\section{2-(9-([1,1'-Biphenyl]-4-yl)-9H-fluoren-9-yl)acetonitrile (6ha)}

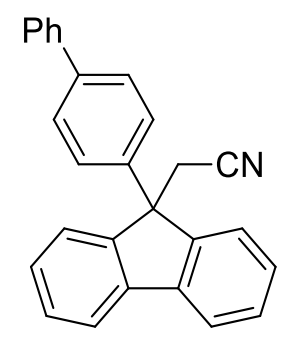

General procedure B was followed using $\mathbf{5 h}(48 \mathrm{mg}, 0.15 \mathrm{mmol})$, PivOH (31 mg, $0.30 \mathrm{mmol}, 2.0$ equiv), and $t$-BuOOt-Bu (165 $\mu \mathrm{L}, 6.00$ equiv) in acetonitrile $(1.5 \mathrm{~mL}, 0.10 \mathrm{M})$ at $125^{\circ} \mathrm{C}$ for $23 \mathrm{~h}$. Chromatography (5\% EtOAc/hexanes) afforded 6 ha in $47 \%$ yield $(25 \mathrm{mg})$ as a white solid: ${ }^{1} \mathrm{H}$ 
$\operatorname{NMR}\left(500 \mathrm{MHz}, \mathrm{CDCl}_{3}\right) \delta 7.80(\mathrm{~d}, J=7.5 \mathrm{~Hz}, 2 \mathrm{H}), 7.53-7.47(\mathrm{~m}, 4 \mathrm{H}), 7.45-7.38(\mathrm{~m}, 6 \mathrm{H}), 7.34-$ $7.29(\mathrm{~m}, 3 \mathrm{H}), 7.22(\mathrm{~d}, J=8.5 \mathrm{~Hz}, 2 \mathrm{H}), 3.26(\mathrm{~s}, 2 \mathrm{H}) ;{ }^{13} \mathrm{C} \mathrm{NMR}\left(125 \mathrm{MHz}, \mathrm{CDCl}_{3}\right) \delta$ 149.1, 140.3, $140.2,139.9,137.9,128.7,128.5,128.2,127.5,127.3,126.9,126.6,124.4,120.5,117.4,55.1$, 27.6; IR (film) 3030, 2924, 2248, 1486, 1448, 762, $735 \mathrm{~cm}^{-1}$; HRMS (EI-TOF) calcd for $\mathrm{C}_{27} \mathrm{H}_{19} \mathrm{~N}$ $[\mathrm{M}]^{+} \mathrm{m} / \mathrm{z}=357.1517$; found 357.1502 .

\section{2-(9-(3-methoxyphenyl)-9H-fluoren-9-yl)acetonitrile (6ia)}

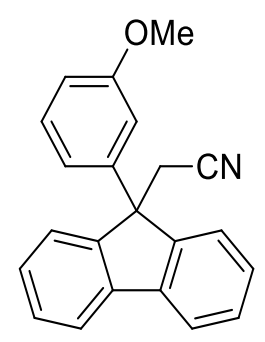

General procedure B was followed using $\mathbf{5 i}$ (41 mg, $0.15 \mathrm{mmol}$ ), PivOH ( $31 \mathrm{mg}, 0.30 \mathrm{mmol}, 2.0$ equiv), and $t$-BuOOt-Bu (165 $\mu \mathrm{L}, 6.00$ equiv) in acetonitrile $(1.5 \mathrm{~mL}, 0.10 \mathrm{M})$ at $125^{\circ} \mathrm{C}$ for $23 \mathrm{~h}$. Chromatography (7\% EtOAc/hexanes) afforded 6ia in 51\% yield (24 mg) as a colorless oil: ${ }^{1} \mathrm{H}$ $\operatorname{NMR}\left(500 \mathrm{MHz}, \mathrm{CDCl}_{3}\right) \delta 7.79(\mathrm{~d}, J=7.5 \mathrm{~Hz}, 2 \mathrm{H}), 7.44-7.39(\mathrm{~m}, 4 \mathrm{H}), 7.31(\mathrm{td}, J=7.5,1.0 \mathrm{~Hz}$, 2H), 7.22 (t, $J=8.0 \mathrm{~Hz}, 1 \mathrm{H}), 6.79$ (td, $J=7.0,1.5 \mathrm{~Hz}, 2 \mathrm{H}), 6.67$ (t, $J=2.5 \mathrm{~Hz}, 1 \mathrm{H}), 3.71(\mathrm{~s}, 3 \mathrm{H})$, $3.22(\mathrm{~s}, 2 \mathrm{H}) ;{ }^{13} \mathrm{C} \mathrm{NMR}\left(125 \mathrm{MHz}, \mathrm{CDCl}_{3}\right) \delta 159.9,149.1,142.6,140.1,129.9,128.7,128.3,124.5$, 120.6, 118.7, 117.6, 113.3, 112.0, 55.5, 55.3, 27.8; IR (film) 3067, 2948, 2247, 1490, 1449, 766, $734 \mathrm{~cm}^{-1}$; HRMS (EI-TOF) calcd for $\mathrm{C}_{22} \mathrm{H}_{17} \mathrm{NO}[\mathrm{M}]^{+} \mathrm{m} / \mathrm{z}=311.1310$; found 311.1298 .

\section{2-(9-(3-Fluorophenyl)-9H-fluoren-9-yl)acetonitrile (5ja)}

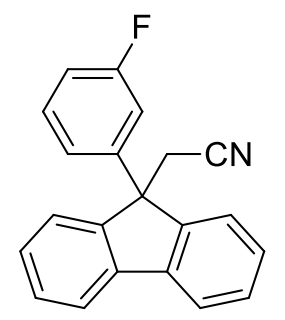


General procedure B was followed using $5 \mathbf{j}$ (39 mg, $0.15 \mathrm{mmol}$ ), PivOH (31 mg, $0.30 \mathrm{mmol}, 2.0$ equiv), and $t$-BuOOt-Bu (165 $\mu \mathrm{L}, 6.00$ equiv) in acetonitrile $(1.5 \mathrm{~mL}, 0.10 \mathrm{M})$ at $125^{\circ} \mathrm{C}$ for $23 \mathrm{~h}$. Chromatography (3\% EtOAc/hexanes) afforded $5 \mathbf{j a}$ in $61 \%$ yield $(27 \mathrm{mg})$ as a colorless oil: ${ }^{1} \mathrm{H}$ $\operatorname{NMR}\left(500 \mathrm{MHz}, \mathrm{CDCl}_{3}\right) \delta 7.81(\mathrm{~d}, J=7.5 \mathrm{~Hz}, 2 \mathrm{H}), 7.45(\mathrm{td}, J=7.5,1.0 \mathrm{~Hz}, 2 \mathrm{H}), 7.39(\mathrm{~d}, J=7.5$ $\mathrm{Hz}, 2 \mathrm{H}), 7.35-7.27(\mathrm{~m}, 3 \mathrm{H}), 7.05-7.02(\mathrm{~m}, 1 \mathrm{H}), 6.98-6.93(\mathrm{~m}, 1 \mathrm{H}), 6.80(\mathrm{dt}, J=10.0,2.5 \mathrm{~Hz}, 1 \mathrm{H})$, $3.22(\mathrm{~s}, 2 \mathrm{H}) ;{ }^{13} \mathrm{C} \mathrm{NMR}\left(125 \mathrm{MHz}, \mathrm{CDCl}_{3}\right) \delta 163.1(\mathrm{~d}, J=245 \mathrm{~Hz}), 148.7,143.6(\mathrm{~d}, J=6.9 \mathrm{~Hz})$, $140.1,130.5$ (d, $J=8.4 \mathrm{~Hz}), 128.9,128.5,124.5,121.9$ (d, $J=2.8 \mathrm{~Hz}), 120.8,117.3,114.6(\mathrm{~d}, J$ $=21.0 \mathrm{~Hz}), 113.8(\mathrm{~d}, J=22.8 \mathrm{~Hz}), 55.3(\mathrm{~d}, J=1.8 \mathrm{~Hz}), 28.8(\mathrm{~d}, J=269 \mathrm{~Hz}) ;{ }^{19} \mathrm{~F}$ NMR $(376 \mathrm{MHz}$, $\mathrm{CDCl}_{3}$ ) $\delta$-111.7; IR (film) 2923, 2852, 2249, 1588, 1449, 764, $748 \mathrm{~cm}^{-1}$; HRMS (EI-TOF) calcd for $\mathrm{C}_{21} \mathrm{H}_{14} \mathrm{FN}[\mathrm{M}]^{+} \mathrm{m} / \mathrm{z}=299.1110$; found 299.1118 .

\section{2-(9-(Benzo[d][1,3]dioxol-5-yl)-9H-fluoren-9-yl)acetonitrile (6ka)}

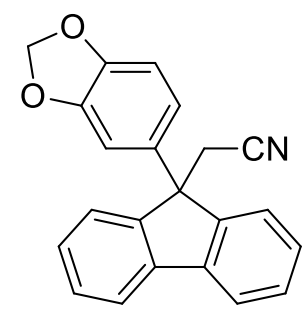

General procedure B was followed using 5k (43 mg, $0.15 \mathrm{mmol}$ ), PivOH ( $31 \mathrm{mg}, 0.30 \mathrm{mmol}, 2.0$ equiv), and $t$-BuOOt-Bu (165 $\mu \mathrm{L}, 6.00$ equiv) in acetonitrile $(1.5 \mathrm{~mL}, 0.10 \mathrm{M})$ at $125^{\circ} \mathrm{C}$ for $23 \mathrm{~h}$. Chromatography (7\% EtOAc/hexanes) afforded $\mathbf{6 k a}$ in $34 \%$ yield $(17 \mathrm{mg})$ as a colorless oil: ${ }^{1} \mathrm{H}$ NMR $\left(500 \mathrm{MHz}, \mathrm{CDCl}_{3}\right) \delta 7.78(\mathrm{~d}, J=8.0 \mathrm{~Hz}, 2 \mathrm{H}), 7.44-7.38(\mathrm{~m}, 4 \mathrm{H}), 7.31(\mathrm{td}, J=8.5,1.0 \mathrm{~Hz}$, 2H), $6.82(\mathrm{dd}, J=8.5,2.0 \mathrm{~Hz}, 1 \mathrm{H}), 6.75(\mathrm{~d}, J=8.5 \mathrm{~Hz}, 1 \mathrm{H}), 6.43(\mathrm{~d}, J=2.0 \mathrm{~Hz}, 1 \mathrm{H}), 5.89(\mathrm{~s}, 2 \mathrm{H})$, $3.17(\mathrm{~s}, 2 \mathrm{H}) ;{ }^{13} \mathrm{C} \mathrm{NMR}\left(125 \mathrm{MHz}, \mathrm{CDCl}_{3}\right) \delta$ 149.3, 148.3, 147.1, 139.9, 134.7, 128.7, 128.3, 124.4, 120.7, 119.3, 117.6, 108.4, 107.4, 101.3, 55.3, 27.9; IR (film) 3062, 2919, 2249, 1488, 1448, 1240 , 1040, 752, $735 \mathrm{~cm}^{-1}$; HRMS (EI-TOF) calcd for $\mathrm{C}_{22} \mathrm{H}_{15} \mathrm{NO}_{2}[\mathrm{M}]^{+} \mathrm{m} / \mathrm{z}=325.1103$; found 325.1122 . 2-(9-(Naphthalen-1-yl)-9H-fluoren-9-yl)acetonitrile (6la) 


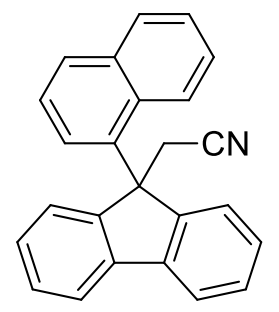

General procedure B was followed using $5 \mathbf{l}$ (44 mg, $0.15 \mathrm{mmol}$ ), PivOH (31 mg, $0.30 \mathrm{mmol}, 2.0$ equiv), and $t$-BuOOt-Bu (165 $\mu \mathrm{L}, 6.00$ equiv) in acetonitrile $(1.5 \mathrm{~mL}, 0.10 \mathrm{M})$ at $125^{\circ} \mathrm{C}$ for $23 \mathrm{~h}$. Chromatography ( $5 \%$ EtOAc/hexanes) afforded 6la in 36\% yield $(18 \mathrm{mg})$ as an amorphous white solid: ${ }^{1} \mathrm{H}$ NMR (500 MHz, $\left.\mathrm{CDCl}_{3}\right) \delta 7.99(\mathrm{~d}, J=7.5 \mathrm{~Hz}, 1 \mathrm{H}), 7.95(\mathrm{~d}, J=7.5 \mathrm{~Hz}, 2 \mathrm{H}), 7.86(\mathrm{~d}, J$ $=8.5 \mathrm{~Hz}, 1 \mathrm{H}), 7.75(\mathrm{~d}, J=8.0 \mathrm{~Hz}, 1 \mathrm{H}), 7.63(\mathrm{t}, J=7.5 \mathrm{~Hz}, 1 \mathrm{H}), 7.46(\mathrm{t}, J=7.5 \mathrm{~Hz}, 2 \mathrm{H}), 7.22(\mathrm{t}$, $J=7.5 \mathrm{~Hz}, 3 \mathrm{H}), 7.14(\mathrm{~d}, J=7.5 \mathrm{~Hz}, 2 \mathrm{H}), 6.84(\mathrm{t}, J=8.5 \mathrm{~Hz}, 1 \mathrm{H}), 6.57(\mathrm{~d}, J=9.0 \mathrm{~Hz}, 1 \mathrm{H}), 3.14$ (s, 2H); ${ }^{13} \mathrm{C}$ NMR $\left(125 \mathrm{MHz}, \mathrm{CDCl}_{3}\right) \delta 150.5,139.5,134.8,130.8,129.7,128.9,128.7,128.5$, 127.6, 127.5, 125.9, 125.8, 125.5, 125.3, 124.9, 124.1, 121.3, 56.3, 31.9; IR (film) 3051, 2923, 2249, 1448, 775, $736 \mathrm{~cm}^{-1}$; HRMS (EI-TOF) calcd for $\mathrm{C}_{25} \mathrm{H}_{17} \mathrm{~N}[\mathrm{M}]^{+} \mathrm{m} / \mathrm{z}=331.1361$; found 331.1367.

\section{2-(9-(4-(9H-Carbazol-9-yl)phenyl)-9H-fluoren-9-yl)acetonitrile (5ma)}

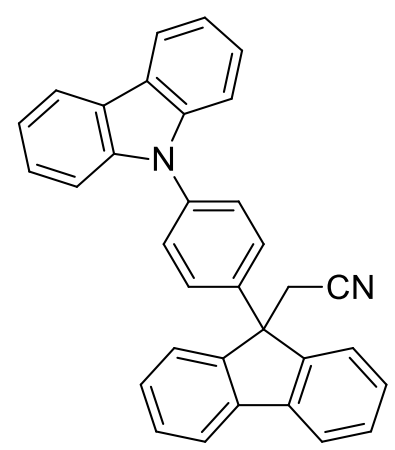

General procedure B was followed using $5 \mathbf{m}(61 \mathrm{mg}, 0.15 \mathrm{mmol})$, PivOH (31 mg, $0.30 \mathrm{mmol}, 2.0$ equiv), and $t$-BuOOt-Bu (165 $\mu \mathrm{L}, 6.00$ equiv) in acetonitrile $(1.5 \mathrm{~mL}, 0.10 \mathrm{M})$ at $125^{\circ} \mathrm{C}$ for $23 \mathrm{~h}$. Chromatography (6\% EtOAc/hexanes) afforded $5 \mathrm{ma}$ in $49 \%$ yield (33 $\mathrm{mg}$ ) an amorphous white 
solid: ${ }^{1} \mathrm{H}$ NMR $\left(500 \mathrm{MHz}, \mathrm{CDCl}_{3}\right) \delta 8.12(\mathrm{~d}, J=8.0 \mathrm{~Hz}, 2 \mathrm{H}), 7.85(\mathrm{~d}, J=7.5 \mathrm{~Hz}, 2 \mathrm{H}), 7.53-7.47$ (m, 6H), 7.43-7.36 (m, 8H), 7.30-7.26 (m, 2H), $3.32(\mathrm{~s}, 2 \mathrm{H}) ;{ }^{13} \mathrm{C} \mathrm{NMR}\left(125 \mathrm{MHz}, \mathrm{CDCl}_{3}\right) \delta$ 149.1, $140.8,140.2,140.1,137.2,128.9,128.5,127.9,127.4,126.1,124.6,123.6,120.8,120.4,120.2$, 117.5, 109.9, 55.4, 27.9; IR (film) 3061, 2925, 2249, 1514, 1451, 1229, 750, $735 \mathrm{~cm}^{-1}$; HRMS (EITOF) calcd for $\mathrm{C}_{33} \mathrm{H}_{22} \mathrm{~N}_{2}[\mathrm{M}]^{+} \mathrm{m} / \mathrm{z}=446.1783$; found 446.1793 .

\section{2-(9-Butyl-9H-fluoren-9-yl)acetonitrile (6na)}

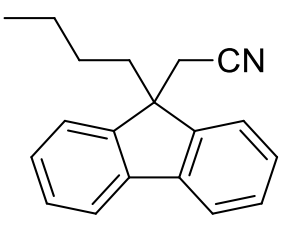

General procedure B was followed using $5 \mathbf{n}(33 \mathrm{mg}, 0.15 \mathrm{mmol})$, PivOH ( $31 \mathrm{mg}, 0.30 \mathrm{mmol}, 2.0$ equiv), and $t$-BuOOt-Bu (165 $\mu \mathrm{L}, 6.00$ equiv) in acetonitrile $(1.5 \mathrm{~mL}, 0.10 \mathrm{M})$ at $125^{\circ} \mathrm{C}$ for $23 \mathrm{~h}$. Chromatography (4\% EtOAc/hexanes) afforded 6 na in $51 \%$ yield $(20 \mathrm{mg})$ as a colorless oil: ${ }^{1} \mathrm{H}$ $\operatorname{NMR}\left(500 \mathrm{MHz}, \mathrm{CDCl}_{3}\right) \delta 7.74(\mathrm{~d}, J=7.5 \mathrm{~Hz}, 2 \mathrm{H}), 7.54(\mathrm{~d}, J=7.5 \mathrm{~Hz}, 2 \mathrm{H}), 7.41(\mathrm{td}, J=7.5,1.0$ $\mathrm{Hz}, 2 \mathrm{H}), 7.35$ (td, $J=7.5,1.5 \mathrm{~Hz}, 2 \mathrm{H}), 2.72(\mathrm{~s}, 2 \mathrm{H}), 2.23-2.19$ (m, 2H), 1.14 (q, $J=7.5 \mathrm{~Hz}, 2 \mathrm{H})$, $0.70(\mathrm{t}, J=7.5 \mathrm{~Hz}, 3 \mathrm{H}), 0.67-0.60(\mathrm{~m}, 2 \mathrm{H}) ;{ }^{13} \mathrm{C} \mathrm{NMR}\left(125 \mathrm{MHz}, \mathrm{CDCl}_{3}\right) \delta 147.5,140.5,128.4$, 127.9, 123.1, 120.4, 117.9, 51.0, 37.2, 29.3, 26.0, 22.8, 13.8; IR (film) 2956, 2930, 2860, 2247, 1449, 761, $736 \mathrm{~cm}^{-1}$; HRMS (EI-TOF) calcd for $\mathrm{C}_{19} \mathrm{H}_{19} \mathrm{~N}[\mathrm{M}]^{+} \mathrm{m} / \mathrm{z}=261.1517$; found 261.1542 . 2-(2-Fluoro-9-phenyl-9H-fluoren-9-yl)acetonitrile (6oa)

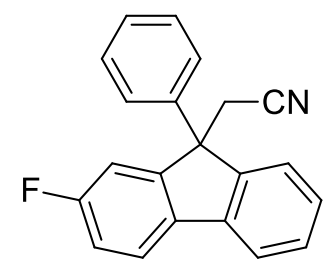

General procedure B was followed using 50 (39 mg, $0.15 \mathrm{mmol}$ ), PivOH (31 mg, $0.30 \mathrm{mmol}, 2.0$ equiv), and $t$-BuOOt-Bu $\left(165 \mu \mathrm{L}, 6.00\right.$ equiv) in acetonitrile $(1.5 \mathrm{~mL}, 0.10 \mathrm{M})$ at $125^{\circ} \mathrm{C}$ for $23 \mathrm{~h}$. 
Chromatography (4\% EtOAc/hexanes) afforded 6oa in 34\% yield (15 mg) as a colorless oil: ${ }^{1} \mathrm{H}$ NMR $\left(500 \mathrm{MHz}, \mathrm{CDCl}_{3}\right) \delta$ 7.77-7.73 (m, 2H), $7.43(\mathrm{t}, J=7.5 \mathrm{~Hz}, 1 \mathrm{H}), 7.36(\mathrm{~d}, J=7.5 \mathrm{~Hz}, 1 \mathrm{H})$, 7.32-7.26 (m, 4H), 7.17-7.11 (m, 3H), 7.07 (dd, $J=8.5,2.5 \mathrm{~Hz}, 1 \mathrm{H}), 3.25(\mathrm{~s}, 2 \mathrm{H}) ;{ }^{13} \mathrm{C}$ NMR $(125$ $\left.\mathrm{MHz}, \mathrm{CDCl}_{3}\right) \delta 163.0(\mathrm{~d}, J=246 \mathrm{~Hz}), 151.5(\mathrm{~d}, J=7.6 \mathrm{~Hz}), 149.3,140.3,139.3,136.2,129.1$, 128.9, 128.0, 127.8, 126.3, 124.5, $121.8(\mathrm{~d}, J=8.8 \mathrm{~Hz}), 120.4,117.2,116.0(\mathrm{~d}, J=23.0 \mathrm{~Hz}), 112.1$ (d, $J=23.3 \mathrm{~Hz}), 55.6,27.6 ;{ }^{19} \mathrm{~F}$ NMR $\left(376 \mathrm{MHz}, \mathrm{CDCl}_{3}\right) \delta-112.4$; IR (film) 3052, 2248, 1592, 1454, 1265, 757, $732 \mathrm{~cm}^{-1}$; HRMS (EI-TOF) calcd for $\mathrm{C}_{21} \mathrm{H}_{14} \mathrm{FN}[\mathrm{M}]^{+} \mathrm{m} / \mathrm{z}=299.1110$; found 299.1120.

\section{2-(9-(4-Methoxyphenyl)-2-nitro-9H-fluoren-9-yl)acetonitrile (6pa)}

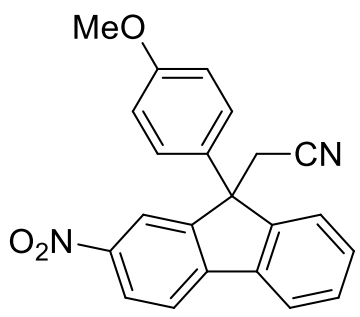

General procedure B was followed using 5p (48 mg, $0.15 \mathrm{mmol}$ ), PivOH ( $31 \mathrm{mg}, 0.30 \mathrm{mmol}, 2.0$ equiv), and $t$-BuOOt-Bu $\left(165 \mu \mathrm{L}, 6.00\right.$ equiv) in acetonitrile $(1.5 \mathrm{~mL}, 0.10 \mathrm{M})$ at $125^{\circ} \mathrm{C}$ for $23 \mathrm{~h}$. Chromatography (12\% EtOAc/hexanes) afforded 6pa in 55\% yield (29 $\mathrm{mg})$ an amorphous white solid: ${ }^{1} \mathrm{H}$ NMR (500 MHz, $\left.\mathrm{CDCl}_{3}\right) \delta 8.33(\mathrm{dd}, J=8.0,2.0 \mathrm{~Hz}, 1 \mathrm{H}), 8.16(\mathrm{~d}, J=1.0 \mathrm{~Hz}, 1 \mathrm{H}), 7.92-$ $7.88(\mathrm{~m}, 2 \mathrm{H}), 7.54-7.45(\mathrm{~m}, 3 \mathrm{H}), 7.06(\mathrm{~d}, J=9.0 \mathrm{~Hz}, 2 \mathrm{H}), 6.83(\mathrm{~d}, J=9.0 \mathrm{~Hz}, 2 \mathrm{H}), 3.78(\mathrm{~s}, 3 \mathrm{H})$, $3.42(\mathrm{~d}, J=16.5 \mathrm{~Hz}, 1 \mathrm{H}), 3.25(\mathrm{~d}, J=16.5 \mathrm{~Hz}, 1 \mathrm{H}) ;{ }^{13} \mathrm{C} \mathrm{NMR}\left(125 \mathrm{MHz}, \mathrm{CDCl}_{3}\right) \delta 159.5,150.8$, $150.7,147.8,146.4,137.9,131.1,130.5,129.3,127.4,124.9,124.8,122.1,120.9,119.9,116.7$, 114.8, 55.4, 55.4, 27.7; IR (film) 3062, 2250, 1592, 1454, 1265, 757, $732 \mathrm{~cm}^{-1}$; HRMS (EI-TOF) calcd for $\mathrm{C}_{22} \mathrm{H}_{16} \mathrm{~N}_{2} \mathrm{O}_{3}[\mathrm{M}]^{+} \mathrm{m} / \mathrm{z}=356.1161$; found 356.1176 .

\section{2-(2,7-Dibromo-9-(4-methoxyphenyl)-9H-fluoren-9-yl)acetonitrile (6qa)}




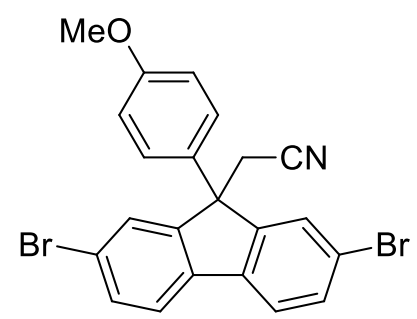

General procedure B was followed using $\mathbf{5 q}(64 \mathrm{mg}, 0.15 \mathrm{mmol})$, PivOH (31 mg, $0.30 \mathrm{mmol}, 2.0$ equiv), and $t$-BuOOt-Bu (165 $\mu \mathrm{L}, 6.00$ equiv) in acetonitrile $(1.5 \mathrm{~mL}, 0.10 \mathrm{M})$ at $125^{\circ} \mathrm{C}$ for $23 \mathrm{~h}$. Chromatography (5\% EtOAc/hexanes) afforded 6qa in 64\% yield (45 mg) as an amorphous white solid: ${ }^{1} \mathrm{H}$ NMR (500 MHz, $\left.\mathrm{CDCl}_{3}\right) \delta 7.62(\mathrm{~d}, J=8.0 \mathrm{~Hz}, 2 \mathrm{H}), 7.55(\mathrm{dd}, J=8.0,1.5 \mathrm{~Hz}, 2 \mathrm{H}), 7.46$ (d, $J=1.5 \mathrm{~Hz}, 2 \mathrm{H}), 7.02(\mathrm{~d}, J=9.0 \mathrm{~Hz}, 2 \mathrm{H}), 6.84(\mathrm{~d}, J=9.0 \mathrm{~Hz}, 2 \mathrm{H}), 3.79(\mathrm{~s}, 3 \mathrm{H}), 3.23(\mathrm{~s}, 2 \mathrm{H})$; ${ }^{13} \mathrm{C}$ NMR $\left(125 \mathrm{MHz}, \mathrm{CDCl}_{3}\right) \delta 159.4,151.2,138.1,132.2,131.2,127.8,127.4,122.5,122.1$, 116.8, 114.7, 55.4, 55.2, 27.7; IR (film) 2931, 2836, 2249, 1512, 1253, $735 \mathrm{~cm}^{-1}$; HRMS (EI-TOF) calcd for $\mathrm{C}_{22} \mathrm{H}_{15} \mathrm{Br}_{2} \mathrm{NO}[\mathrm{M}]^{+} \mathrm{m} / \mathrm{z}=466.9520$; found 466.9534 .

2-(9-Phenyl-9H-xanthen-9-yl)acetonitrile (6ra)

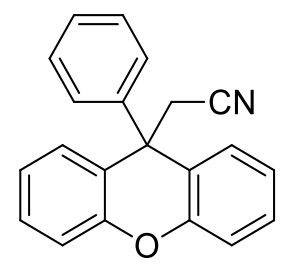

General procedure B was followed using $5 \mathbf{r}(39 \mathrm{mg}, 0.15 \mathrm{mmol})$, PivOH (31 mg, $0.30 \mathrm{mmol}, 2.0$ equiv), and $t$-BuOOt-Bu (165 $\mu \mathrm{L}, 6.00$ equiv) in acetonitrile $(1.5 \mathrm{~mL}, 0.10 \mathrm{M})$ at $125^{\circ} \mathrm{C}$ for $23 \mathrm{~h}$. Chromatography (6\% EtOAc/hexanes) afforded 6ra in $20 \%$ yield $(8.9 \mathrm{mg})$ as a colorless oil: ${ }^{1} \mathrm{H}$ $\operatorname{NMR}\left(500 \mathrm{MHz}, \mathrm{CDCl}_{3}\right) \delta 7.38(\mathrm{t}, J=7.5 \mathrm{~Hz}, 2 \mathrm{H}), 7.34-7.26(\mathrm{~m}, 5 \mathrm{H}), 7.19$ (dd, $J=8.0,1.0 \mathrm{~Hz}$, 2H), 7.01-6.97 (m, 2H), $6.84(\mathrm{dd}, J=8.0,1.5 \mathrm{~Hz}, 2 \mathrm{H}), 3.22(\mathrm{~s}, 2 \mathrm{H}) ;{ }^{13} \mathrm{C} \mathrm{NMR}\left(125 \mathrm{MHz}, \mathrm{CDCl}_{3}\right)$ $\delta 150.9,144.9,129.2,129.1,128.7,128.6,127.4,125.8,123.7,117.2,116.9,45.8,33.1 ;$ IR (film) 
3051, 3025, 2249, 1600, 1479, 1443, 1276, 1255, 753, $699 \mathrm{~cm}^{-1}$; HRMS (EI-TOF) calcd for $\mathrm{C}_{21} \mathrm{H}_{15} \mathrm{NO}[\mathrm{M}]^{+} \mathrm{m} / \mathrm{z}=297.1154$; found 297.1140.

2-(9-Phenyl-9H-fluoren-9-yl)propanenitrile (6ab)

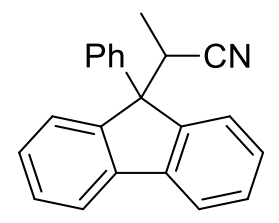

General procedure B was followed using $5 \mathbf{a}$ (36 mg, $0.15 \mathrm{mmol}$ ), PivOH (31 mg, $0.30 \mathrm{mmol}, 2.0$ equiv), and $t$-BuOOt-Bu (165 $\mu \mathrm{L}, 6.00$ equiv) in propiononitrile $2 \mathbf{b}(1.5 \mathrm{~mL}, 0.10 \mathrm{M})$ at $125{ }^{\circ} \mathrm{C}$ for $23 \mathrm{~h}$. Chromatography (4\% EtOAc/hexanes) afforded 6ab in 66\% yield (29 $\mathrm{mg}$ ) as an amorphous white solid: ${ }^{1} \mathrm{H}$ NMR $\left(500 \mathrm{MHz}, \mathrm{CDCl}_{3}\right) \delta 7.80(\mathrm{~d}, J=8.0 \mathrm{~Hz}, 1 \mathrm{H}), 7.75-7.71(\mathrm{~m}, 2 \mathrm{H})$, $7.49(\mathrm{dd}, J=7.5,1.0 \mathrm{~Hz}, 1 \mathrm{H}), 7.40(\mathrm{dd}, J=7.5,1.5 \mathrm{~Hz}, 1 \mathrm{H}), 7.35(\mathrm{dd}, J=7.5,1.5 \mathrm{~Hz}, 1 \mathrm{H}), 7.31-$ $7.18(\mathrm{~m}, 7 \mathrm{H}), 4.00(\mathrm{q}, J=7.0 \mathrm{~Hz}, 1 \mathrm{H}), 0.78(\mathrm{~d}, J=7.0 \mathrm{~Hz}, 3 \mathrm{H}) ;{ }^{13} \mathrm{C} \mathrm{NMR}\left(125 \mathrm{MHz}, \mathrm{CDCl}_{3}\right) \delta$ $148.2,146.6,141.9,141.5,140.5,128.9,128.8,128.4,128.3,128.2,127.6,126.6,125.8,123.7$, 121.9, 120.4, 120.3, 59.8, 32.9, 13.4; IR (film) 3061, 2940, 2239, 1450, 754, 732, $697 \mathrm{~cm}^{-1}$; HRMS (EI-TOF) calcd for $\mathrm{C}_{22} \mathrm{H}_{17} \mathrm{~N}[\mathrm{M}]^{+} \mathrm{m} / \mathrm{z}=295.1361$; found 295.1363 .

\section{2-(9-Phenyl-9H-fluoren-9-yl)butanenitrile (6ac)}

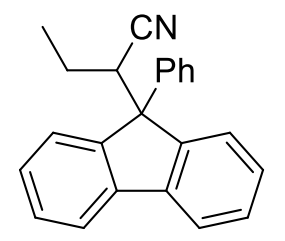

General procedure B was followed using 5a (36 mg, $0.15 \mathrm{mmol})$, PivOH (31 mg, $0.30 \mathrm{mmol}, 2.0$ equiv), and $t$-BuOOt-Bu (165 $\mu \mathrm{L}, 6.00$ equiv) in butyronitrile $2 \mathrm{c}(1.5 \mathrm{~mL}, 0.10 \mathrm{M})$ at $125^{\circ} \mathrm{C}$ for $23 \mathrm{~h}$. Chromatography (4\% EtOAc/hexanes) afforded $6 \mathbf{6 c}$ in $75 \%$ yield $(35 \mathrm{mg})$ as an amorphous white solid: ${ }^{1} \mathrm{H}$ NMR $\left(500 \mathrm{MHz}, \mathrm{CDCl}_{3}\right) \delta 7.80(\mathrm{~d}, J=8.0 \mathrm{~Hz}, 1 \mathrm{H}), 7.73(\mathrm{dd}, J=8.0,3.5 \mathrm{~Hz}, 2 \mathrm{H})$, 
$7.48(\mathrm{t}, J=7.5 \mathrm{~Hz}, 1 \mathrm{H}), 7.41-7.33(\mathrm{~m}, 2 \mathrm{H}), 7.30-7.20(\mathrm{~m}, 7 \mathrm{H}), 3.74(\mathrm{t}, J=3.5 \mathrm{~Hz}, 1 \mathrm{H}), 1.05-0.92$ $(\mathrm{m}, 5 \mathrm{H}) ;{ }^{13} \mathrm{C} \mathrm{NMR}\left(125 \mathrm{MHz}, \mathrm{CDCl}_{3}\right) \delta 148.5,147.1,141.7,140.4,128.9,128.7,128.4,128.3$ 128.2, 127.5, 126.5, 125.7, 123.7, 120.9, 120.4, 120.3, 119.8, 59.7, 41.7, 20.9, 12.4; IR (film) 3061, 2969, 2240, 1450, 747, 734, $697 \mathrm{~cm}^{-1}$; HRMS (EI-TOF) calcd for $\mathrm{C}_{23} \mathrm{H}_{19} \mathrm{~N}[\mathrm{M}]^{+} \mathrm{m} / \mathrm{z}=309.1517$; found 309.1523 .

\section{2-(9-Phenyl-9H-fluoren-9-yl)pentanenitrile (6ad)}

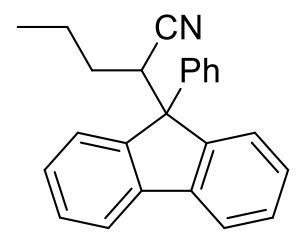

General procedure B was followed using 5a (36 mg, $0.15 \mathrm{mmol}$ ), PivOH (31 mg, $0.30 \mathrm{mmol}, 2.0$ equiv), and $t$-BuOOt-Bu (165 $\mu \mathrm{L}, 6.00$ equiv) in pentanenitrile $2 \mathbf{d}(1.5 \mathrm{~mL}, 0.10 \mathrm{M})$ at $125^{\circ} \mathrm{C}$ for $23 \mathrm{~h}$. Chromatography (5\% EtOAc/hexanes) afforded $\mathbf{6 a d}$ in $72 \%$ yield $(35 \mathrm{mg})$ as an amorphous white solid: ${ }^{1} \mathrm{H} \mathrm{NMR}\left(500 \mathrm{MHz}, \mathrm{CDCl}_{3}\right) \delta 7.80(\mathrm{~d}, J=7.5 \mathrm{~Hz}, 1 \mathrm{H}), 7.74(\mathrm{dd}, J=8.0,4.5 \mathrm{~Hz}, 2 \mathrm{H})$, $7.49(\mathrm{t}, J=7.0 \mathrm{~Hz}, 1 \mathrm{H}), 7.42-7.33(\mathrm{~m}, 2 \mathrm{H}), 7.30-7.21(\mathrm{~m}, 7 \mathrm{H}), 3.85(\mathrm{dd}, J=11.5,3.5 \mathrm{~Hz}, 1 \mathrm{H})$, $1.58-1.50(\mathrm{~m}, 1 \mathrm{H}), 1.35-1.27(\mathrm{~m}, 1 \mathrm{H}), 1.09-0.98(\mathrm{~m}, 1 \mathrm{H}), 0.87-0.80(\mathrm{~m}, 1 \mathrm{H}), 0.75(\mathrm{t}, J=7.5 \mathrm{~Hz}$ $3 \mathrm{H}) ;{ }^{13} \mathrm{C}$ NMR $\left(125 \mathrm{MHz}, \mathrm{CDCl}_{3}\right) \delta 148.4,147.1,141.7,141.6,140.4,128.9,128.7,128.4,128.3$, 128.1, 127.5, 126.6, 125.7, 123.7, 121.2, 120.4, 120.3, 59.6, 39.4, 29.2, 21.0, 13.5; IR (film) 3060, 2960, 2245, 1450, 755, 733, $697 \mathrm{~cm}^{-1}$; HRMS (EI-TOF) calcd for $\mathrm{C}_{24} \mathrm{H}_{21} \mathrm{~N}[\mathrm{M}]^{+} \mathrm{m} / \mathrm{z}=323.1674$ found 323.1685 .

2-Methoxy-2-(9-phenyl-9H-fluoren-9-yl)acetonitrile (6ae)<smiles>COC(C#N)C1(c2ccccc2)c2ccccc2-c2ccccc21</smiles> 
General procedure B was followed using 5a (36 mg, $0.15 \mathrm{mmol})$, PivOH (31 mg, $0.30 \mathrm{mmol}, 2.0$ equiv), and $t$-BuOOt-Bu (165 $\mu \mathrm{L}, 6.00$ equiv) in 2-methoxyacetonitrile $2 \mathrm{e}(1.5 \mathrm{~mL}, 0.10 \mathrm{M})$ at 125 ${ }^{\circ} \mathrm{C}$ for $23 \mathrm{~h}$. Chromatography (6\% EtOAc/hexanes) afforded 6ae in $42 \%$ yield $(20 \mathrm{mg}$ ) as an amorphous white solid: ${ }^{1} \mathrm{H}$ NMR $\left(500 \mathrm{MHz}, \mathrm{CDCl}_{3}\right) \delta 7.82(\mathrm{~d}, J=7.6 \mathrm{~Hz}, 1 \mathrm{H}), 7.80(\mathrm{~d}, J=7.6$ $\mathrm{Hz}, 1 \mathrm{H}), 7.57(\mathrm{~d}, J=7.5 \mathrm{~Hz}, 1 \mathrm{H}), 7.47(\mathrm{td}, J=7.0,1.0 \mathrm{~Hz}, 1 \mathrm{H}), 7.42(\mathrm{td}, J=7.0,1.0 \mathrm{~Hz}, 1 \mathrm{H})$, $7.37(\mathrm{~d}, J=7.5 \mathrm{~Hz}, 1 \mathrm{H}), 7.33(\mathrm{td}, J=8.0,1.0 \mathrm{~Hz}, 1 \mathrm{H}), 7.29-7.20(\mathrm{~m}, 6 \mathrm{H}), 4.80(\mathrm{~s}, 1 \mathrm{H}), 3.55(\mathrm{~s}$, $3 \mathrm{H}) ;{ }^{13} \mathrm{C} \mathrm{NMR}\left(125 \mathrm{MHz}, \mathrm{CDCl}_{3}\right) \delta 146.9,146.4,141.4,140.8,139.8,129.0,128.9,128.8,128.0$, 127.9, 127.5, 127.1, 126.9, 125.5, 120.6, 120.4, 116.5, 76.5, 61.1, 59.1; IR (film) 3061, 2930, 2250, 1450, 1099, 756, $736 \mathrm{~cm}^{-1}$; HRMS (EI-TOF) calcd for $\mathrm{C}_{22} \mathrm{H}_{17} \mathrm{NO}[\mathrm{M}]^{+} \mathrm{m} / \mathrm{z}=311.1310$; found 311.1310 (masses identical).

\section{2-Methyl-2-(9-phenyl-9H-fluoren-9-yl)propanenitrile (6af)}

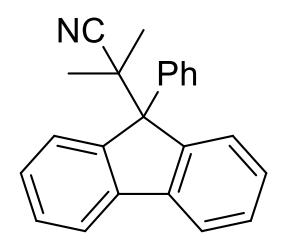

General procedure B was followed using 5a (36 mg, $0.15 \mathrm{mmol})$, PivOH (31 mg, $0.30 \mathrm{mmol}, 2.0$ equiv), and $t$-BuOOt-Bu (165 $\mu \mathrm{L}, 6.00$ equiv) in isobutyronitrile $2 \mathbf{f}(1.5 \mathrm{~mL}, 0.10 \mathrm{M})$ at $125^{\circ} \mathrm{C}$ for $23 \mathrm{~h}$. Chromatography (4\% EtOAc/hexanes) afforded 6af in $42 \%$ yield (20 $\mathrm{mg}$ ) as a colorless oil: ${ }^{1} \mathrm{H} \mathrm{NMR}\left(500 \mathrm{MHz}, \mathrm{CDCl}_{3}\right) \delta 7.76(\mathrm{~d}, J=7.5 \mathrm{~Hz}, 2 \mathrm{H}), 7.59-7.55(\mathrm{~m}, 4 \mathrm{H}), 7.42(\mathrm{t}, J=7.5 \mathrm{~Hz}$, 2H), 7.32-7.20 (m, 5H), 1.27 (s, 6H); ${ }^{13} \mathrm{C}$ NMR (125 MHz, $\left.\mathrm{CDCl}_{3}\right) \delta$ 147.9, 141.4, 140.7, 128.6, 128.5, 128.1, 127.7, 127.1, 126.5, 126.4, 120.1, 63.0, 38.2, 24.7; IR (film) 3060, 2229, 1448, 744, $728 \mathrm{~cm}^{-1}$; HRMS (EI-TOF) calcd for $\mathrm{C}_{23} \mathrm{H}_{19} \mathrm{~N}[\mathrm{M}]^{+} \mathrm{m} / \mathrm{z}=309.1517$; found 309.1529.

\section{1-(9-Phenyl-9H-fluoren-9-yl)cyclohexanecarbonitrile (6ag)}




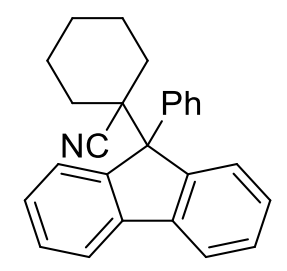

General procedure B was followed using 5a (36 mg, $0.15 \mathrm{mmol})$, PivOH (31 mg, $0.30 \mathrm{mmol}, 2.0$ equiv), and $t$-BuOOt-Bu (165 $\mu \mathrm{L}, 6.00$ equiv) in cyclohexanecarbonitrile $2 \mathrm{~g}(1.5 \mathrm{~mL}, 0.10 \mathrm{M})$ at $125^{\circ} \mathrm{C}$ for $23 \mathrm{~h}$. Chromatography (4\% EtOAc/hexanes) afforded 6ag in $61 \%$ yield $(32 \mathrm{mg}$ ) as an amorphous white solid: ${ }^{1} \mathrm{H} \mathrm{NMR}\left(500 \mathrm{MHz}, \mathrm{CDCl}_{3}\right) \delta 7.75(\mathrm{~d}, J=7.5 \mathrm{~Hz}, 2 \mathrm{H}), 7.61-7.58(\mathrm{~m}, 4 \mathrm{H})$, $7.41(\mathrm{t}, J=7.5 \mathrm{~Hz}, 2 \mathrm{H}), 7.30(\mathrm{td}, J=7.5,1.0 \mathrm{~Hz}, 2 \mathrm{H}), 7.27-7.18(\mathrm{~m}, 3 \mathrm{H}), 1.85-1.39(\mathrm{~m}, 7 \mathrm{H}), 1.30-$ $1.25(\mathrm{~m}, 2 \mathrm{H}), 0.97-0.88(\mathrm{~m}, 1 \mathrm{H}) ;{ }^{13} \mathrm{C} \mathrm{NMR}\left(125 \mathrm{MHz}, \mathrm{CDCl}_{3}\right) \delta$ 148.1, 141.6, 140.4, 128.6, 128.4, 128.3, 127.6, 127.1, 126.9, 123.7, 119.9, 63.7, 45.9, 31.3, 25.1, 22.9; IR (film) 3058, 2937, 2227, 1449, 743, $699 \mathrm{~cm}^{-1}$; HRMS (EI-TOF) calcd for $\mathrm{C}_{26} \mathrm{H}_{23} \mathrm{~N}[\mathrm{M}]^{+} \mathrm{m} / \mathrm{z}=349.1830$; found 349.1831 .

\subsection{Derivatization of Product 3aa}

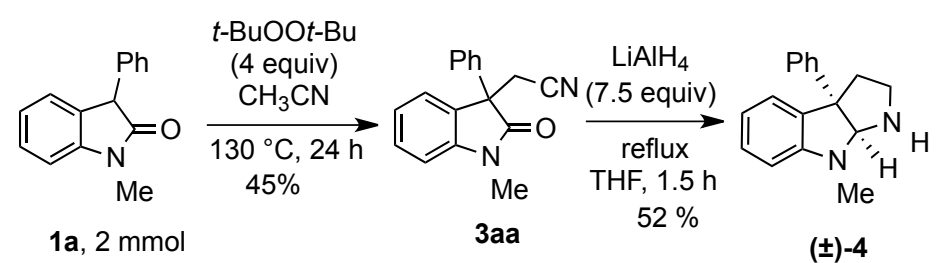

Large scale cyanomethylation and derivatization to 8 -methyl-3a-phenyl-1,2,3,3a,8,8ahexahydropyrrolo[2,3-b]indole (4). Oxindole 1a (446 mg, $2.00 \mathrm{mmol}$ ) was submitted to the General procedure A. Purification by chromatography (100:12 hexane/EtOAc) afforded compound 3aa in $45 \%$ yield $(236 \mathrm{mg})$ as a brown solid. To a precooled $\left(5^{\circ} \mathrm{C}\right)$ stirred suspension of $\mathrm{LiAlH}_{4}(256 \mathrm{mg}, 6.75 \mathrm{mmol})$ in anhydrous THF $(10 \mathrm{~mL})$ and under argon atmosphere was added the oxindole 3aa $(236 \mathrm{mg}, 0.900 \mathrm{mmol})$ in THF $(10 \mathrm{~mL})$, and the mixture was heated at 
reflux for $1.5 \mathrm{~h}$. After cooling, the reaction mixture in an ice/water bath, the reaction was quenched by adding, in sequence, EtOAc $(10 \mathrm{~mL})$ and water $(5 \mathrm{~mL})$. The solids were removed by filtration and washed with EtOAc $(2 \times 10 \mathrm{~mL})$. The combined organic phases were washed with brine, dried, and concentrated under reduced pressure. The resulting pyrroloindoline was purified by chromatography on silica gel (100:1 $\left.\mathrm{CH}_{2} \mathrm{Cl}_{2} / \mathrm{MeOH}\right)$ affording compound 4 in 52\% yield (117 mg) as a colorless oil: ${ }^{1} \mathrm{H}$ NMR $\left(500 \mathrm{MHz}, \mathrm{CDCl}_{3}\right) \delta$ 7.35-7.28 (m, 4H), $7.20(\mathrm{t}, J=7.0 \mathrm{~Hz}, 1 \mathrm{H}), 7.09$ (t, $J=7.5 \mathrm{~Hz}, 1 \mathrm{H}), 6.89(\mathrm{~d}, J=7.0 \mathrm{~Hz}, 1 \mathrm{H}), 6.60(\mathrm{t}, J=7.5 \mathrm{~Hz}, 1 \mathrm{H}), 6.39(\mathrm{~d}, J=8.0 \mathrm{~Hz}, 1 \mathrm{H}), 4.94$ (s, 1H), 3.24-3.19 (m, 1H), $2.91(\mathrm{~s}, 3 \mathrm{H}), 2.79-2.72(\mathrm{~m}, 1 \mathrm{H}), 2.53-2.47(\mathrm{~m}, 1 \mathrm{H}), 2.42-2.37(\mathrm{~m}, 1 \mathrm{H})$, $2.03(\mathrm{~s}, 1 \mathrm{H}) ;{ }^{13} \mathrm{C} \mathrm{NMR}\left(125 \mathrm{MHz}, \mathrm{CDCl}_{3}\right) \delta 151.3,146.2,134.2,128.4,128.1,126.3,126.2,124.2$, 116.8, 105.1, 92.9, 61.3, 46.8, 41.8, 31.3; IR (film) 2933, 2875, 1603, 1492, 1296, 738, $699 \mathrm{~cm}^{-1}$; HRMS (EI-TOF) calcd for $\mathrm{C}_{17} \mathrm{H}_{18} \mathrm{~N}_{2}[\mathrm{M}]^{+} \mathrm{m} / \mathrm{z}=250.1470$; found 250.1482 .

\section{References}

(1) Heaney, H.; Cardona, F.; Goti, A.; Frederick, A. L. 2013. Hydrogen Peroxide-Urea. e-EROS Encyclopedia of Reagents for Organic Synthesis.

(2) (a) Trost, B. M.; Masters, J. T.; Burns, A. C. Palladium-Catalyzed Asymmetric Allylic Alkylation of 3-Aryloxindoles with Allylidene Dipivalate: A Useful Enol Pivalate Product. Angew. Chem., Int. Ed. 2013, 52, 2260. (b) Trost, B. M.; Xie, J.; Sieber, J. D. The Palladium Catalyzed Asymmetric Addition of Oxindoles and Allenes: An Atom-Economical Versatile Method for the Construction of Chiral Indole Alkaloids. J. Am. Chem. Soc. 2011, 133, 20611.

(3) (a) Shen, X.; Gu, N. N.; Liu, P.; Mao, X. W.; Xie, J. W.; Liu, Y.; Hen, L.; Dai, B. A Simple and Efficient Synthesis of 9-Arylfluorenes via Metal-Free Reductive Coupling of Arylboronic Acids and N-Tosylhydrazones in situ. RSC $A d v$. 2015, 5, 63726. (b) Vougioukalakis, G. C.; Roubelakis, M. M.; Orfanopoulos, M. Radical Reactivity of Aza[60]fullerene: Preparation of Monoadducts and Limitations. J. Org. Chem. 2010, 75, 4124.

(4) Sazepin, C. C.; Wang, Q.; Sammis, G. M.; Zhu, J. P. Copper-Catalyzed Intermolecular Carboetherification of Unactivated Alkenes by Alkyl Nitriles and Alcohols. Angew. Chem. Int. Ed. 2015, 54, 5443.

(5) Frenette, M.; Aliaga, C.; Font-Sanchis, E.; Scaiano, J. C. Bond Dissociation Energies for Radical- Dimers Derived from Highly Stabilized Carbon-Centered Radicals Org. Lett. 2004, 6, 2579-2582.

(6) Hong, G.; Nahide, P. D.; Neelam, U. K.; Amadeo, P.; Vijeta, A.; Curto, J. M.; Hendrick, C. E.; VanGelder, K. F.; Kozlowski, M. C. Palladium-Catalyzed Chemoselective Activation of $\mathrm{sp}^{3}$ vs sp ${ }^{2} \mathrm{C}-\mathrm{H}$ Bonds: Oxidative Coupling To Form Quaternary Centers ACS Catal. 2019, 9, 3716 . 


\section{Copies of ${ }^{1} \mathrm{H}$ NMR, ${ }^{13} \mathrm{C}$ NMR and ${ }^{19} \mathrm{~F}$ NMR}

3aa ${ }^{1} \mathrm{H}$ NMR (500 MHz, $\left.\mathrm{CDCl}_{3}\right),{ }^{13} \mathrm{C}$ NMR $\left(125 \mathrm{MHz}, \mathrm{CDCl}_{3}\right)$

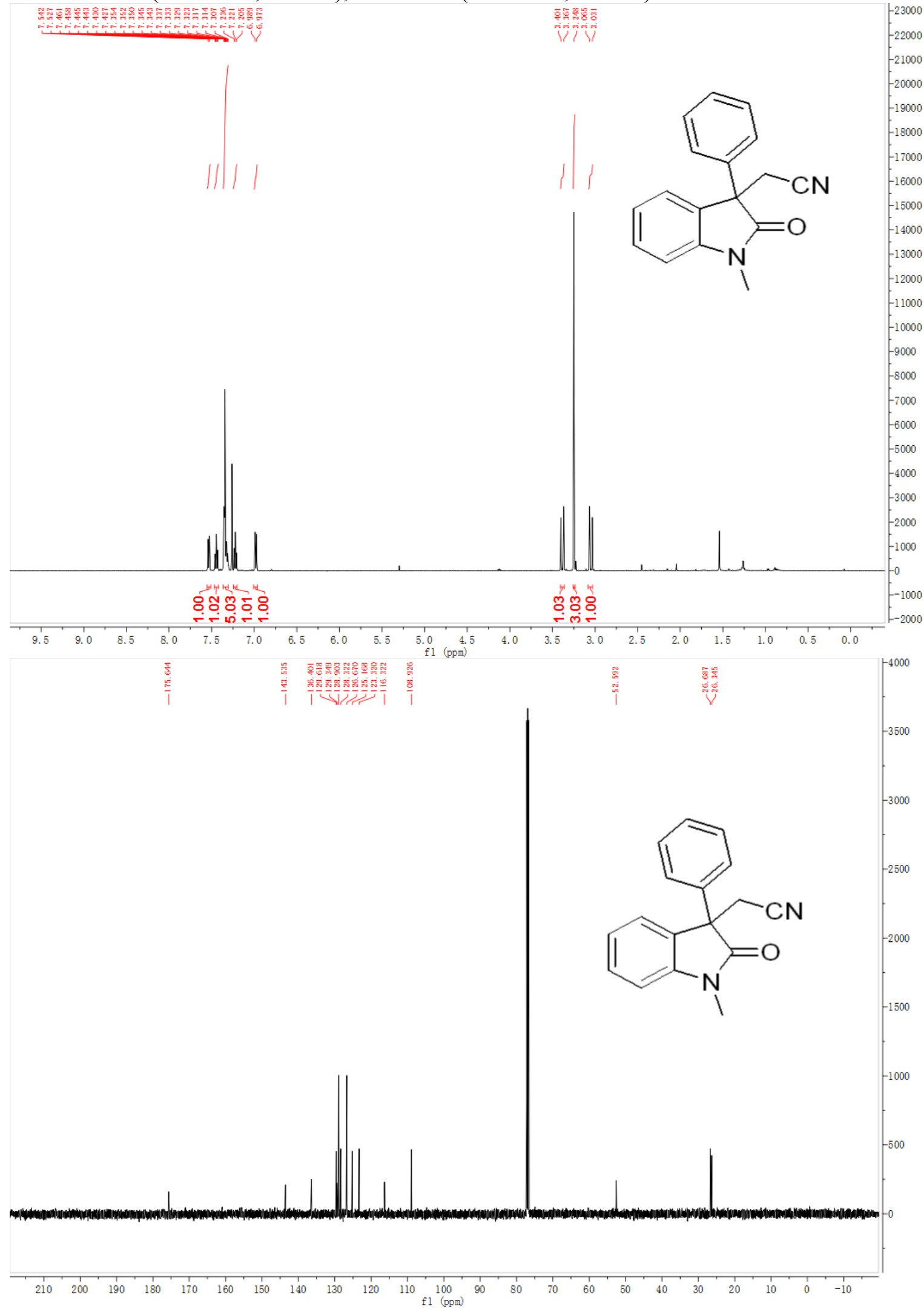


3ba ${ }^{1} \mathrm{H}$ NMR $\left(500 \mathrm{MHz}, \mathrm{CDCl}_{3}\right),{ }^{13} \mathrm{C} \mathrm{NMR}\left(125 \mathrm{MHz}, \mathrm{CDCl}_{3}\right)$

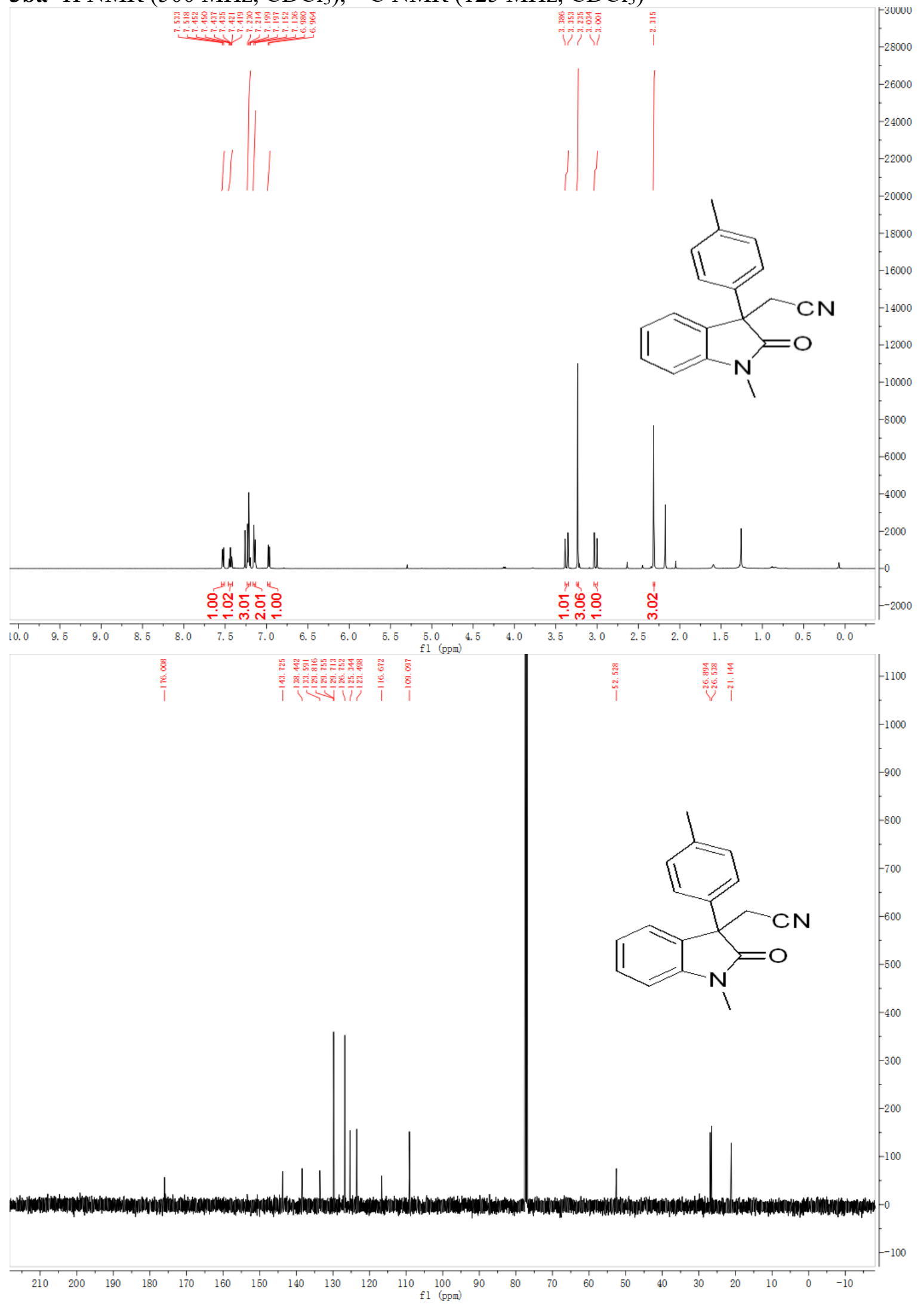


3ca ${ }^{1} \mathrm{H}$ NMR (500 MHz, $\left.\mathrm{CDCl}_{3}\right),{ }^{13} \mathrm{C} \mathrm{NMR}\left(125 \mathrm{MHz}, \mathrm{CDCl}_{3}\right)$

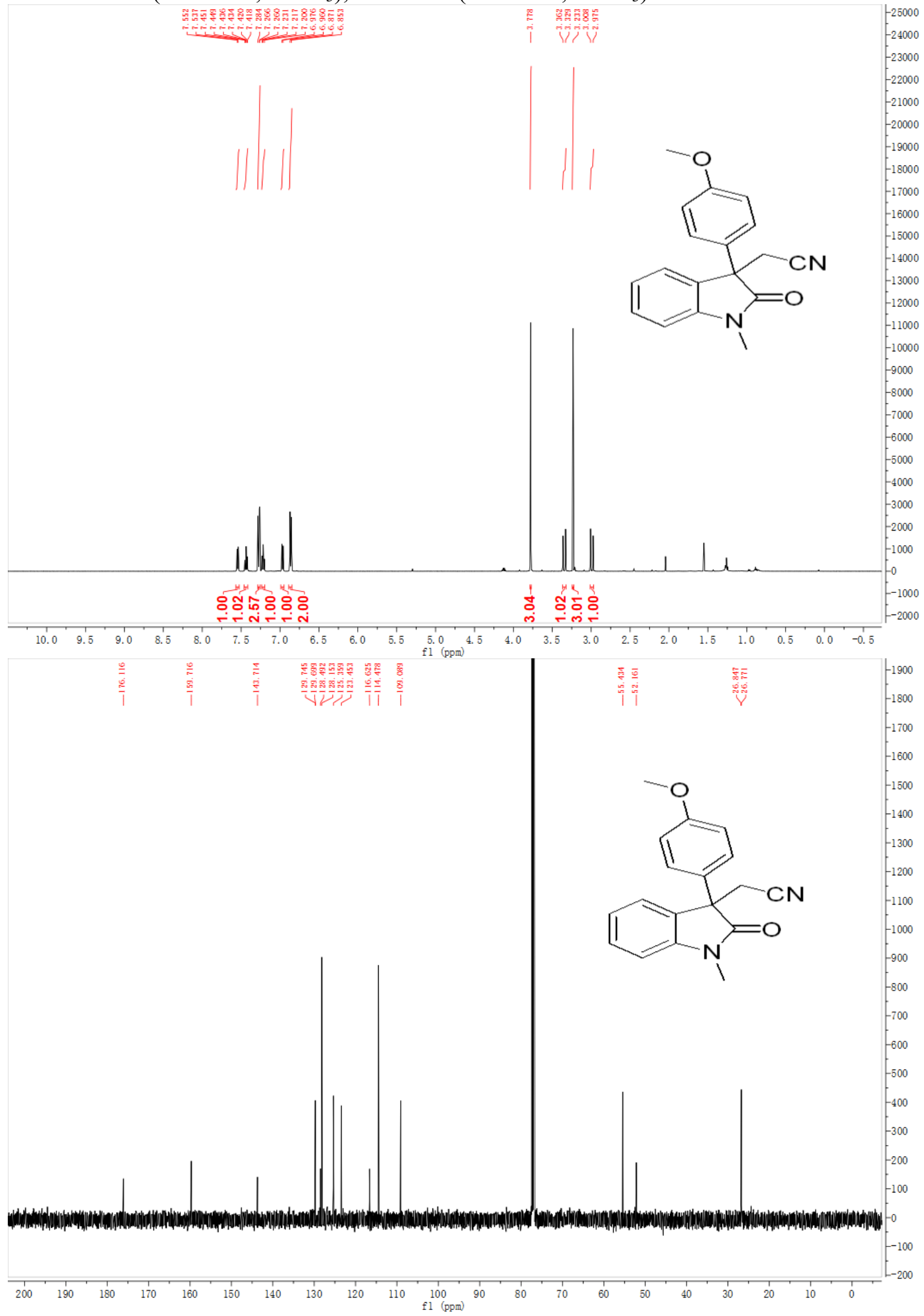


3da ${ }^{1} \mathrm{H} \mathrm{NMR}\left(500 \mathrm{MHz}, \mathrm{CDCl}_{3}\right),{ }^{13} \mathrm{C} \mathrm{NMR}\left(125 \mathrm{MHz}, \mathrm{CDCl}_{3}\right),{ }^{19} \mathrm{~F} \mathrm{NMR}\left(376 \mathrm{MHz}, \mathrm{CDCl}_{3}\right)$

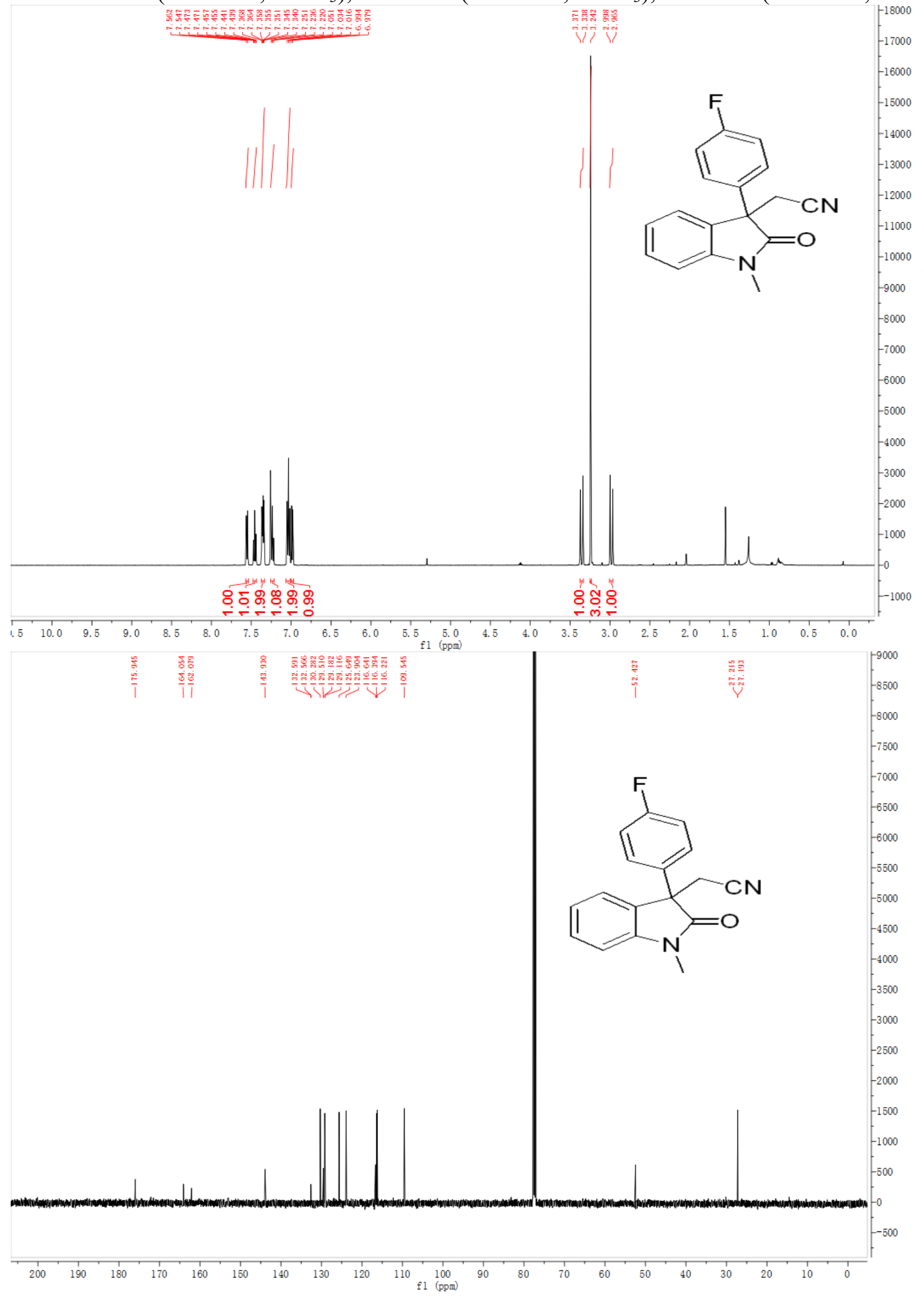




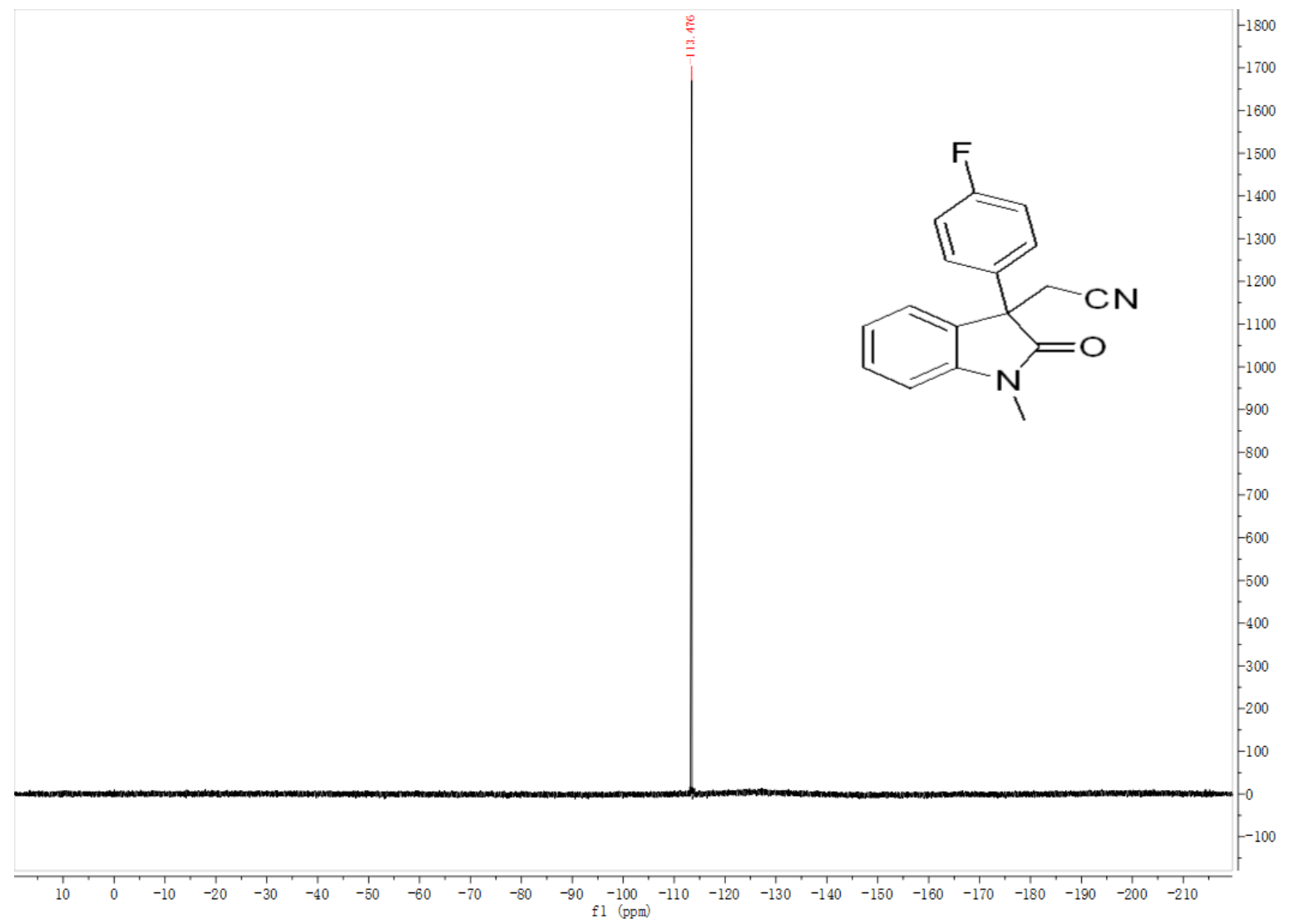


3ea ${ }^{1} \mathrm{H}$ NMR $\left(500 \mathrm{MHz}, \mathrm{CDCl}_{3}\right),{ }^{13} \mathrm{C} \mathrm{NMR}\left(125 \mathrm{MHz}, \mathrm{CDCl}_{3}\right)$

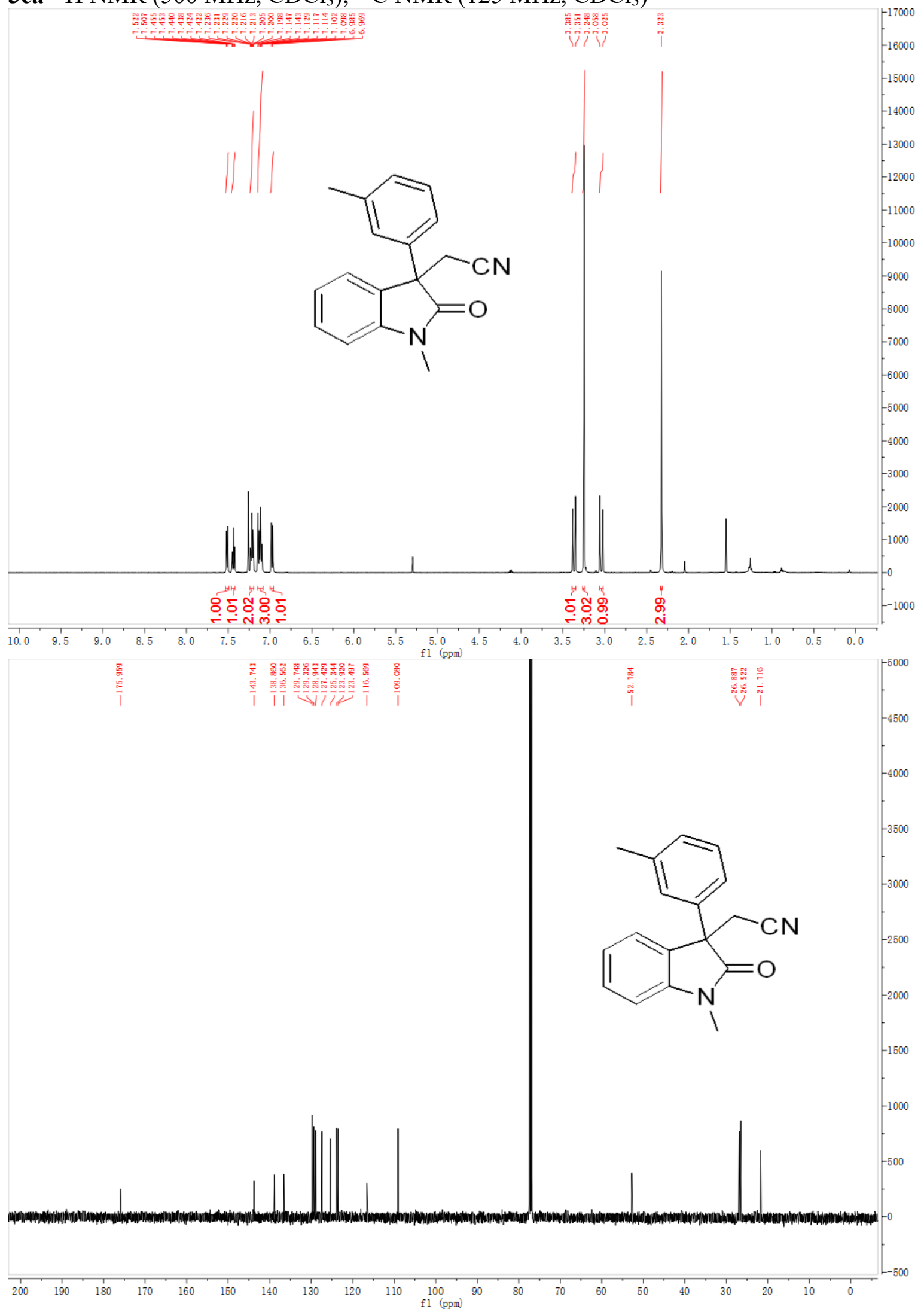


3fa ${ }^{1} \mathrm{H}$ NMR (500 MHz, $\left.\mathrm{CDCl}_{3}\right),{ }^{13} \mathrm{C}$ NMR (125 MHz, $\left.\mathrm{CDCl}_{3}\right)$

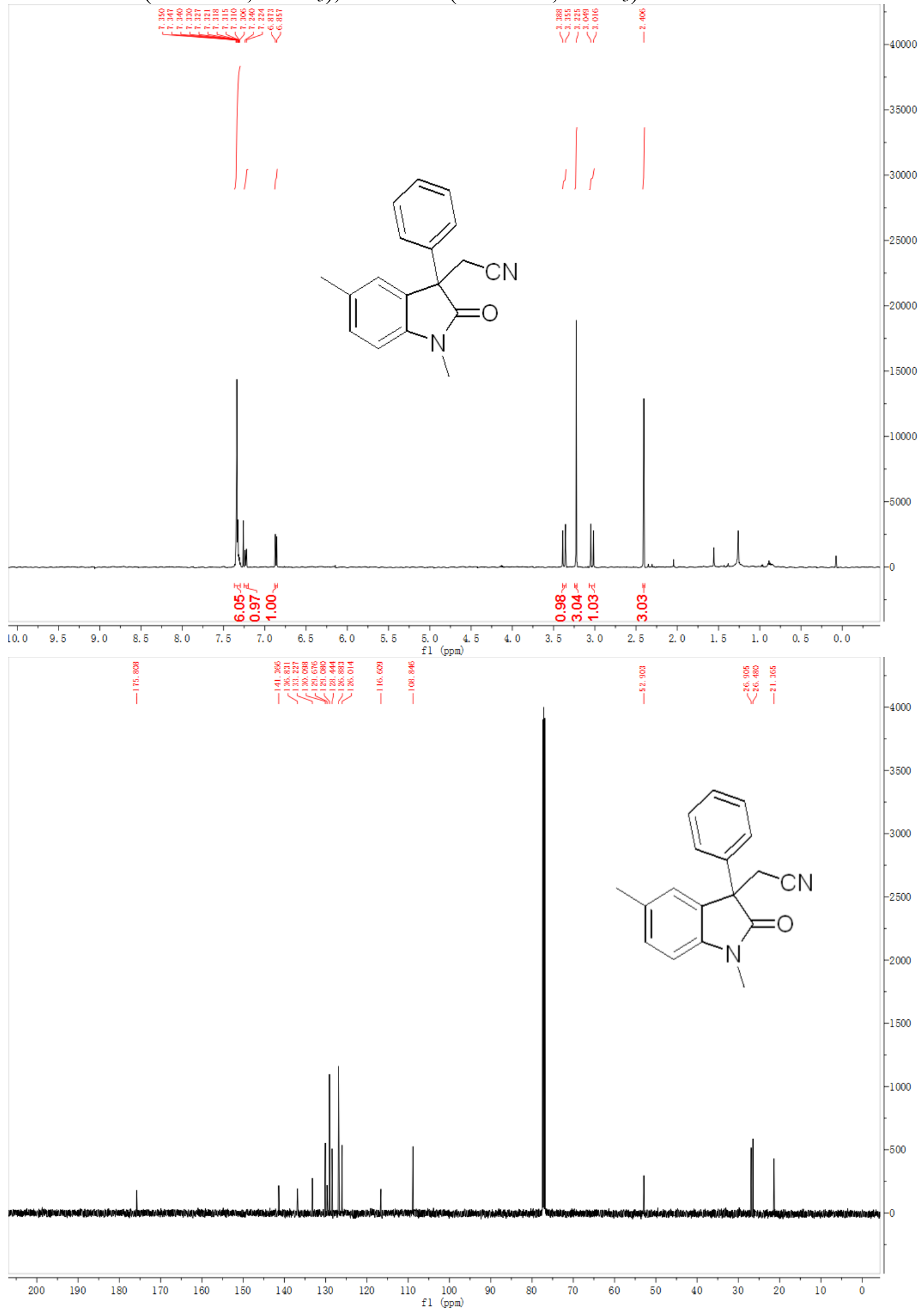


3ga ${ }^{1} \mathrm{H}$ NMR $\left(500 \mathrm{MHz}, \mathrm{CDCl}_{3}\right),{ }^{13} \mathrm{C} \mathrm{NMR}\left(125 \mathrm{MHz}, \mathrm{CDCl}_{3}\right)$

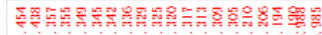

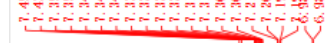

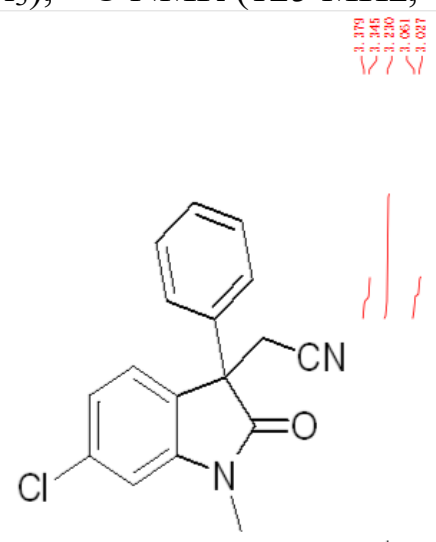

जi:
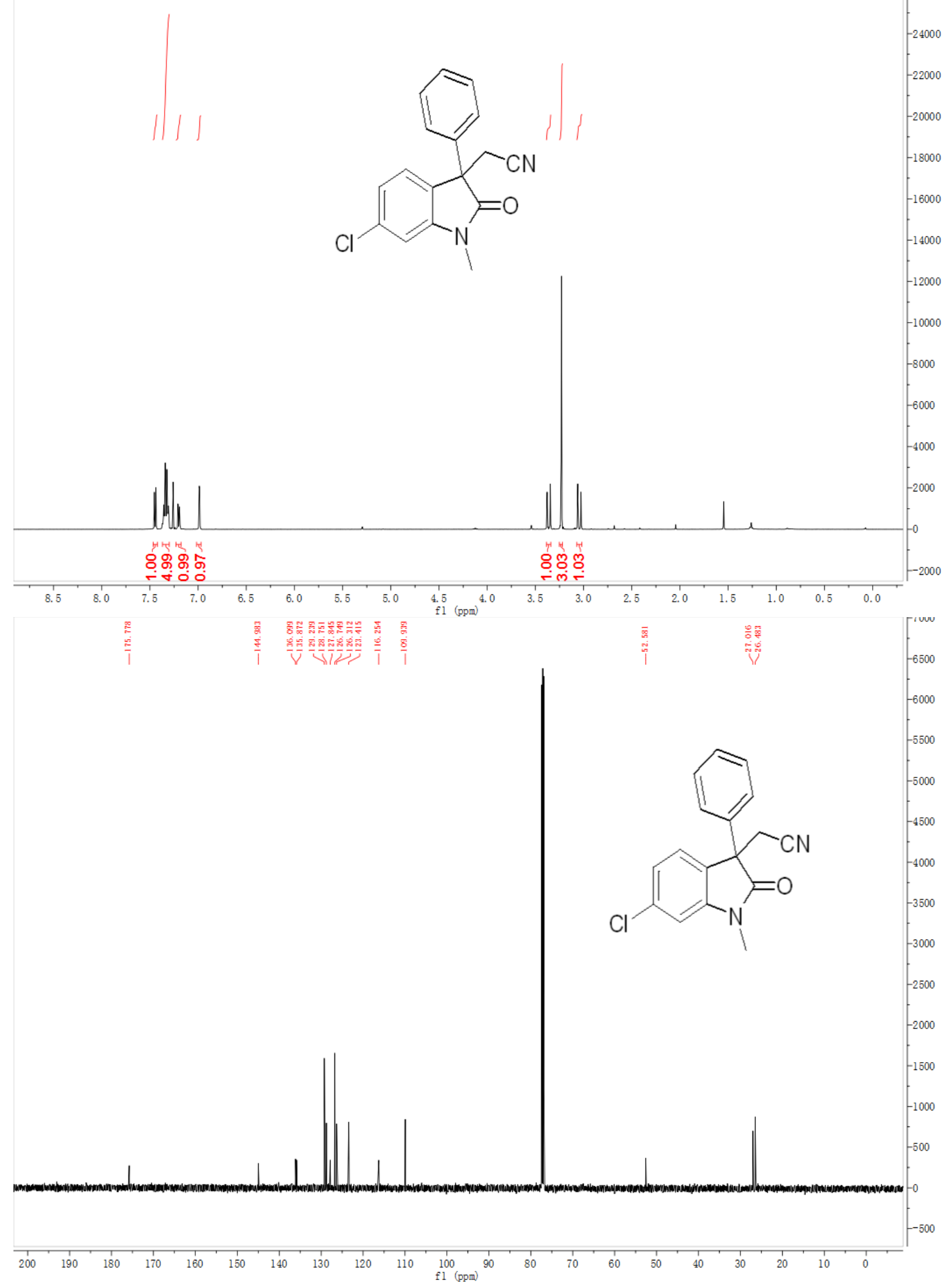
3ha ${ }^{1} \mathrm{H}$ NMR (500 MHz, $\left.\mathrm{CDCl}_{3}\right),{ }^{13} \mathrm{C}$ NMR $\left(125 \mathrm{MHz}, \mathrm{CDCl}_{3}\right)$

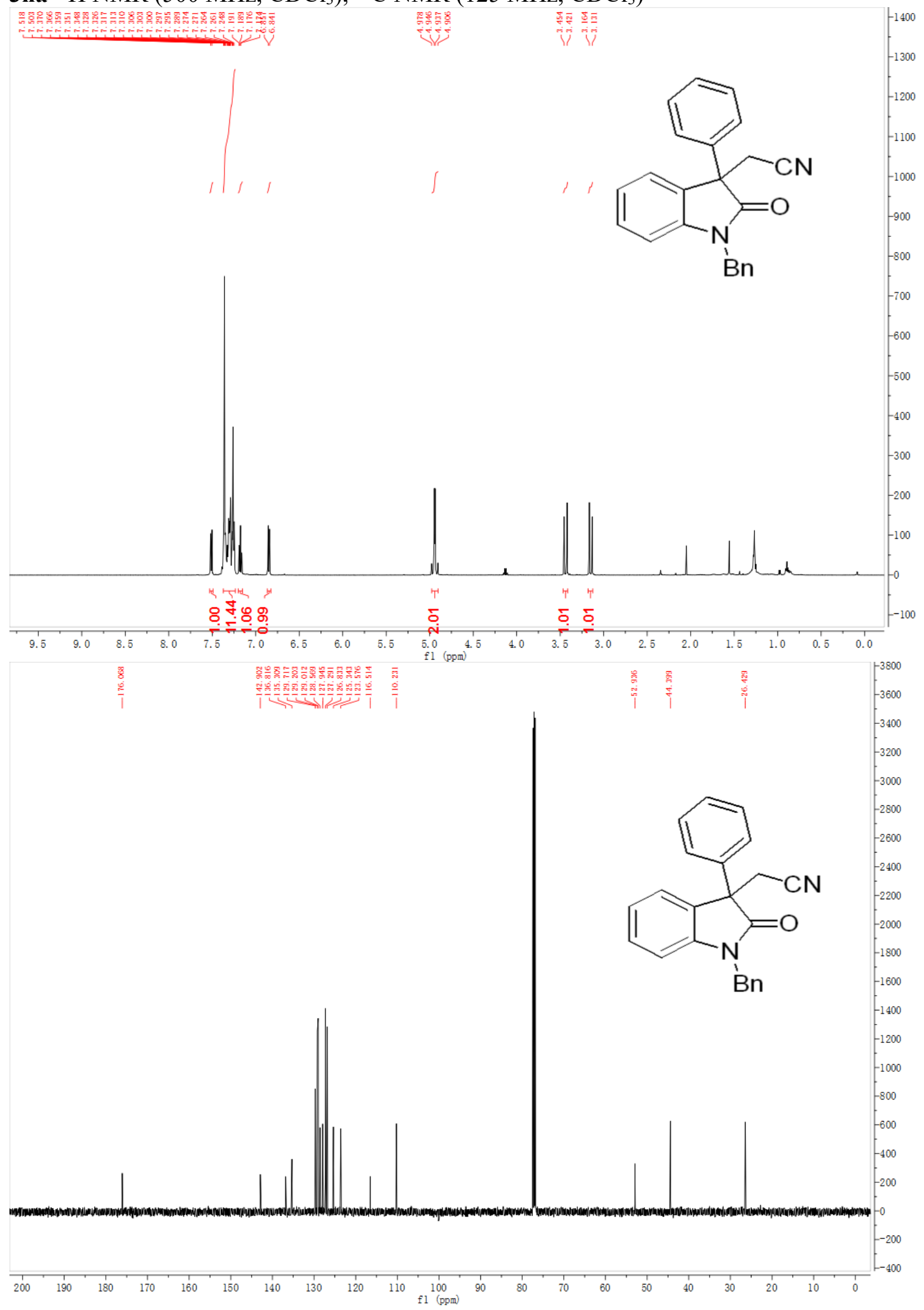


6aa ${ }^{1} \mathrm{H}$ NMR $\left(500 \mathrm{MHz}, \mathrm{CDCl}_{3}\right),{ }^{13} \mathrm{C} \mathrm{NMR}\left(125 \mathrm{MHz}, \mathrm{CDCl}_{3}\right)$

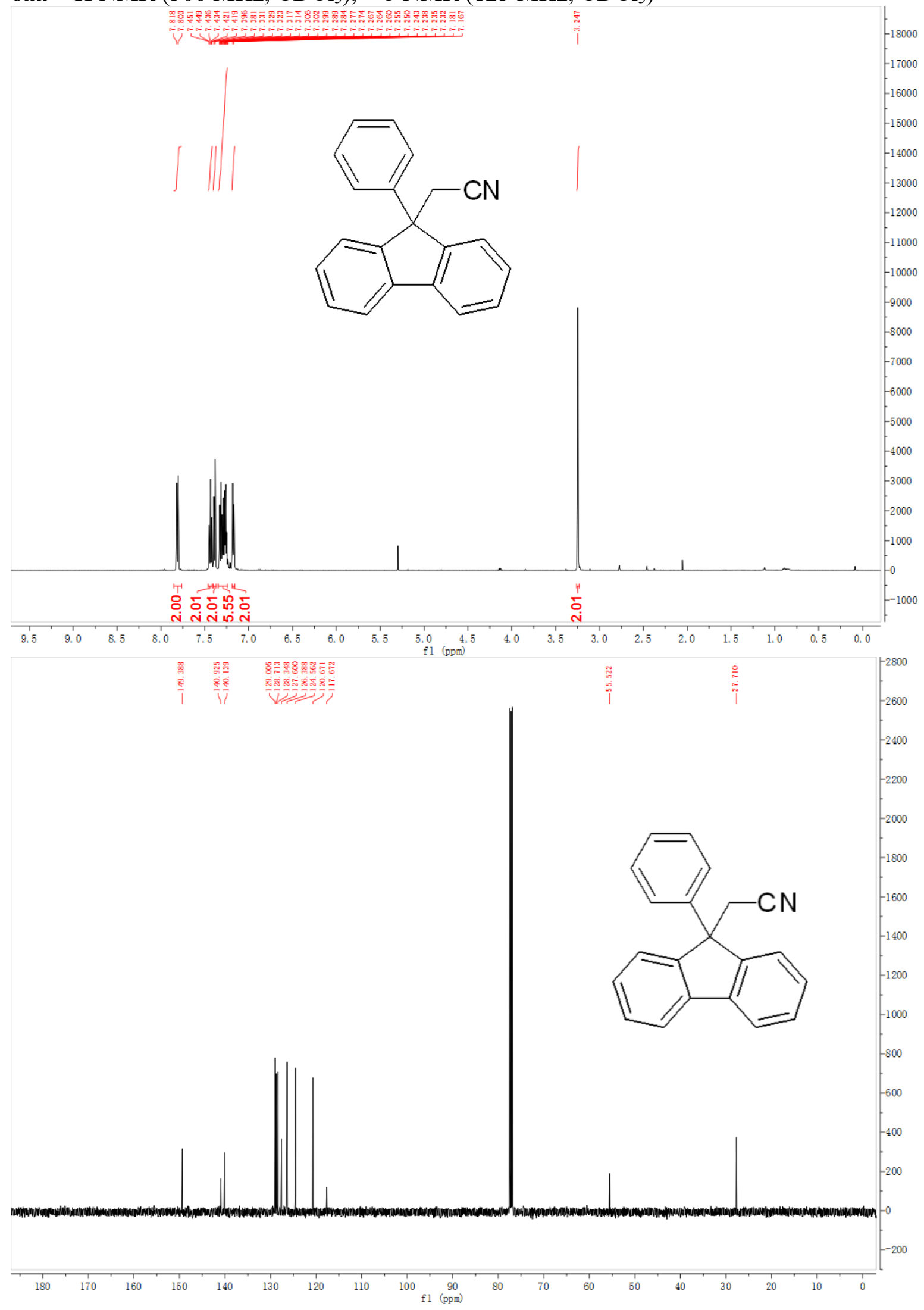


6ba ${ }^{1} \mathrm{H}$ NMR (500 MHz, $\left.\mathrm{CDCl}_{3}\right),{ }^{13} \mathrm{C} \mathrm{NMR}\left(125 \mathrm{MHz}, \mathrm{CDCl}_{3}\right)$

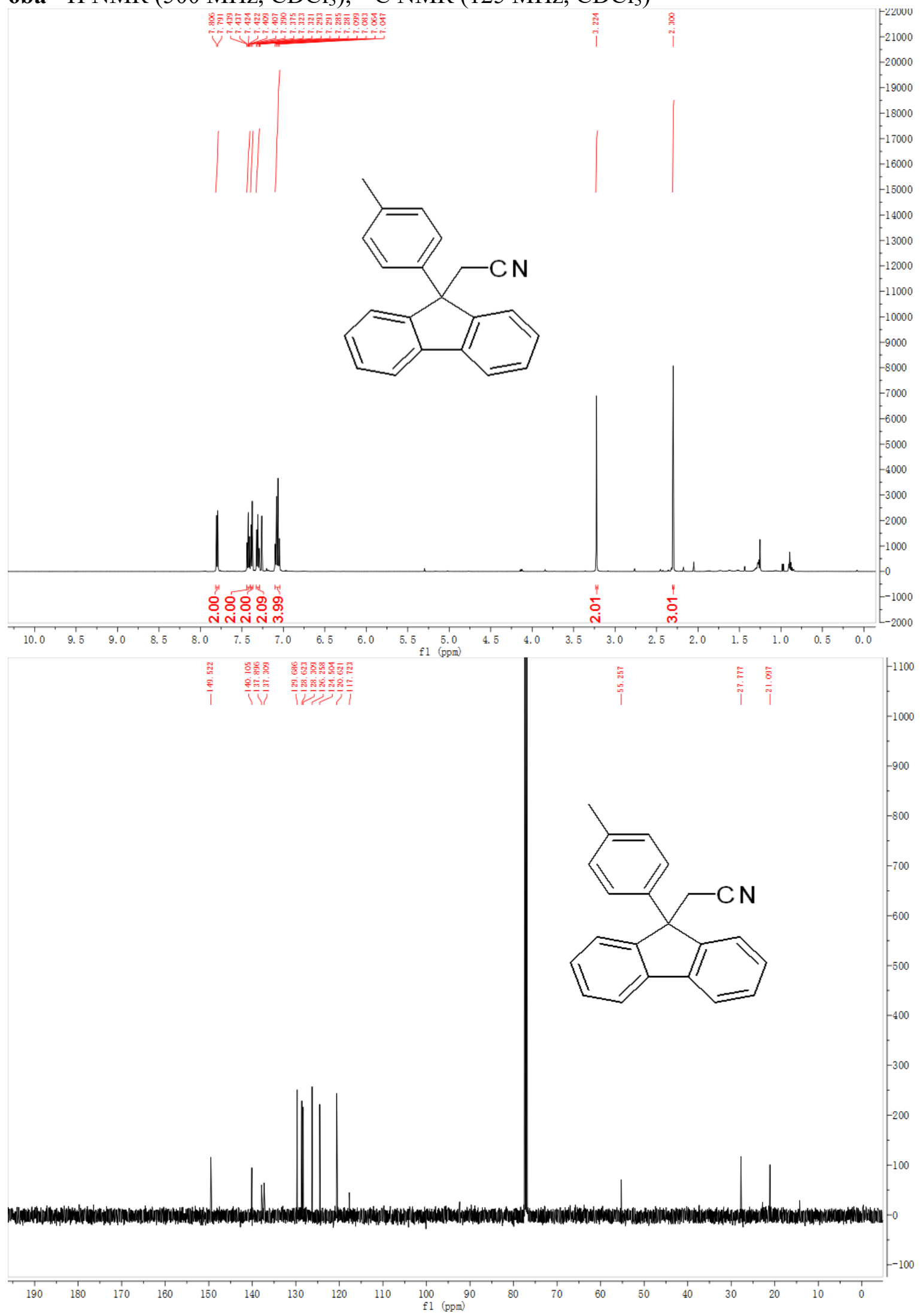


6ca ${ }^{1} \mathrm{H}$ NMR (500 MHz, $\left.\mathrm{CDCl}_{3}\right),{ }^{13} \mathrm{C}$ NMR (125 MHz, $\left.\mathrm{CDCl}_{3}\right)$

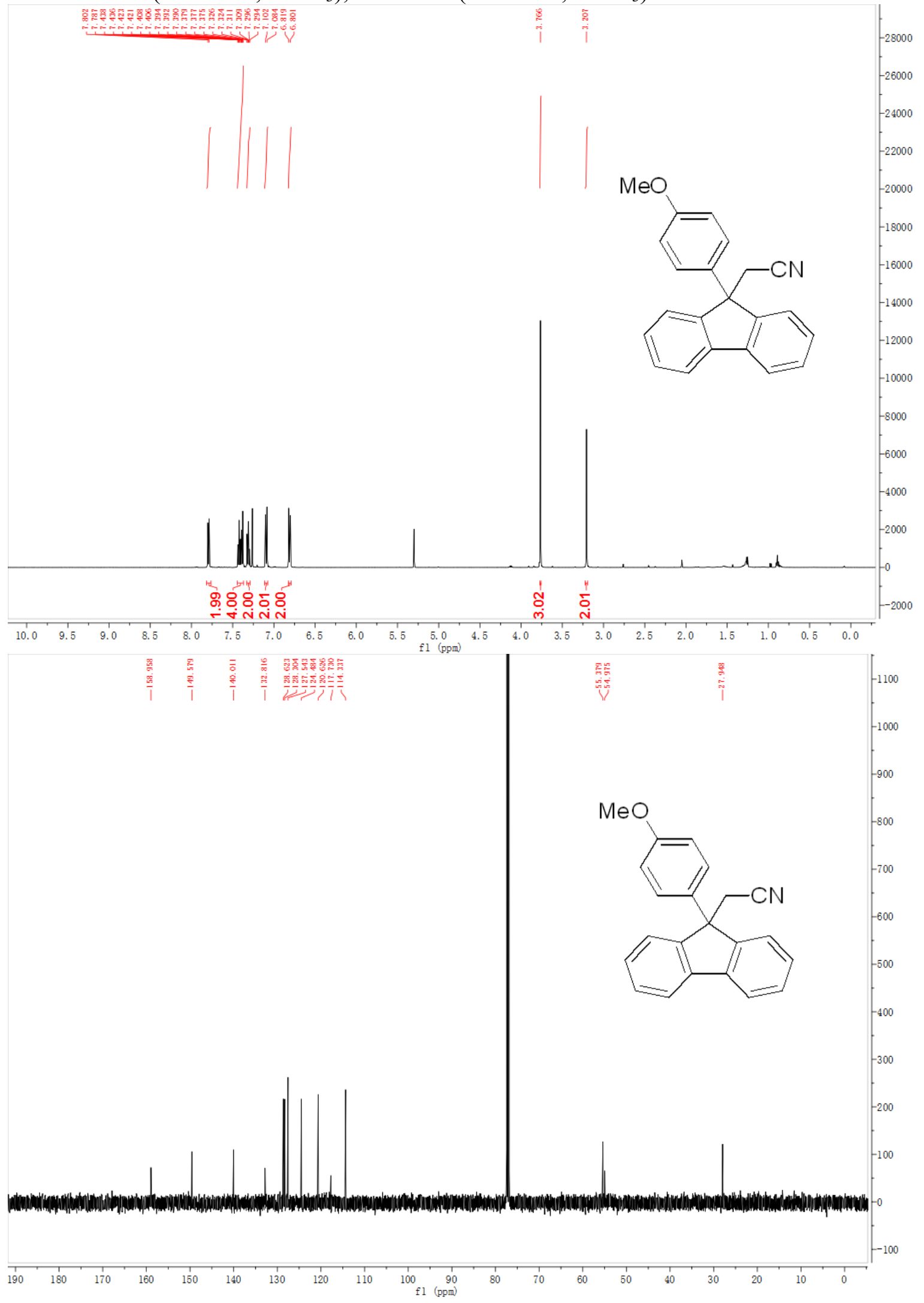


6da ${ }^{1} \mathrm{H}$ NMR $\left(500 \mathrm{MHz}, \mathrm{CDCl}_{3}\right),{ }^{13} \mathrm{C} \mathrm{NMR}\left(125 \mathrm{MHz}, \mathrm{CDCl}_{3}\right)$

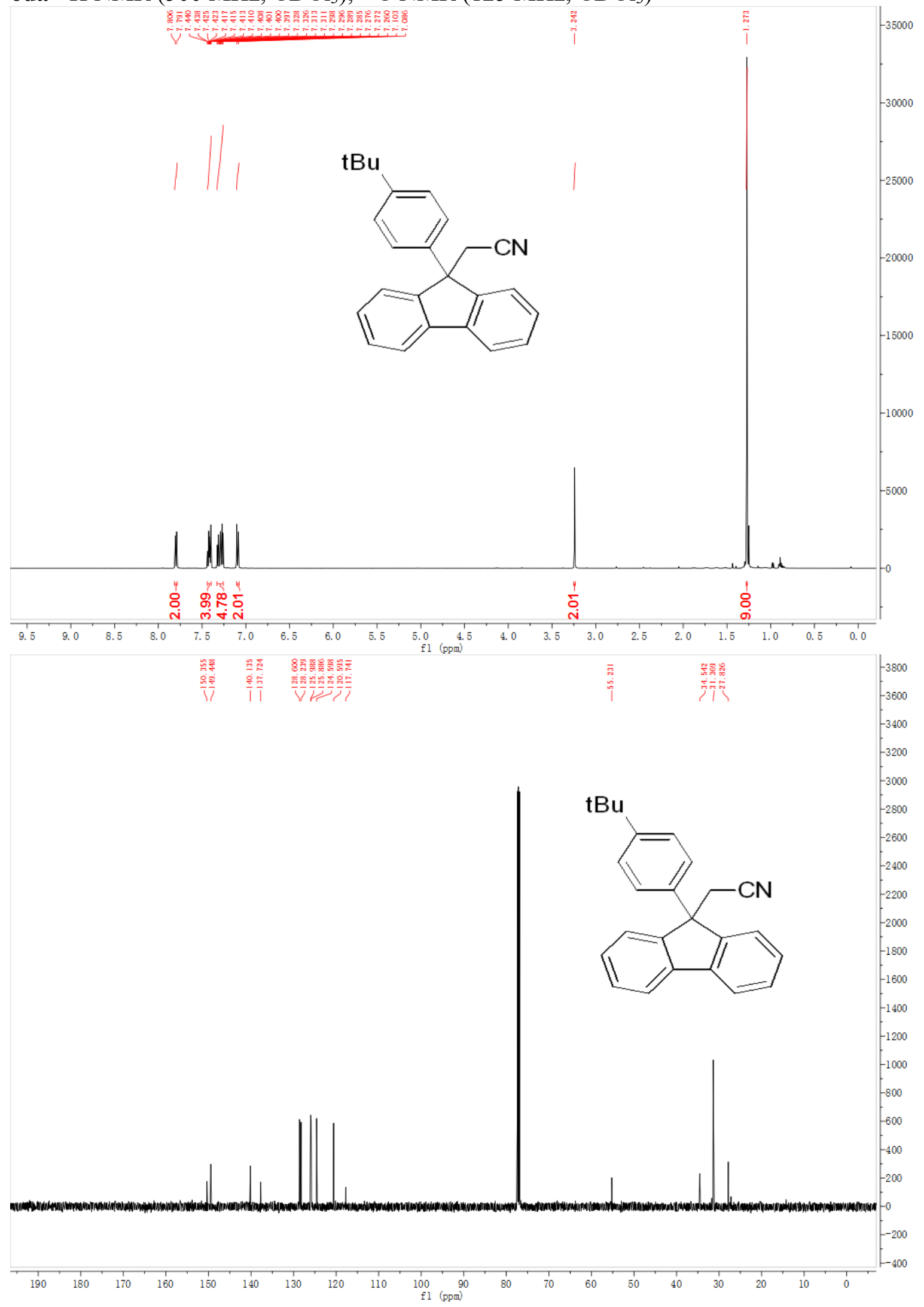


6ea ${ }^{1} \mathrm{H}$ NMR (500 MHz, $\left.\mathrm{CDCl}_{3}\right),{ }^{13} \mathrm{C} \mathrm{NMR}\left(125 \mathrm{MHz}, \mathrm{CDCl}_{3}\right)$

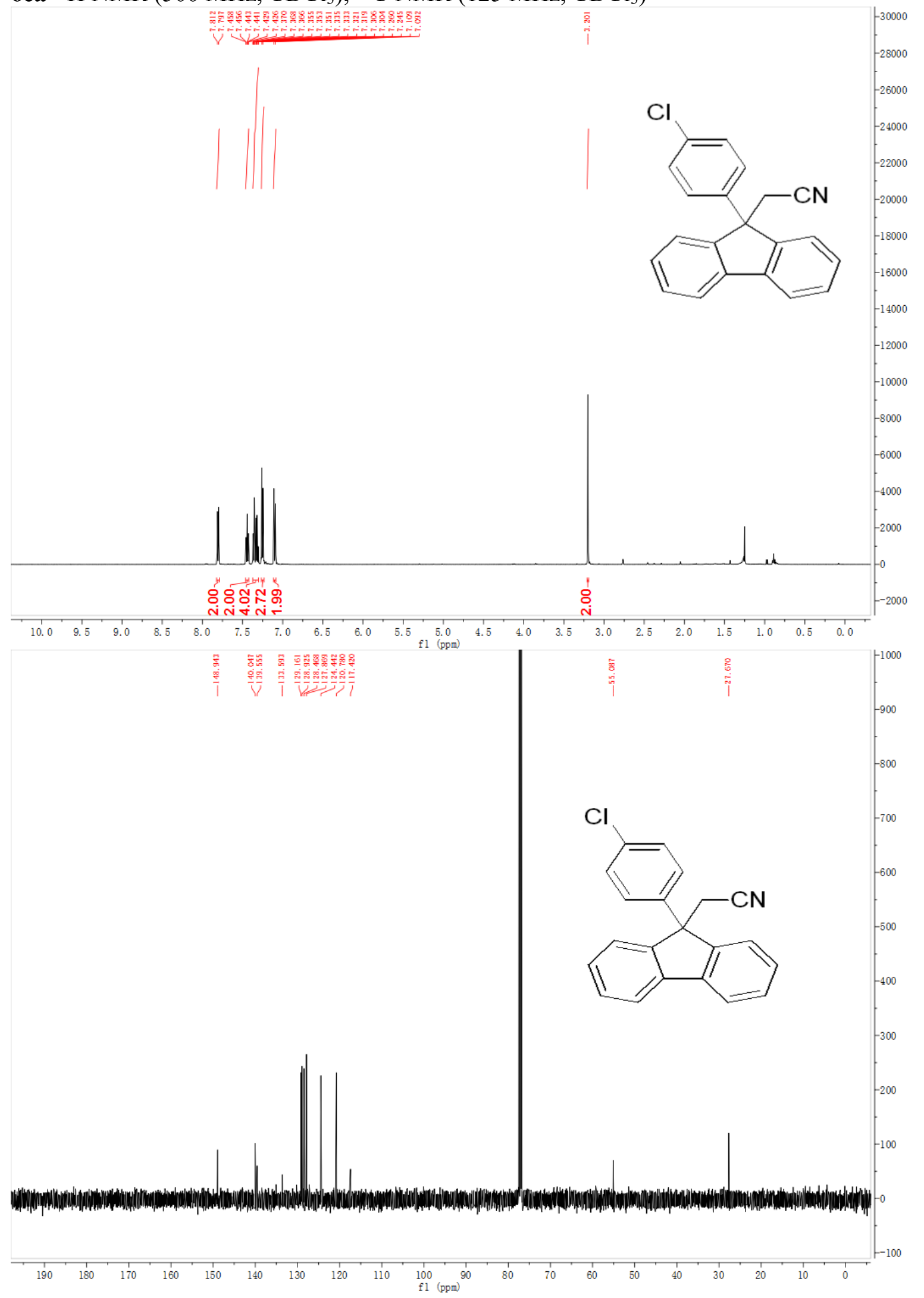


6fa ${ }^{1} \mathrm{H} \mathrm{NMR}\left(500 \mathrm{MHz}, \mathrm{CDCl}_{3}\right),{ }^{13} \mathrm{C} \mathrm{NMR}\left(125 \mathrm{MHz}, \mathrm{CDCl}_{3}\right),{ }^{19} \mathrm{~F} \mathrm{NMR}\left(376 \mathrm{MHz}, \mathrm{CDCl}_{3}\right)$

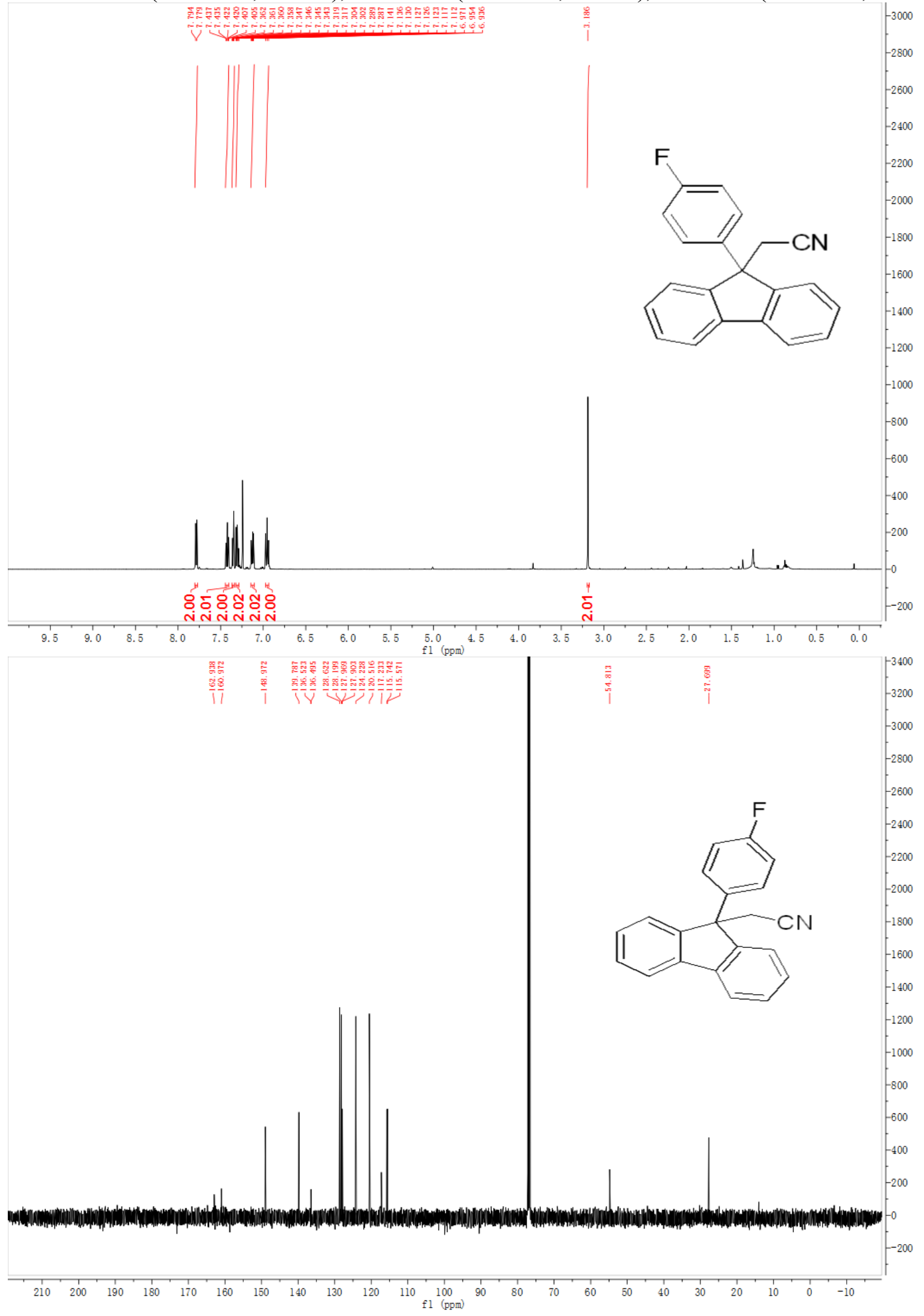




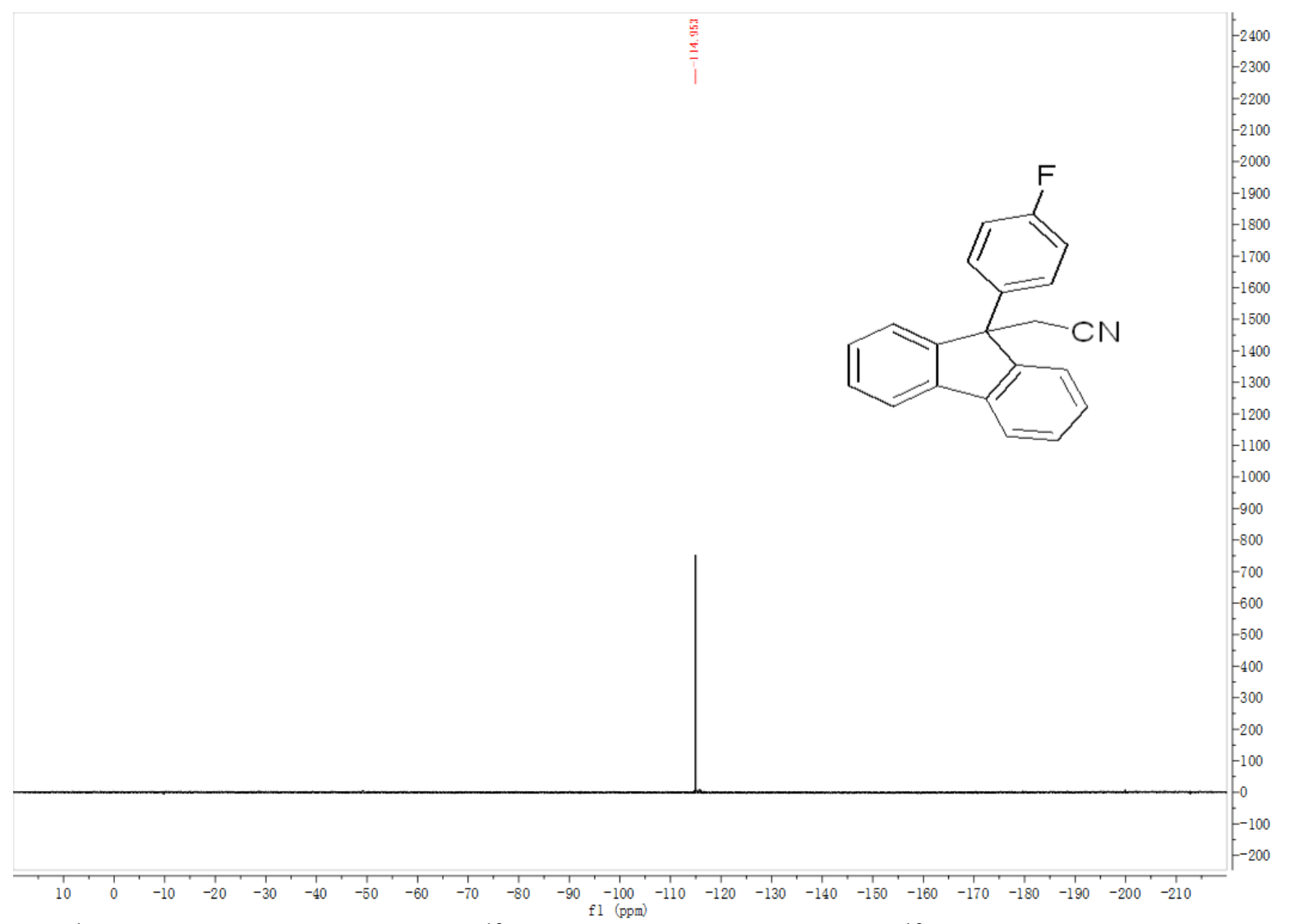

6ga ${ }^{1} \mathrm{H}$ NMR (500 MHz, CDCl $),{ }^{13} \mathrm{C} \mathrm{NMR}\left(125 \mathrm{MHz}, \mathrm{CDCl}_{3}\right),{ }^{19} \mathrm{~F}$ NMR $\left(376 \mathrm{MHz}, \mathrm{CDCl}_{3}\right)$

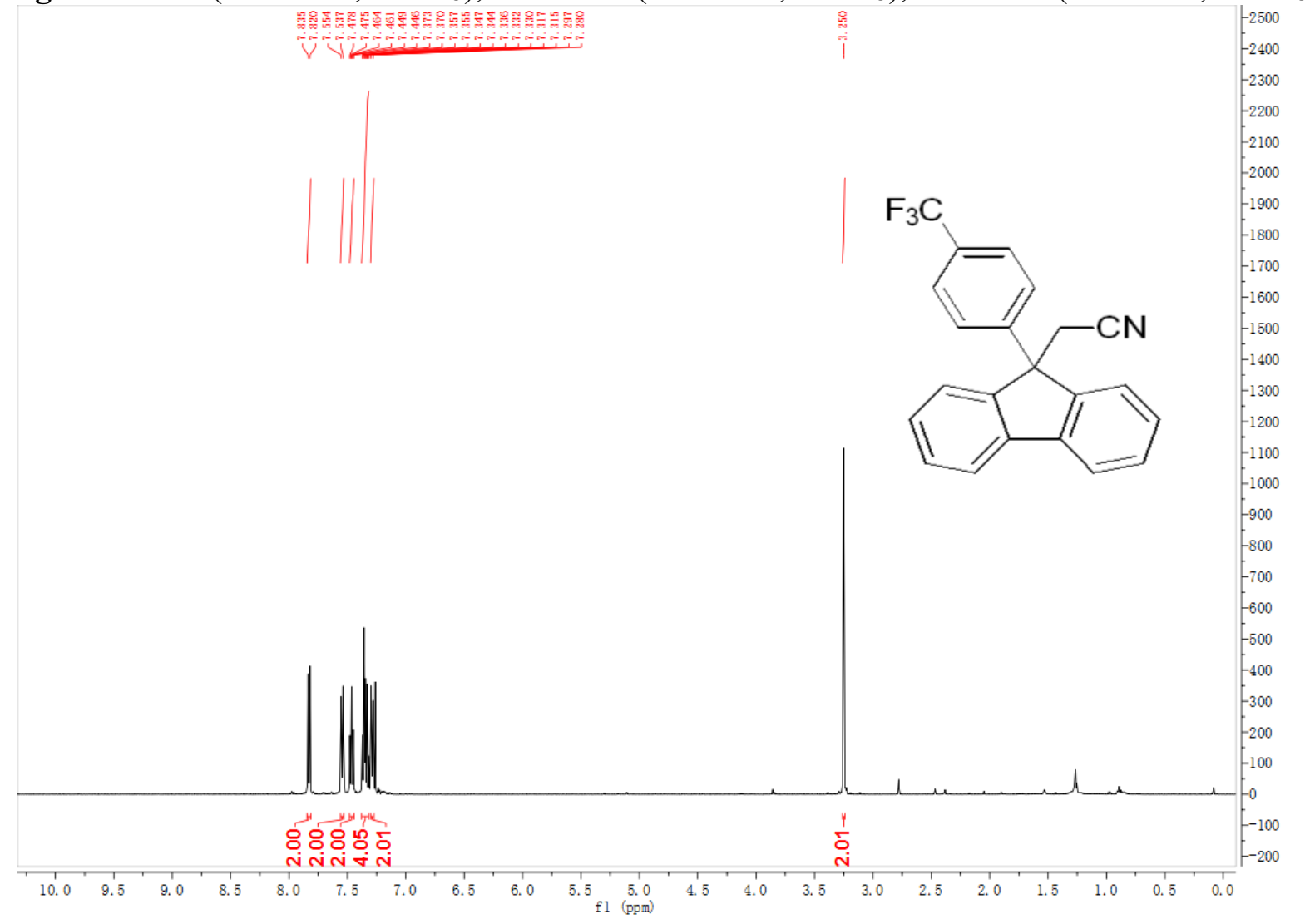




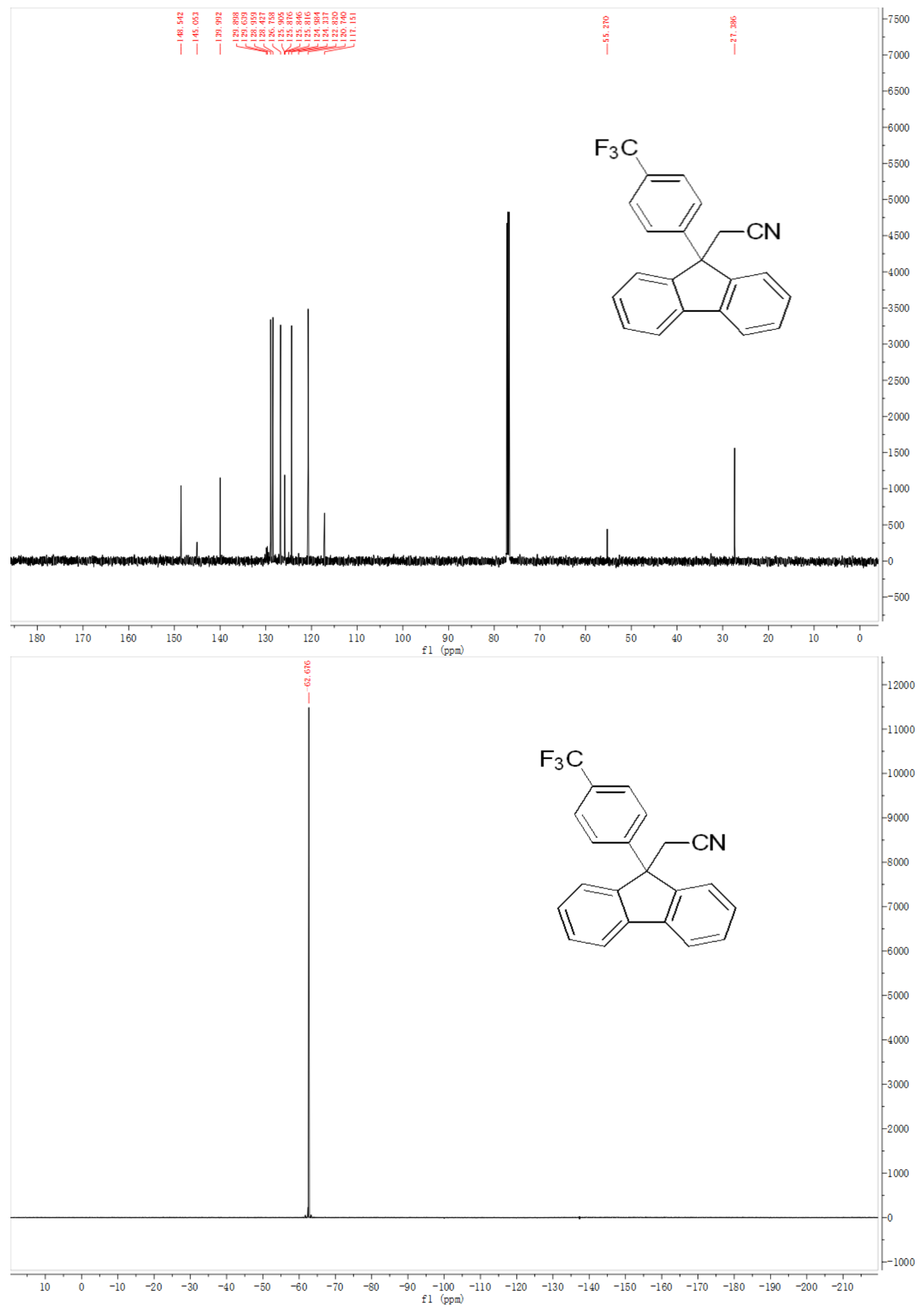


6ha ${ }^{1} \mathrm{H}$ NMR $\left(500 \mathrm{MHz}, \mathrm{CDCl}_{3}\right),{ }^{13} \mathrm{C} \mathrm{NMR}\left(125 \mathrm{MHz}, \mathrm{CDCl}_{3}\right)$

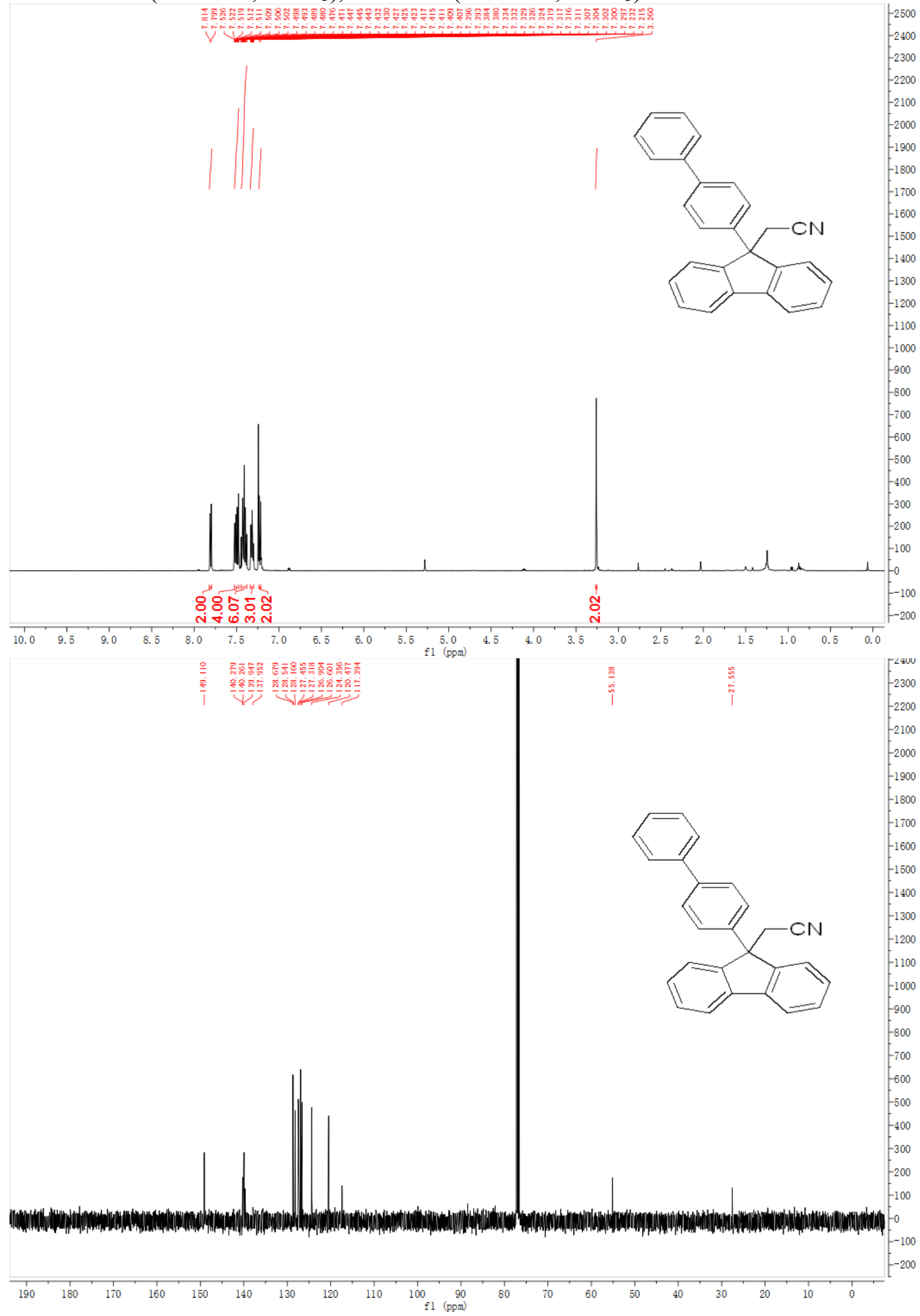


6ia ${ }^{1} \mathrm{H}$ NMR $\left(500 \mathrm{MHz}, \mathrm{CDCl}_{3}\right),{ }^{13} \mathrm{C} \mathrm{NMR}\left(125 \mathrm{MHz}, \mathrm{CDCl}_{3}\right)$
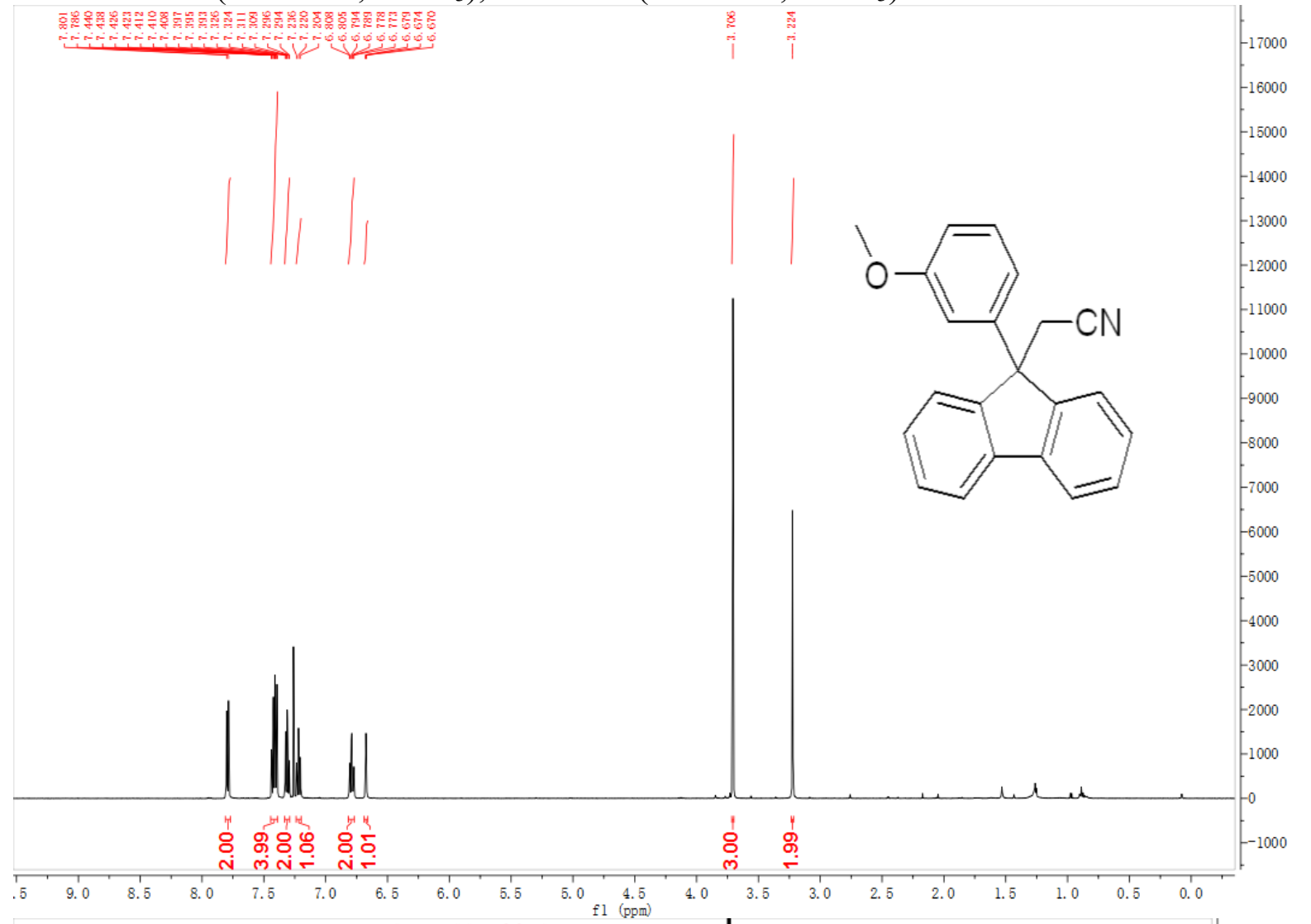

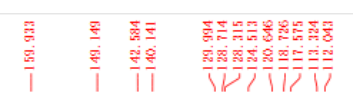

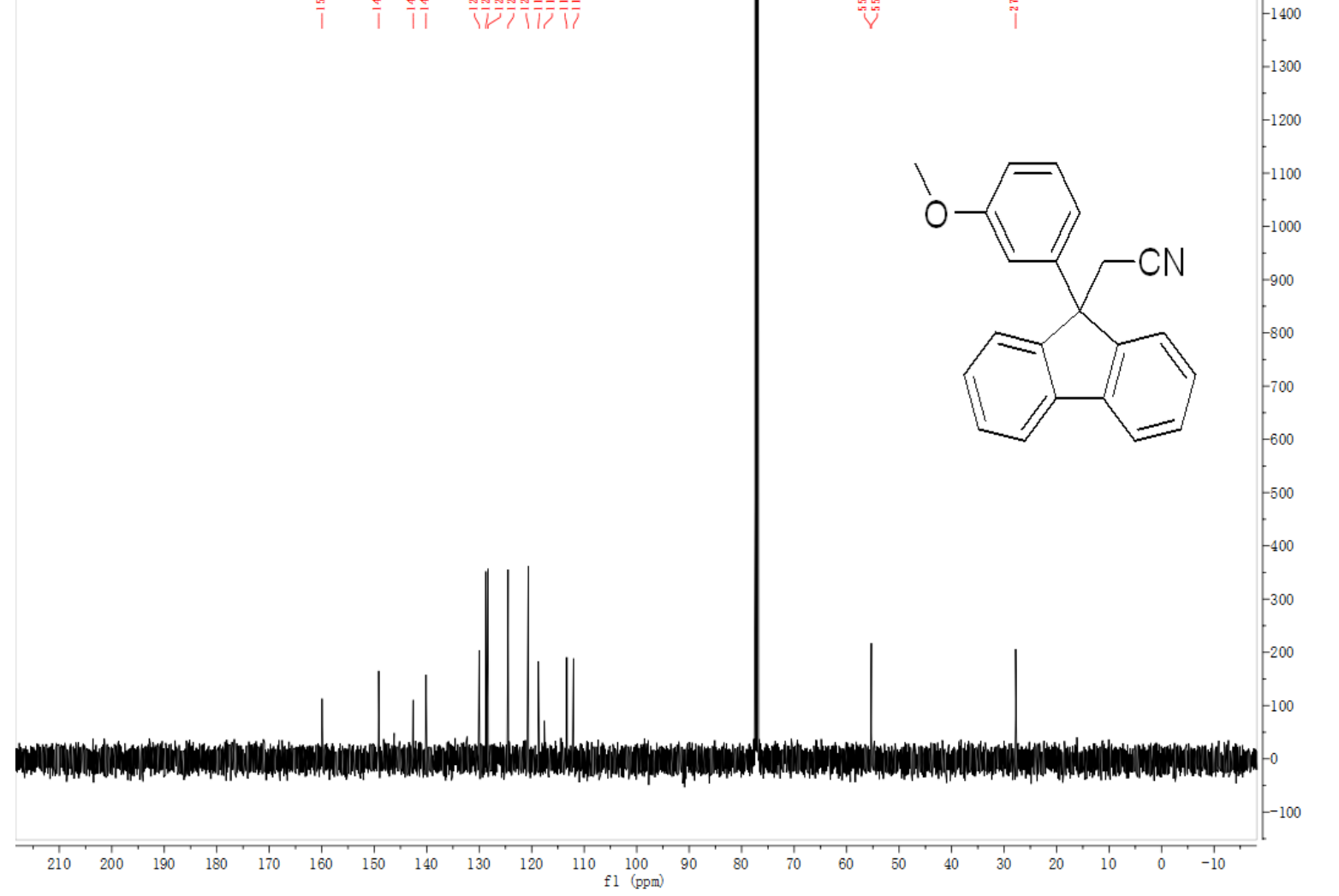


6ja ${ }^{1} \mathrm{H}$ NMR (500 MHz, $\left.\mathrm{CDCl}_{3}\right),{ }^{13} \mathrm{C} \mathrm{NMR}\left(125 \mathrm{MHz}, \mathrm{CDCl}_{3}\right),{ }^{19} \mathrm{~F} \mathrm{NMR}\left(376 \mathrm{MHz}, \mathrm{CDCl}_{3}\right)$

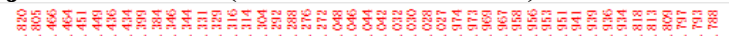

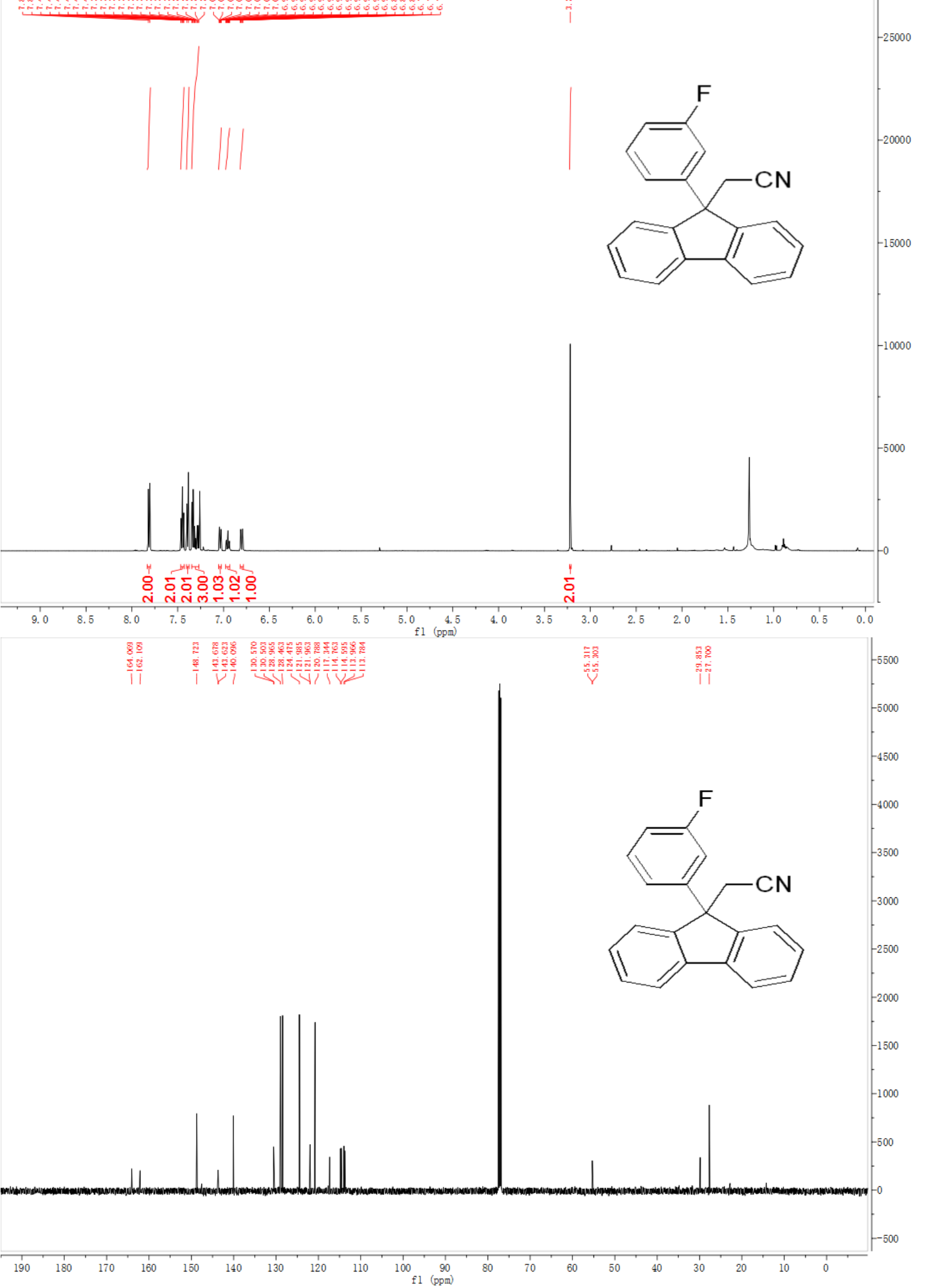




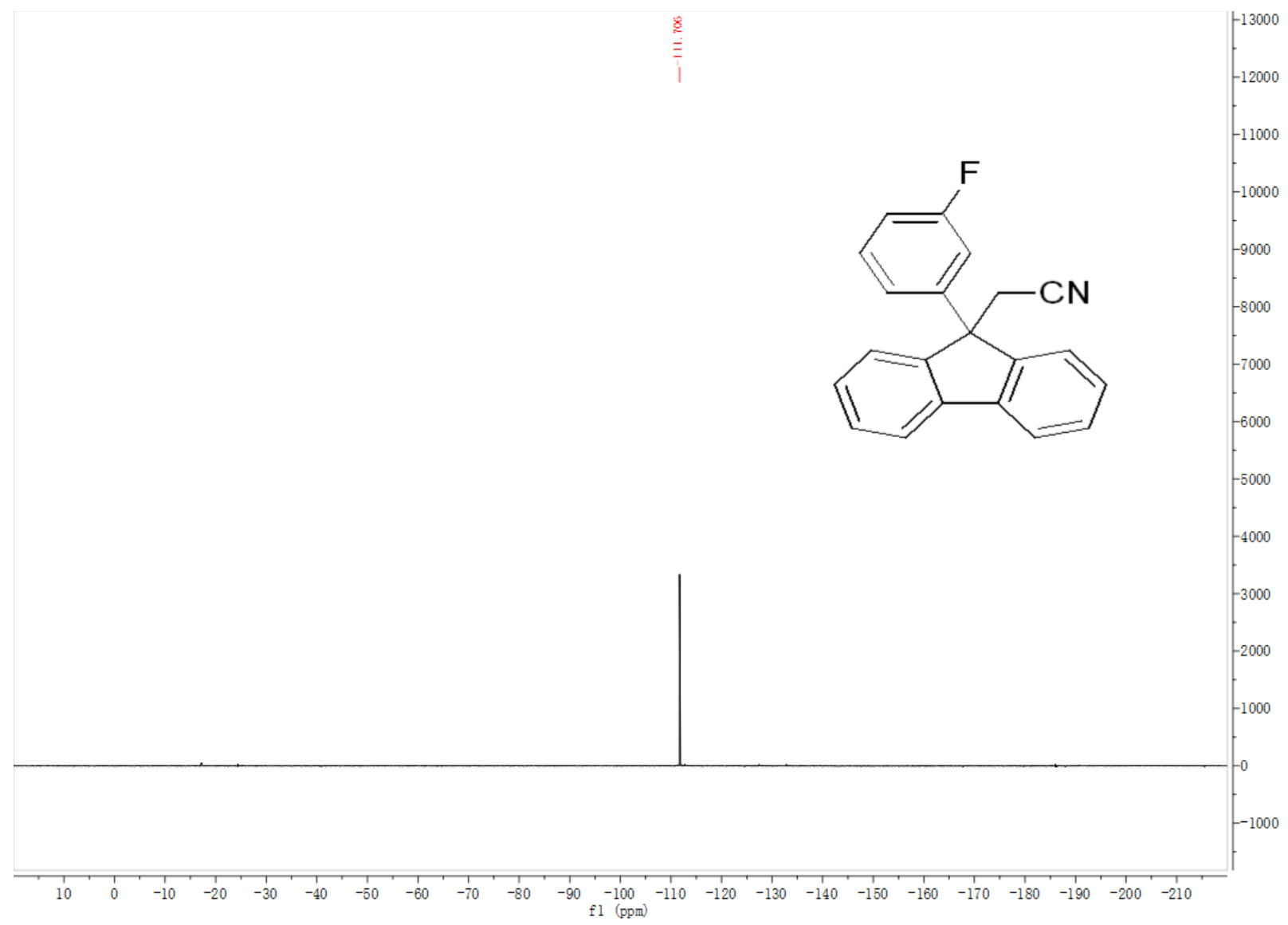


6ka ${ }^{1} \mathrm{H}$ NMR (500 MHz, $\left.\mathrm{CDCl}_{3}\right),{ }^{13} \mathrm{C} \mathrm{NMR}\left(125 \mathrm{MHz}, \mathrm{CDCl}_{3}\right)$

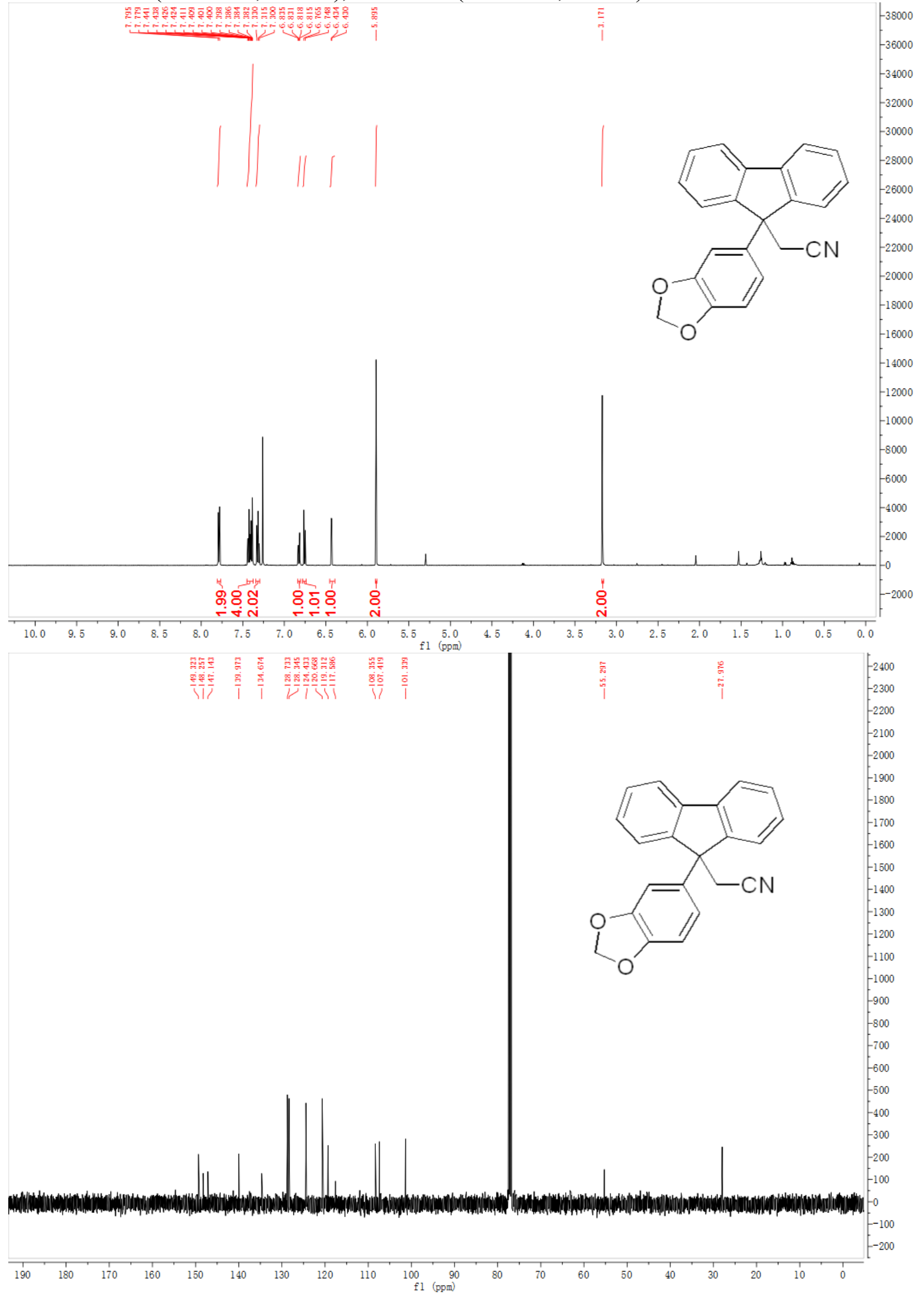


6la ${ }^{1} \mathrm{H} \mathrm{NMR}\left(500 \mathrm{MHz}, \mathrm{CDCl}_{3}\right),{ }^{13} \mathrm{C} \mathrm{NMR}\left(125 \mathrm{MHz}, \mathrm{CDCl}_{3}\right)$

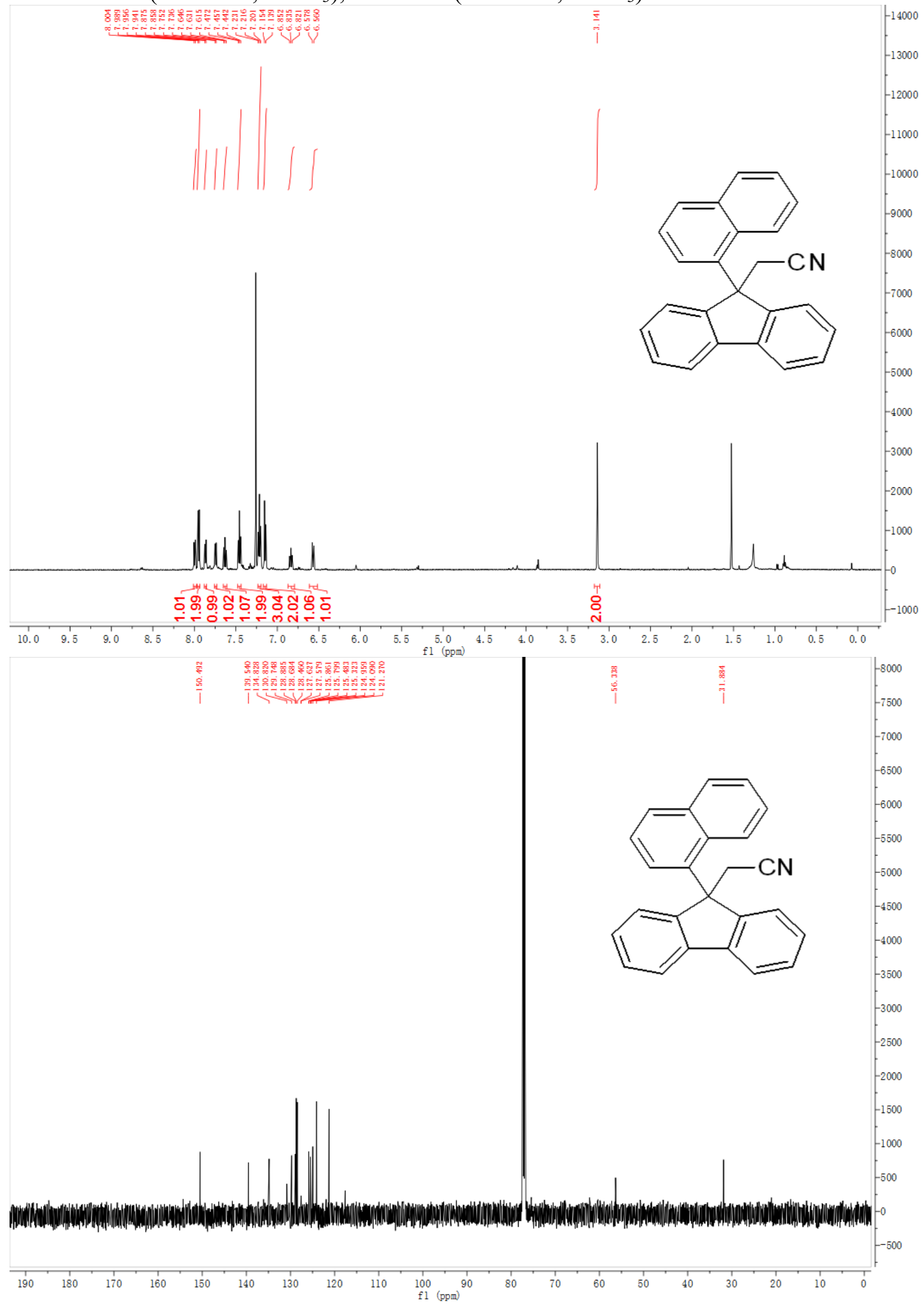


6ma ${ }^{1} \mathrm{H} \mathrm{NMR}\left(500 \mathrm{MHz}, \mathrm{CDCl}_{3}\right),{ }^{13} \mathrm{C} \mathrm{NMR}\left(125 \mathrm{MHz}, \mathrm{CDCl}_{3}\right)$

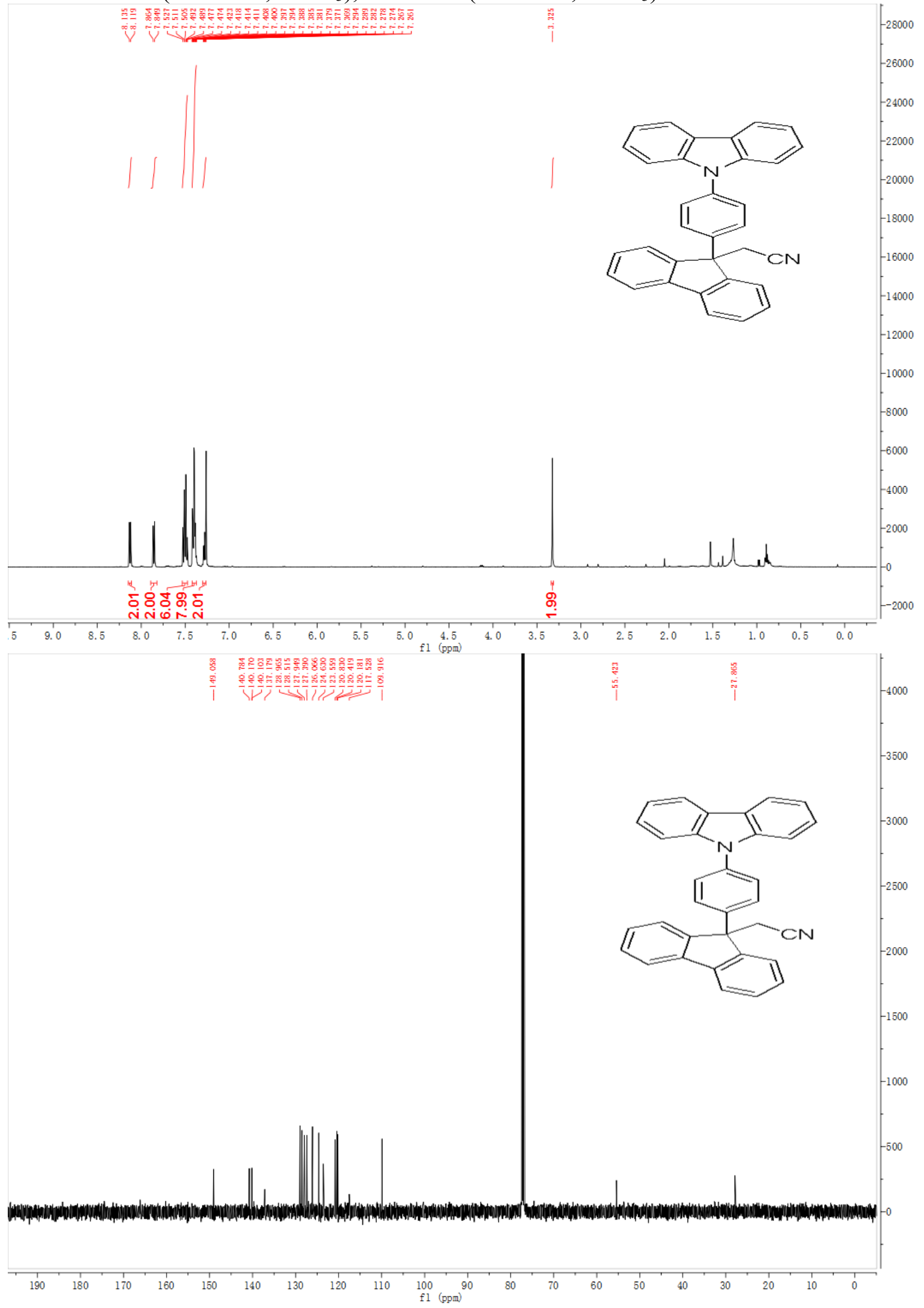




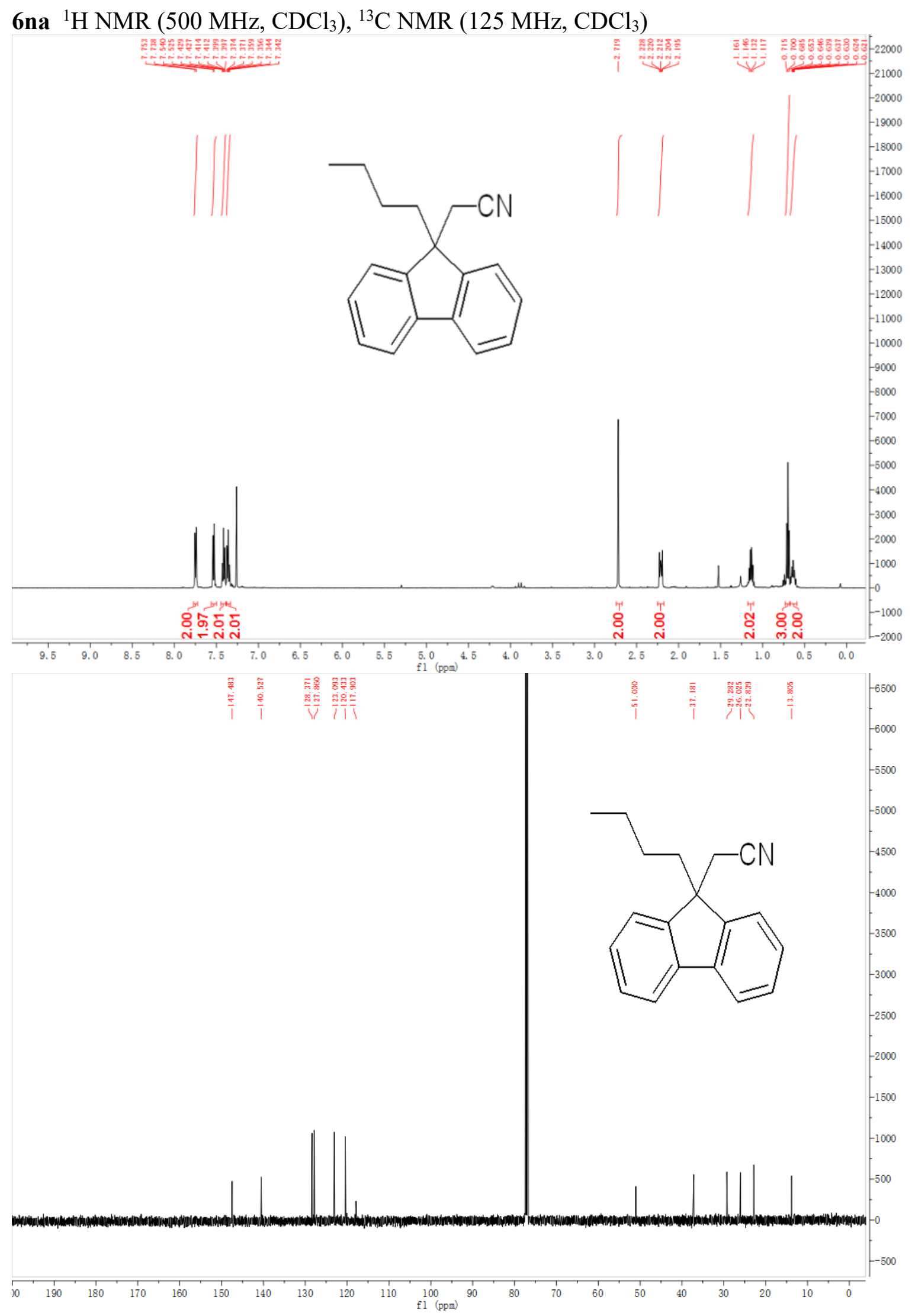


6oa ${ }^{1} \mathrm{H} \mathrm{NMR}\left(500 \mathrm{MHz}, \mathrm{CDCl}_{3}\right),{ }^{13} \mathrm{C} \mathrm{NMR}\left(125 \mathrm{MHz}, \mathrm{CDCl}_{3}\right),{ }^{19} \mathrm{~F} \mathrm{NMR}\left(376 \mathrm{MHz}, \mathrm{CDCl}_{3}\right)$

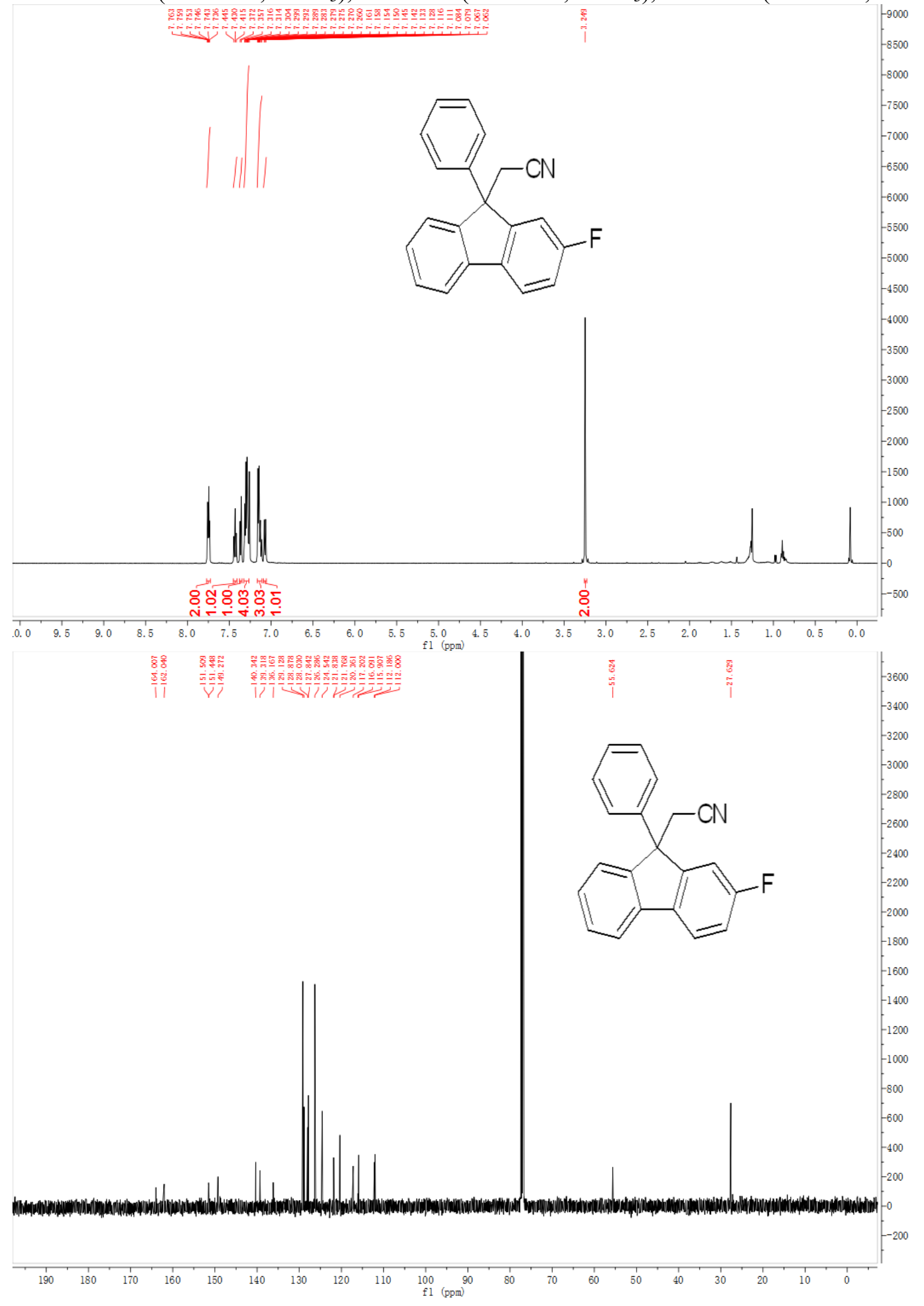




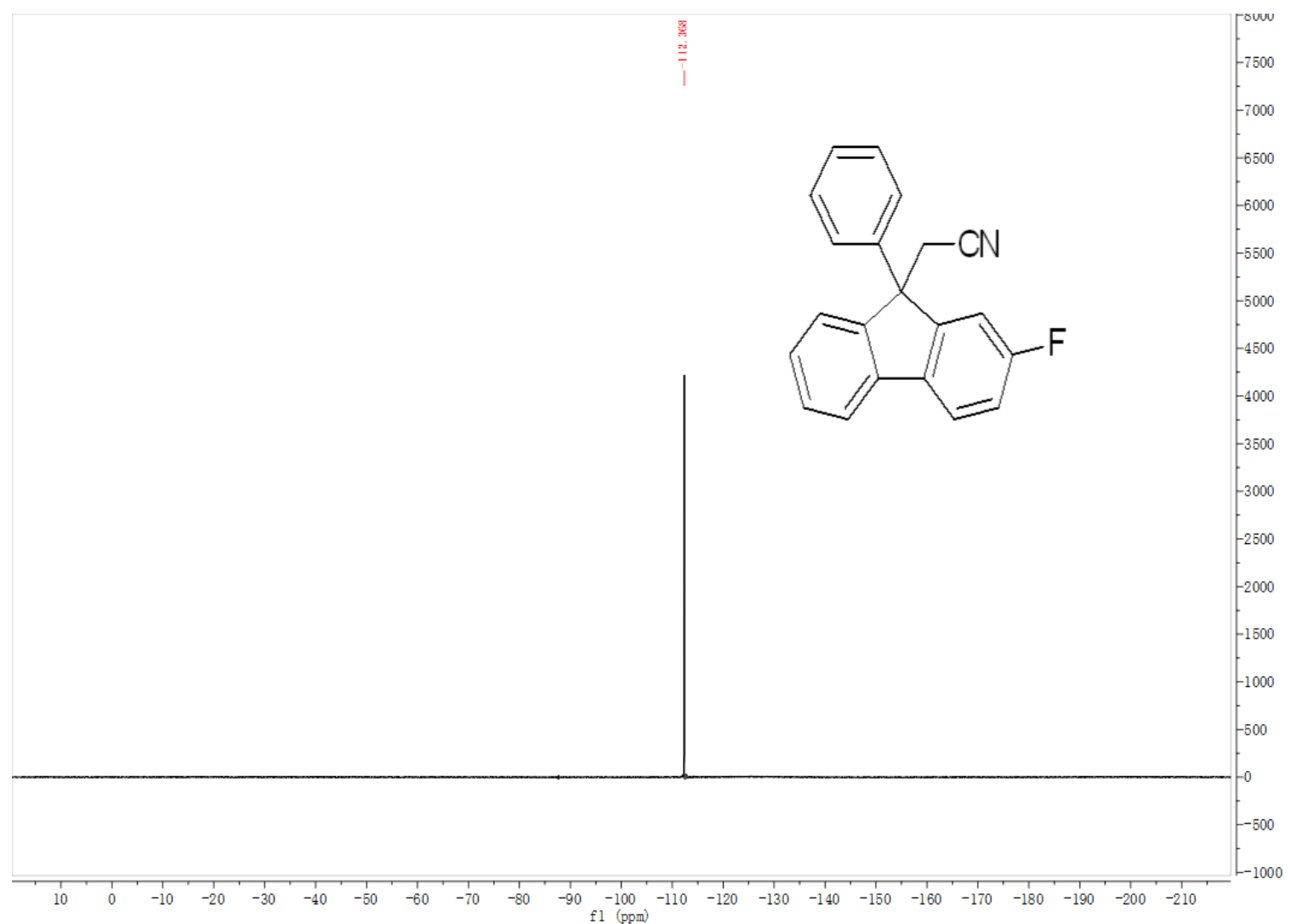




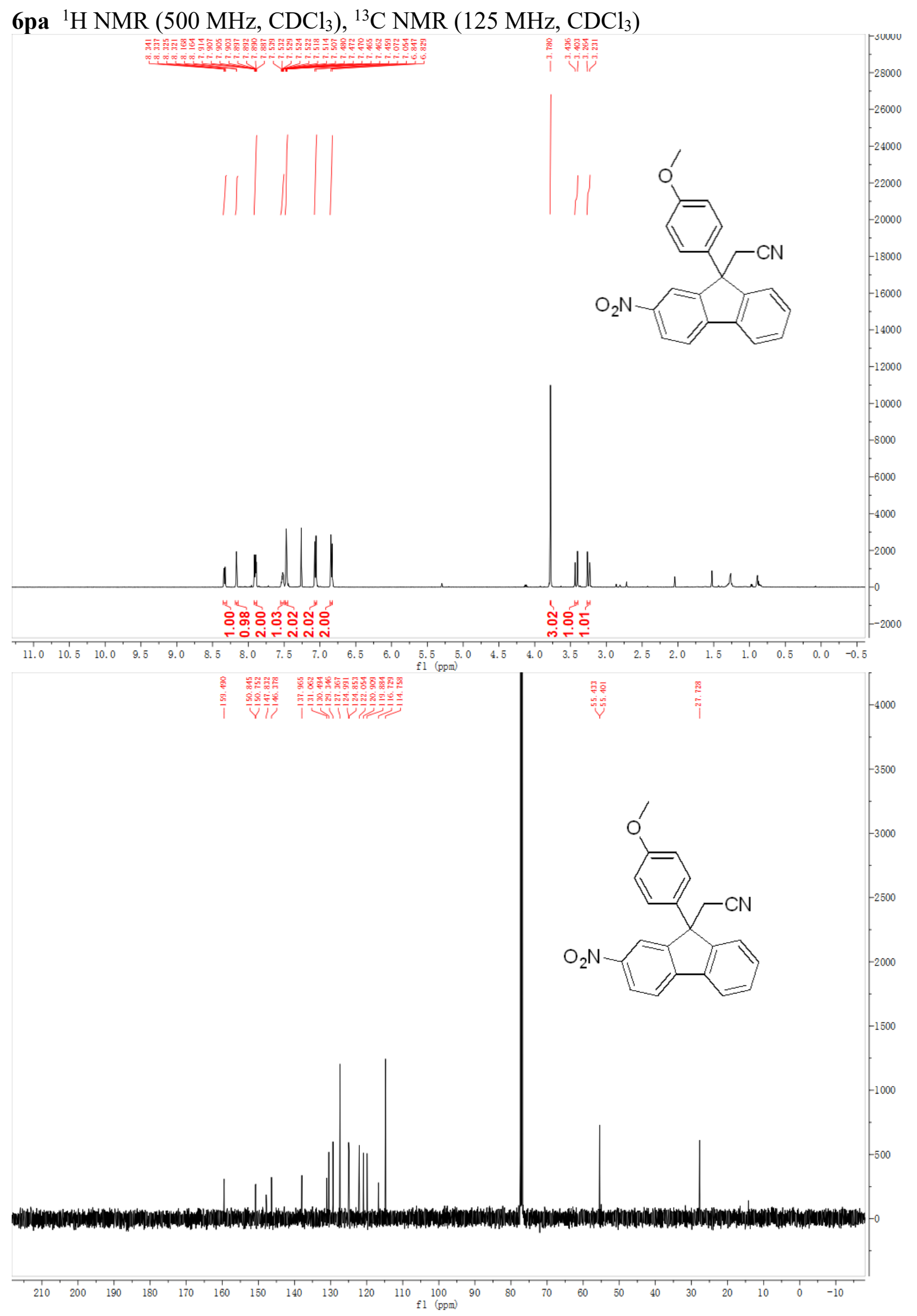


6qa ${ }^{1} \mathrm{H}$ NMR $\left(500 \mathrm{MHz}, \mathrm{CDCl}_{3}\right),{ }^{13} \mathrm{C} \mathrm{NMR}\left(125 \mathrm{MHz}, \mathrm{CDCl}_{3}\right)$

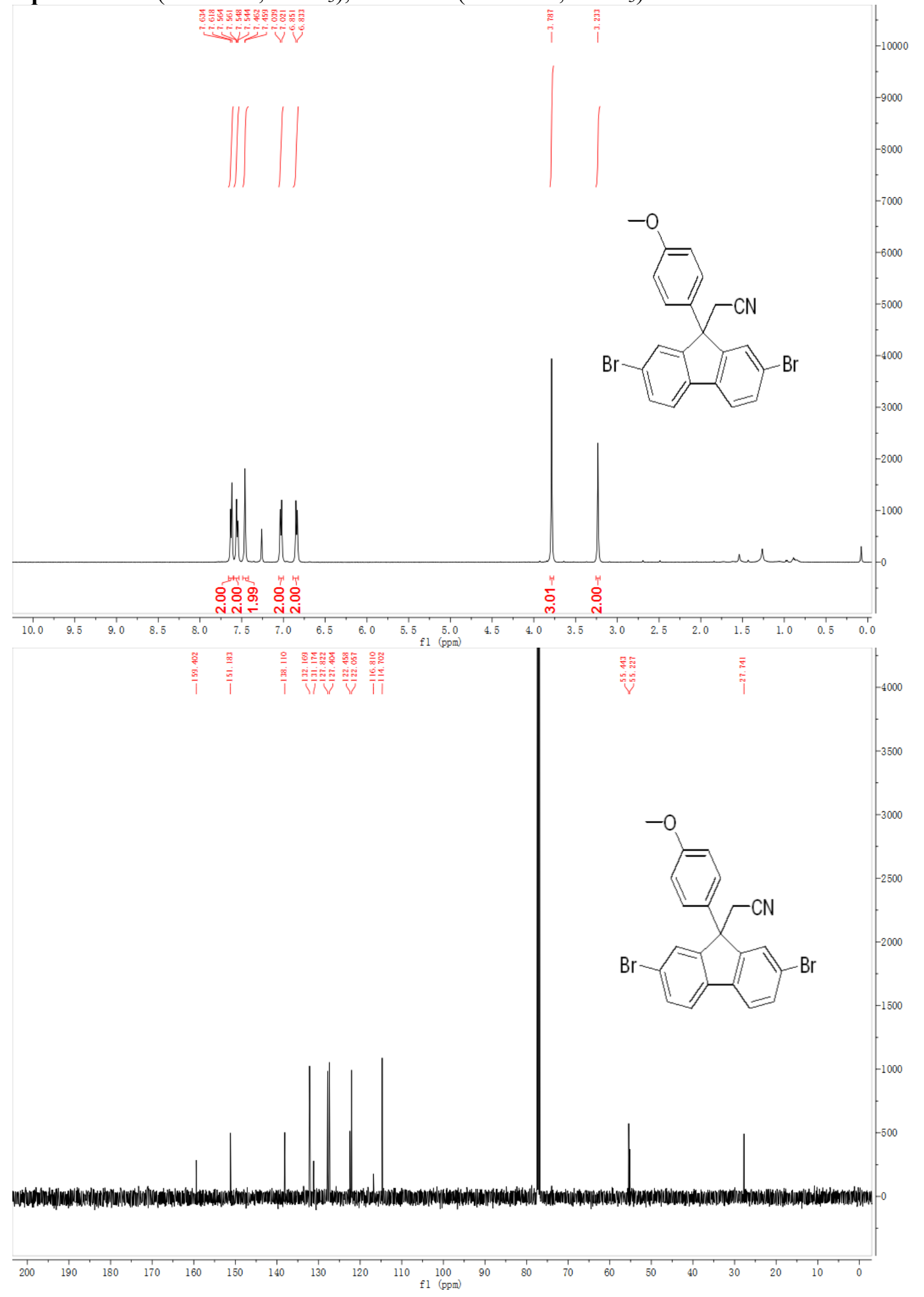




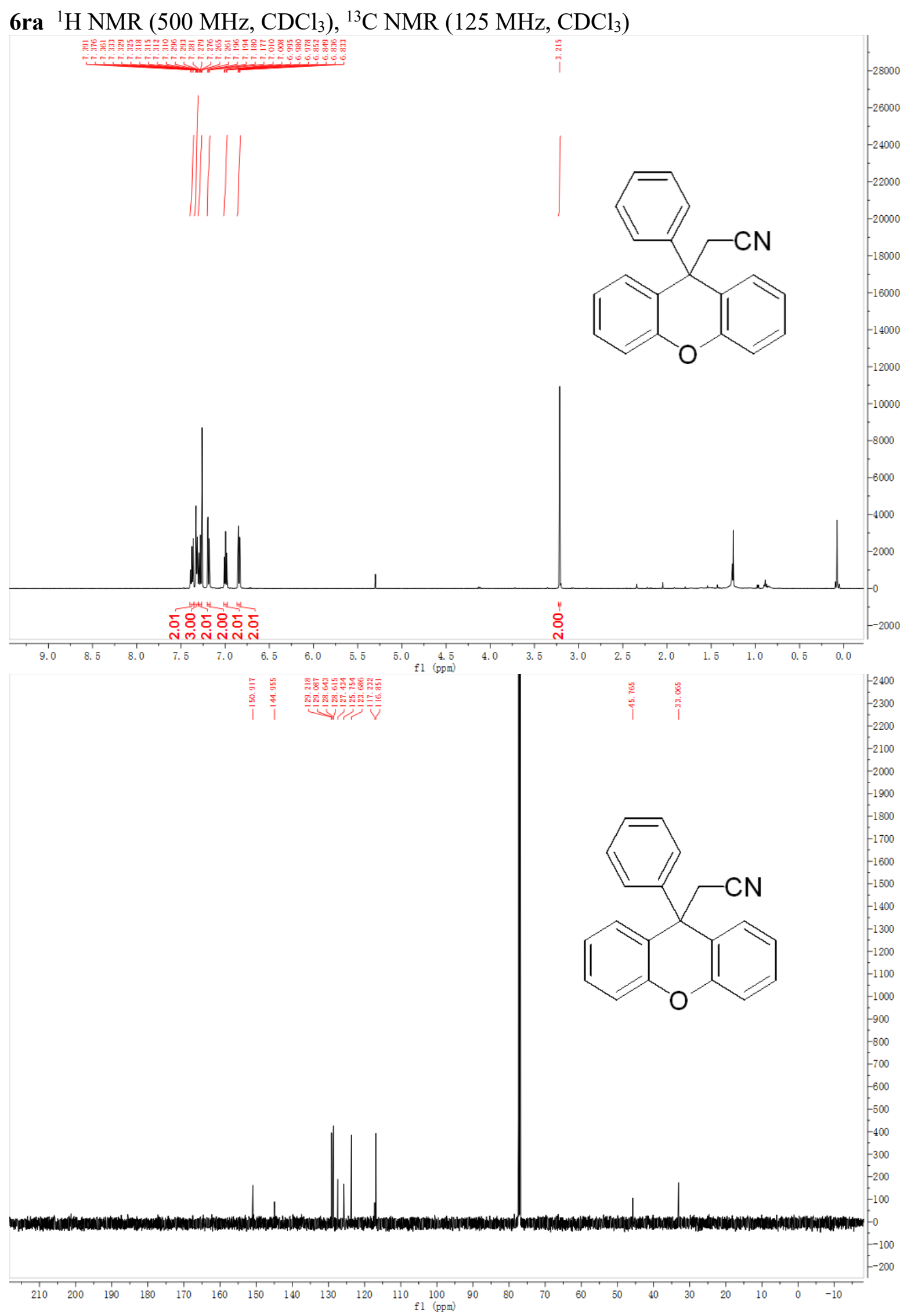


6ab ${ }^{1} \mathrm{H}$ NMR (500 MHz, $\left.\left.\mathrm{CDCl}_{3}\right),{ }^{13} \mathrm{C} \mathrm{NMR} \mathrm{(125} \mathrm{MHz,} \mathrm{CDCl}_{3}\right)$

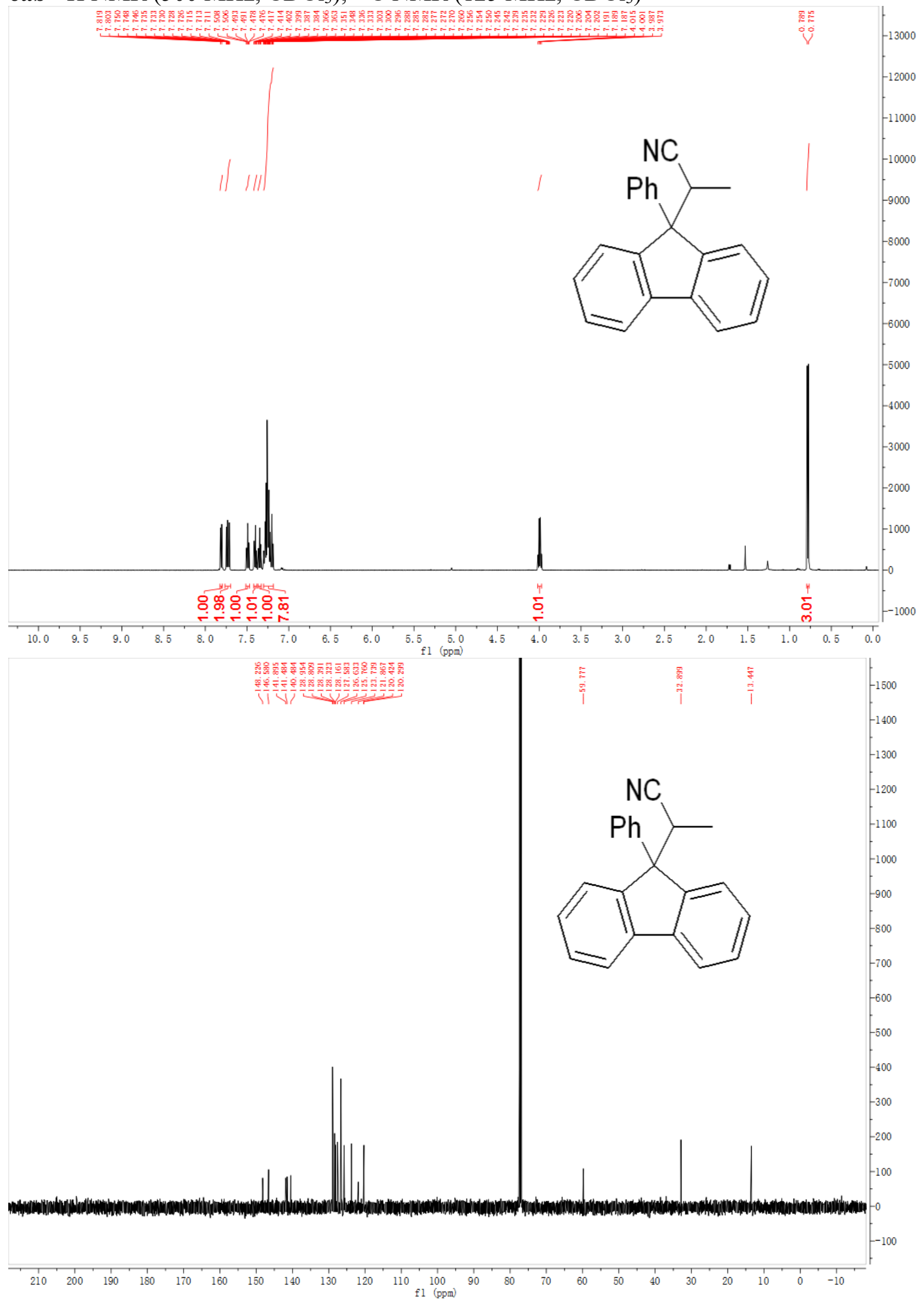




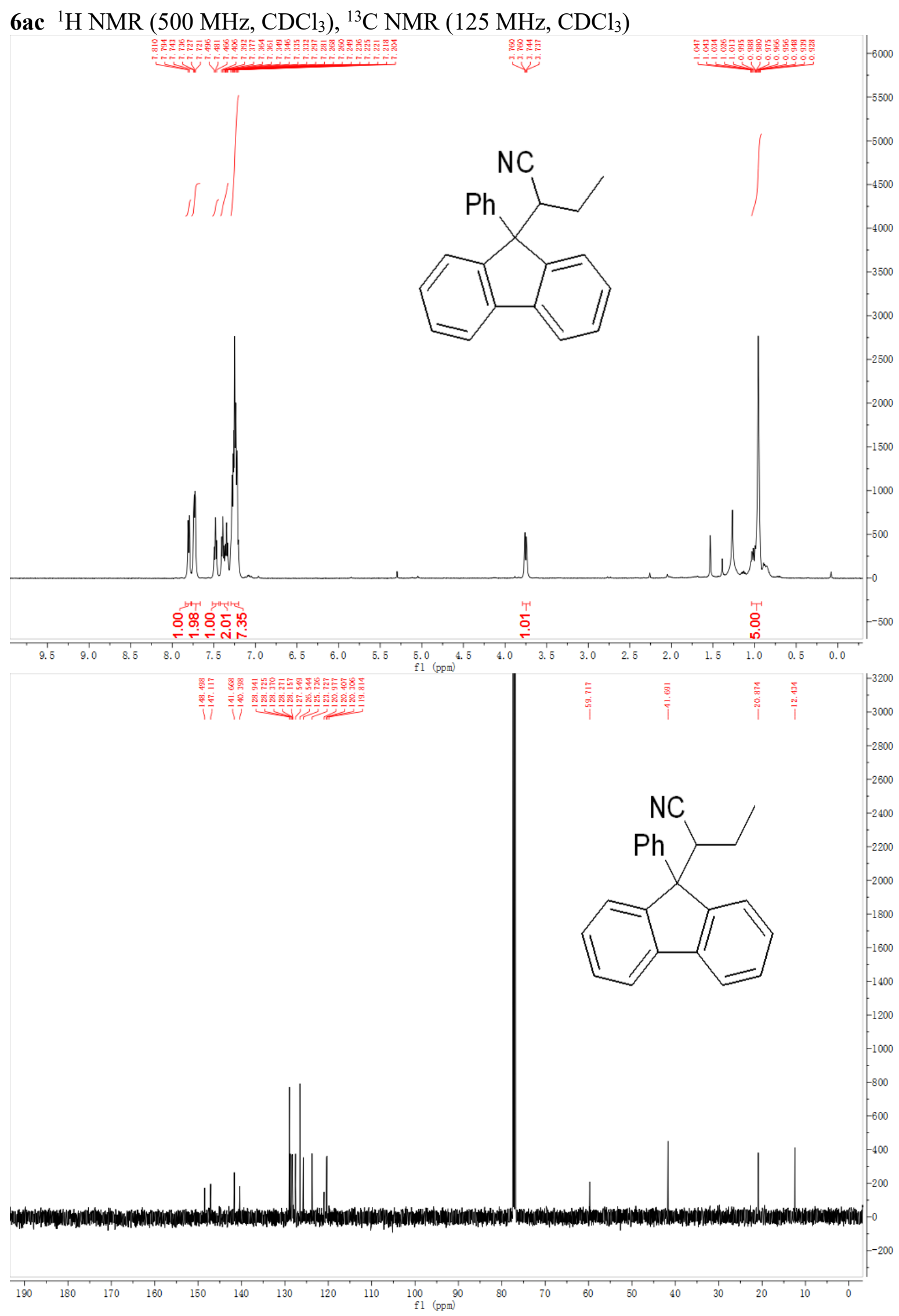


6ad ${ }^{1} \mathrm{H}$ NMR $\left(500 \mathrm{MHz}, \mathrm{CDCl}_{3}\right),{ }^{13} \mathrm{C} \mathrm{NMR}\left(125 \mathrm{MHz}, \mathrm{CDCl}_{3}\right)$

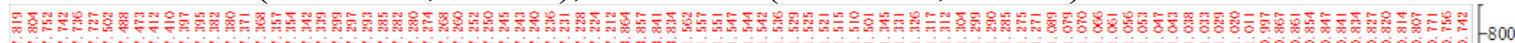

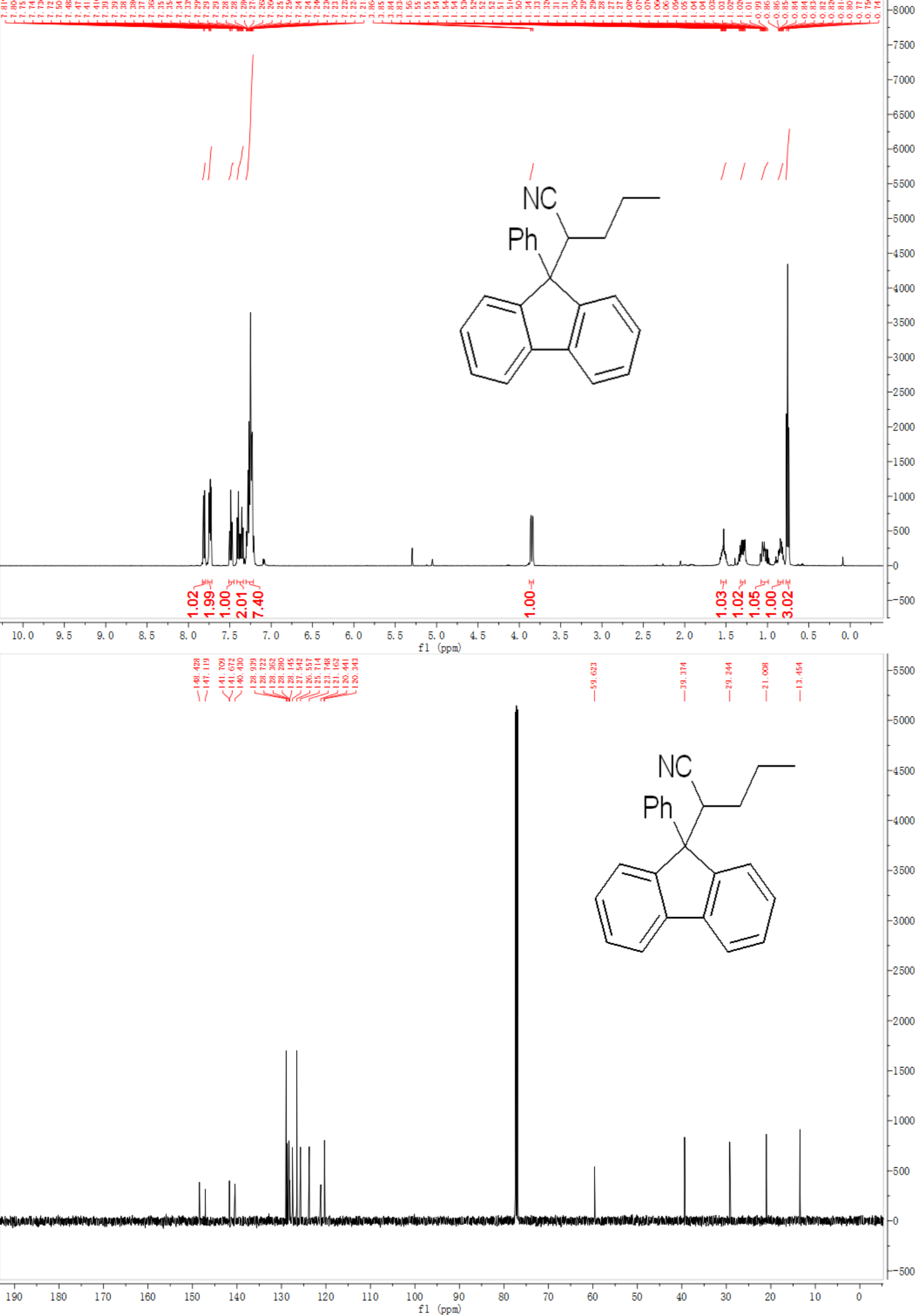


6ae ${ }^{1} \mathrm{H}$ NMR (500 MHz, $\left.\mathrm{CDCl}_{3}\right),{ }^{13} \mathrm{C} \mathrm{NMR}\left(125 \mathrm{MHz}, \mathrm{CDCl}_{3}\right)$

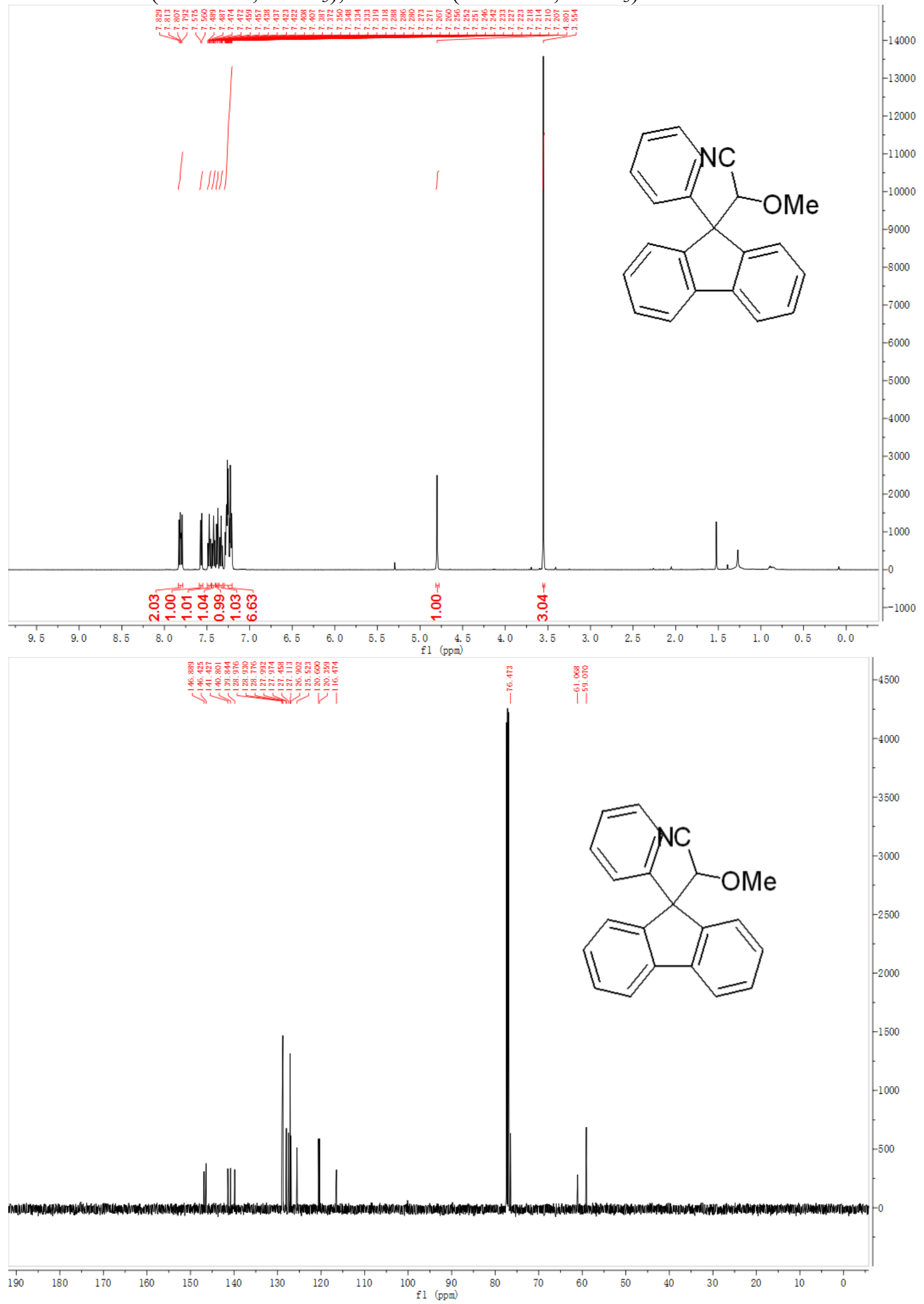


6af ${ }^{1} \mathrm{H} \mathrm{NMR}\left(500 \mathrm{MHz}, \mathrm{CDCl}_{3}\right),{ }^{13} \mathrm{C} \mathrm{NMR}\left(125 \mathrm{MHz}, \mathrm{CDCl}_{3}\right)$

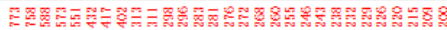

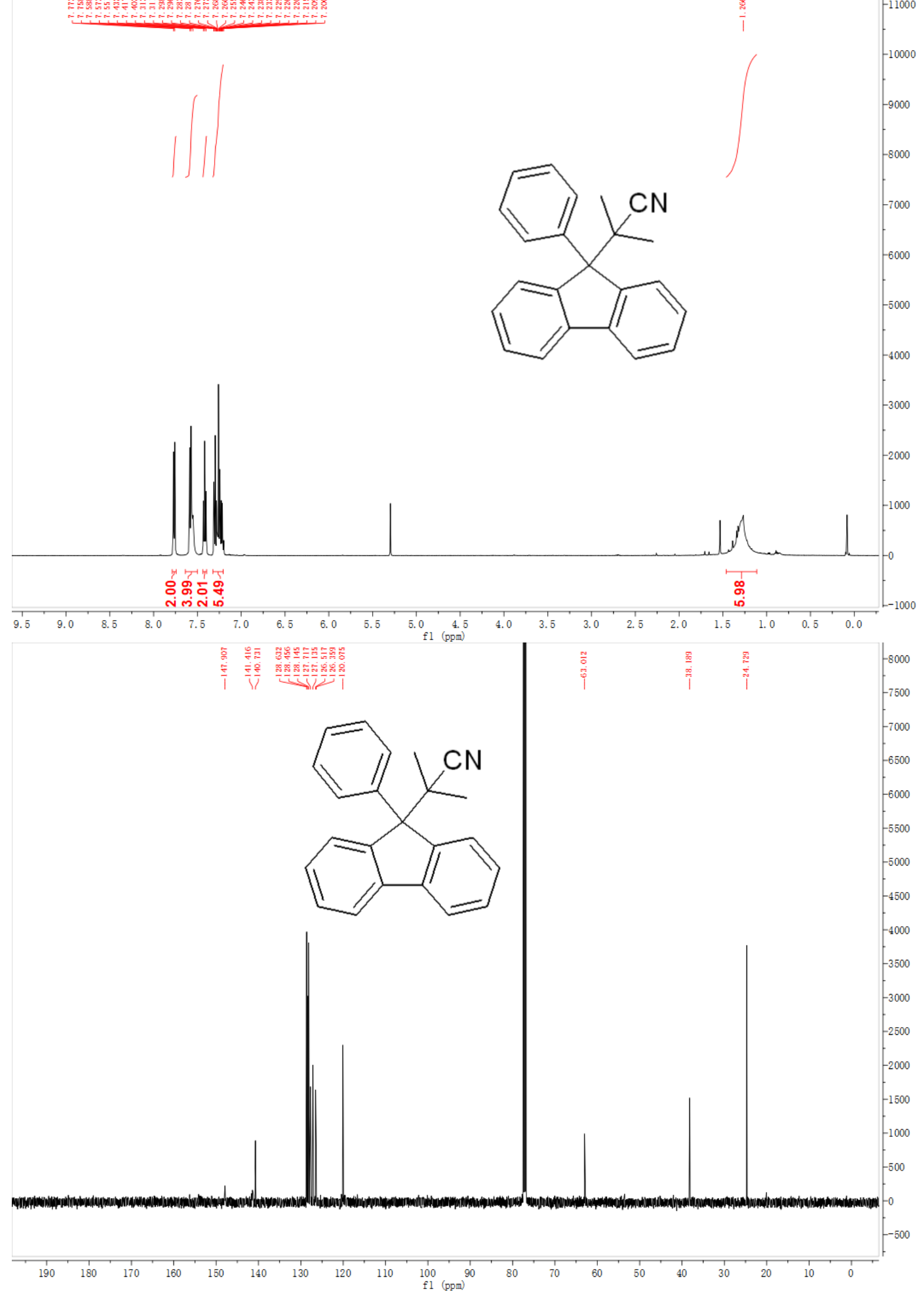


6ag ${ }^{1} \mathrm{H}$ NMR (500 MHz, $\left.\mathrm{CDCl}_{3}\right),{ }^{13} \mathrm{C} \mathrm{NMR}\left(125 \mathrm{MHz}, \mathrm{CDCl}_{3}\right)$

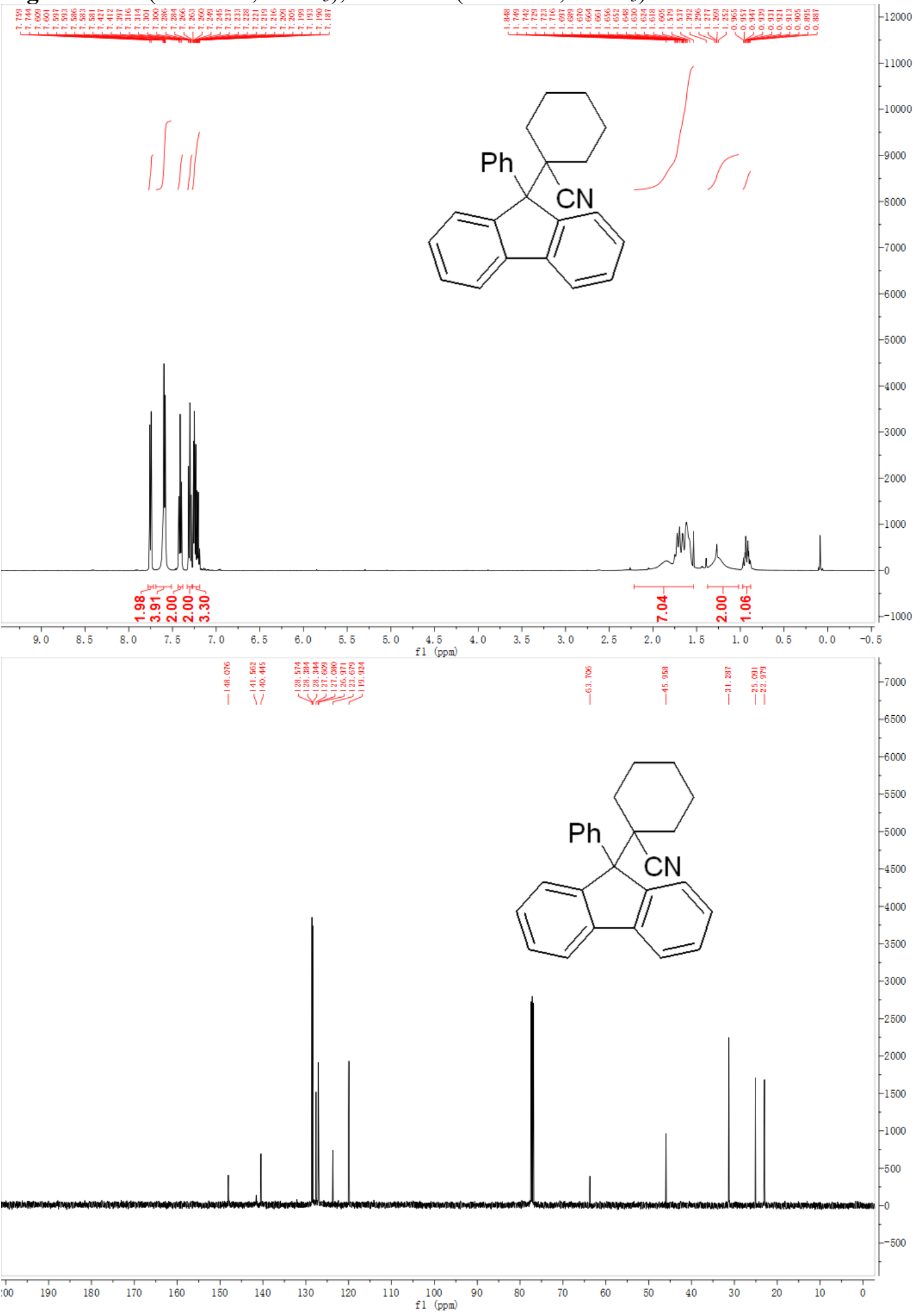


$4{ }^{1} \mathrm{H}$ NMR (500 MHz, $\left.\mathrm{CDCl}_{3}\right),{ }^{13} \mathrm{C} \mathrm{NMR}\left(125 \mathrm{MHz}, \mathrm{CDCl}_{3}\right)$

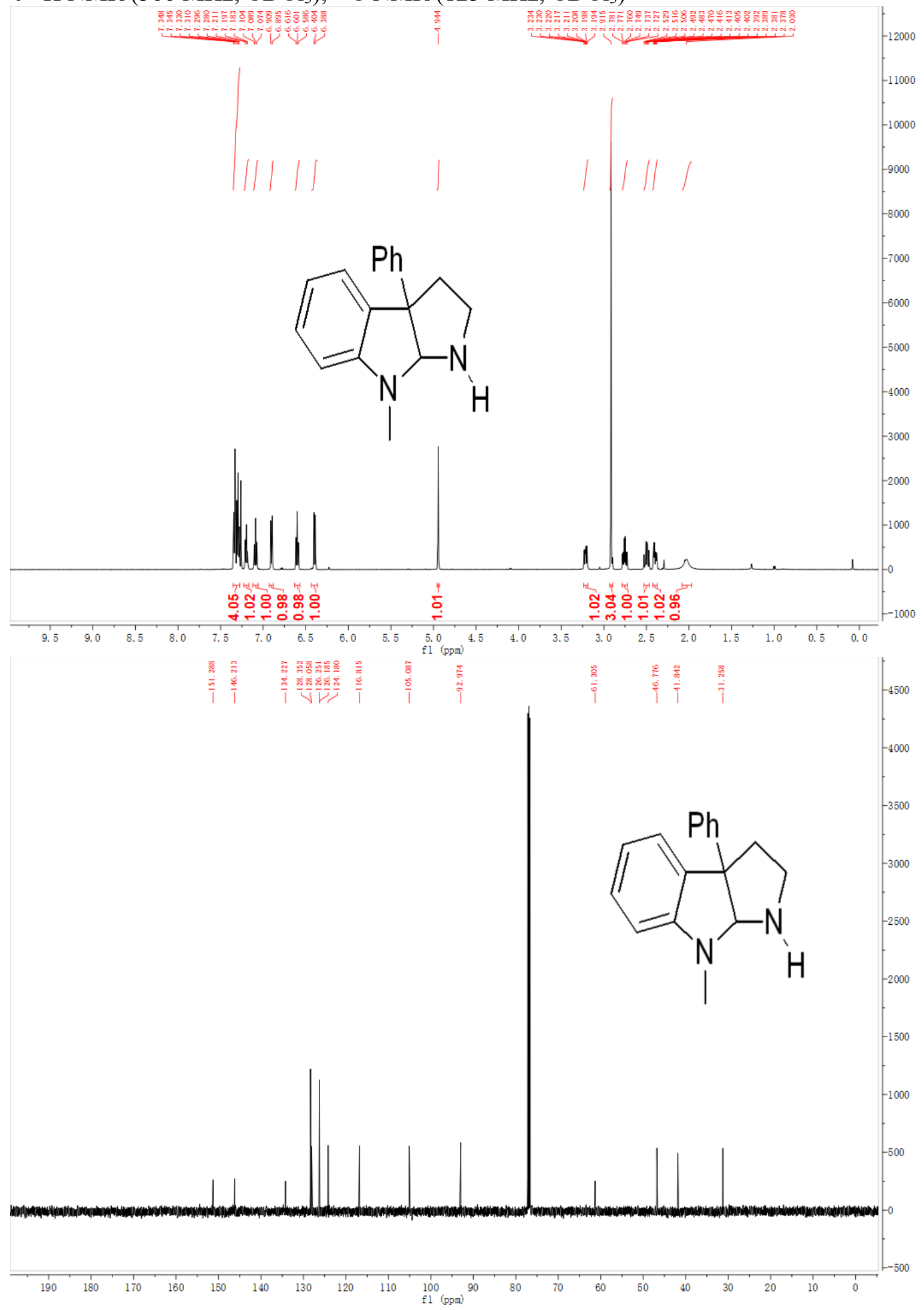


$10{ }^{1} \mathrm{H}$ NMR (500 MHz, $\left.\mathrm{CDCl}_{3}\right)$

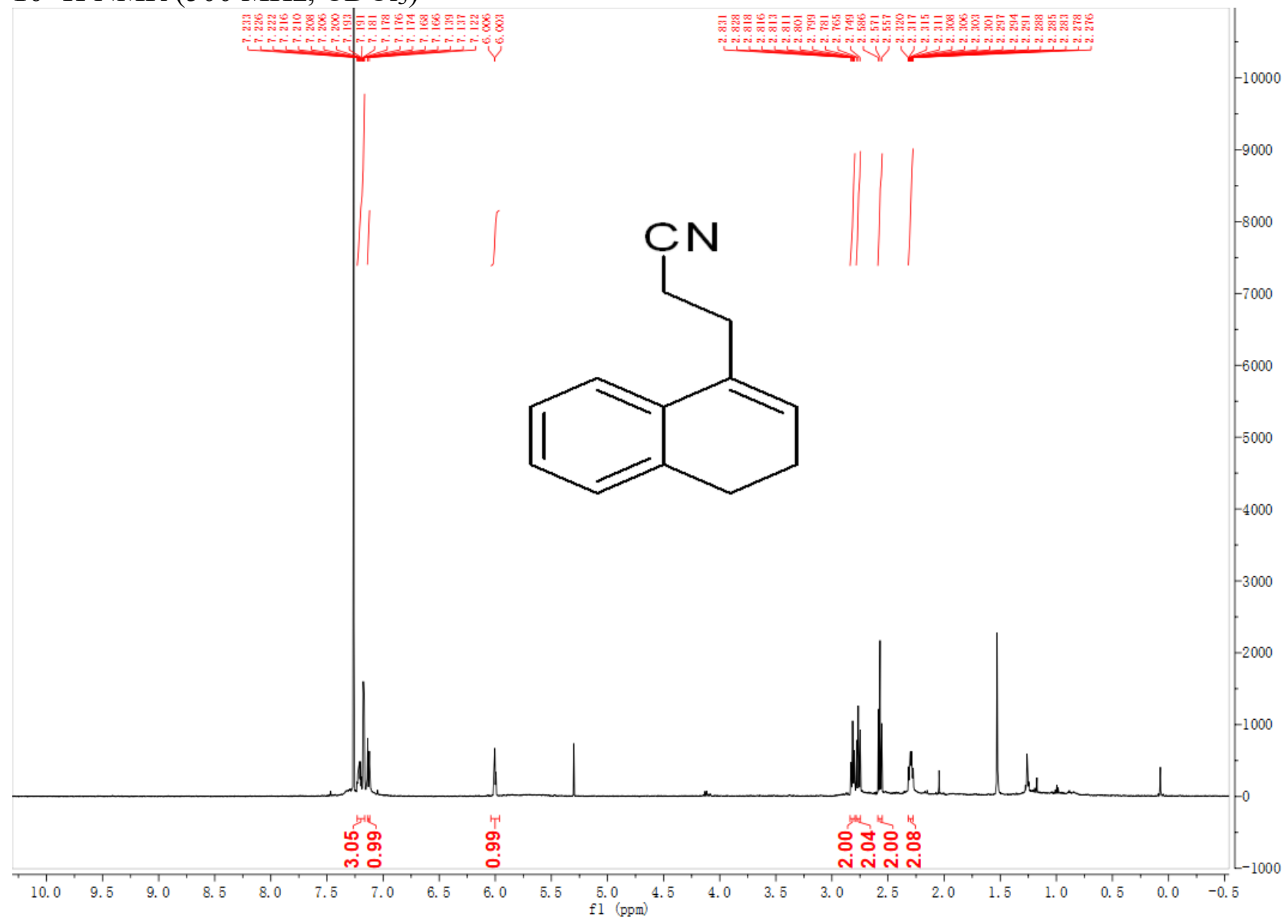

